\title{
Mirror channel eigenvectors of the $d$-dimensional fishnets
}

\author{
Sergey Derkachov, ${ }^{a, b}$ Gwenaël Ferrando $^{c, d}$ and Enrico Olivucci ${ }^{e}$ \\ ${ }^{a}$ St.Petersburg Department of the Steklov Mathematical Institute of Russian Academy of Sciences, \\ Fontanka 27, 191023, St.Petersburg, Russia \\ ${ }^{b}$ National Research University Higher School of Economics, \\ Soyuza Pechatnikov, 16, St.Petersburg, 190008, Russia \\ ${ }^{c}$ Laboratoire de Physique de l'École Normale Supérieure, CNRS, Université PSL, \\ Sorbonne Université, \\ 24 rue Lhomond, 75005 Paris, France \\ ${ }^{d}$ Université Paris-Saclay, CNRS, CEA, Institut de Physique Théorique, \\ 91191 Gif-sur-Yvette, France \\ ${ }^{e}$ Perimeter Institute for Theoretical Physics, \\ Waterloo, Ontario N2L2Y5, Canada \\ E-mail: derkach@pdmi.ras.ru, gwenael@tauex.tau.ac.il, \\ eolivucci@perimeterinstitute.ca
}

ABSTRACT: We present a basis of eigenvectors for the graph building operators acting along the mirror channel of planar fishnet Feynman integrals in $d$-dimensions. The eigenvectors of a fishnet lattice of length $N$ depend on a set of $N$ quantum numbers $\left(u_{k}, l_{k}\right)$, each associated with the rapidity and bound-state index of a lattice excitation. Each excitation is a particle in $(1+1)$-dimensions with $O(d)$ internal symmetry, and the wave-functions are formally constructed with a set of creation/annihilation operators that satisfy the corresponding Zamolodchikovs-Faddeev algebra. These properties are proved via the representation, new to our knowledge, of the matrix elements of the fused R-matrix with $O(d)$ symmetry as integral operators on the functions of two spacetime points. The spectral decomposition of a fishnet integral we achieved can be applied to the computation of Basso-Dixon integrals in higher dimensions.

KEywords: Conformal Field Theory, Field Theories in Higher Dimensions, Lattice Integrable Models

ArXiv EPrint: 2108.12620 


\section{Contents}

1 Introduction 1

$2 \quad O(d)$-invariant R Matrices $\quad 4$

2.1 Fusion procedure 5

2.2 Spectral decomposition 11

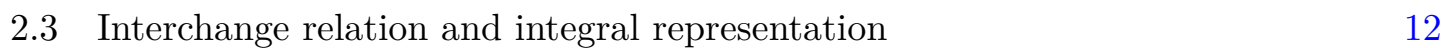

$\begin{array}{lll}2.4 & \text { Properties of the } \mathrm{R} \text { matrices } & 17\end{array}$

3 Diagonalisation of graph-building operators $\quad 23$

3.1 Construction of the eigenvectors 23

$\begin{array}{ll}3.2 & \text { Symmetry property }\end{array}$

3.3 Inner product 29

3.4 Completeness 32

4 Basso-Dixon diagrams $\quad 32$

4.1 Ladder diagrams 34

4.2 Two-layer diagrams 35

5 Conclusions 38

$\begin{array}{ll}\text { A Basic integral relations } & 39\end{array}$

B Equivalence (2.49) and (2.33) 42

B.1 Derivation of (2.50) 42

$\begin{array}{ll}\text { B.2 Equivalence } & 44\end{array}$

$\begin{array}{lr}C \text { Equivalence (2.3) and (2.54) } & 49\end{array}$

$\begin{array}{ll}\text { D Derivative identity } & 51\end{array}$

E Spinor basis

E.1 Star-triangle relation in $d=2 r \quad 55$

\section{Introduction}

The fishnet integrals are a class of Feynman diagrams with square lattice topology [1] of remarkable importance for massless quantum field theory and - especially - for theories with conformal symmetry. Diagrams of fishnet type describe the planar limit of correlators in the strongly-deformed $\mathcal{N}=4$ supersymmetric Yang-Mills theory introduced by V.Kazakov and O.Gürdoğan [2]. Moreover, for minimal size of the square lattice (ladder integrals), they form the basis of functions needed for the bootstrap of four-point functions of $\frac{1}{2}$-BPS operators with specific R-symmetry polarisations in the undeformed theory [3, 4]. 

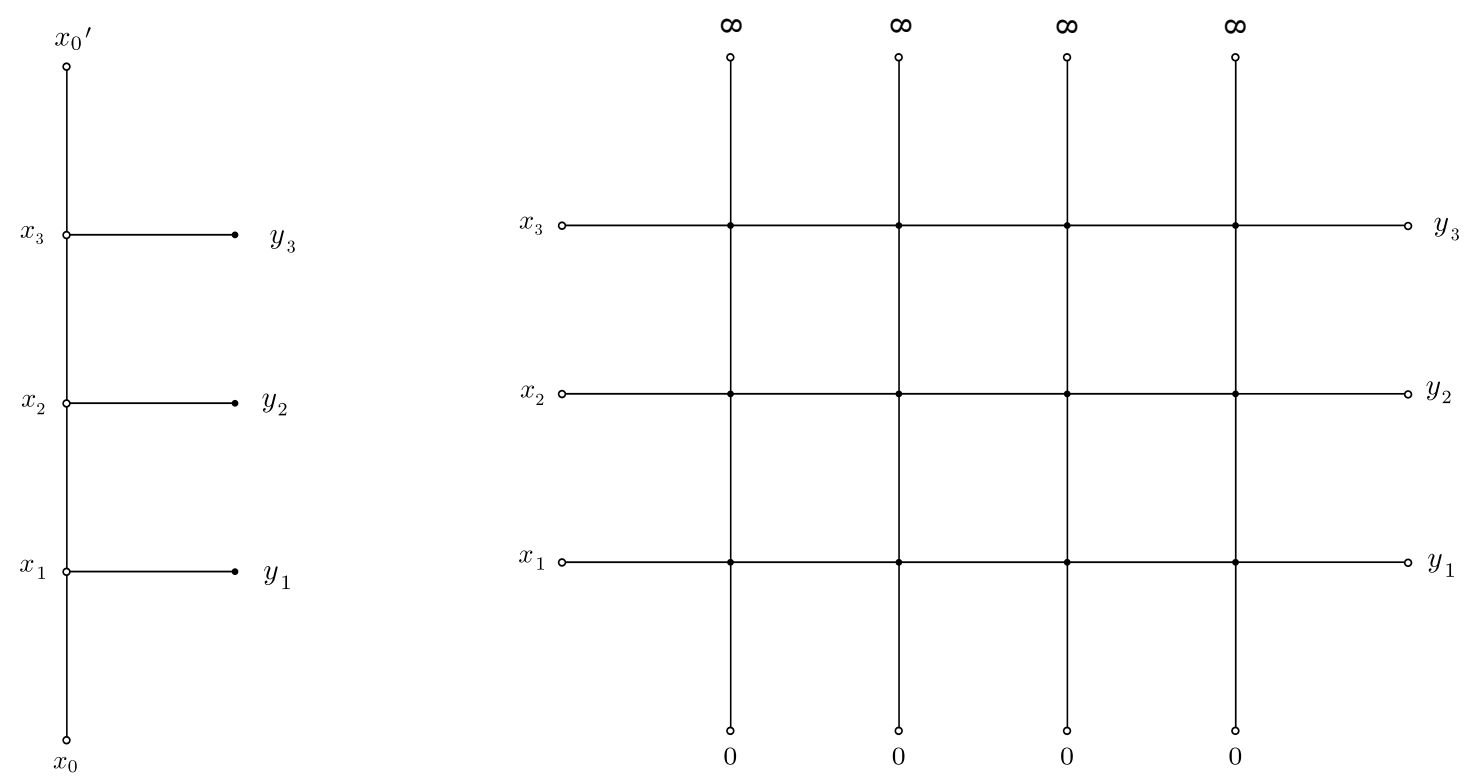

Figure 1. Left: graph-building operator for a fishnet integral of size $N=3$, with fixed boundaries $x_{0}$ and $x_{0}^{\prime}$. Solid lines are bare propagators. Right: fishnet integral of size $M \times N$ with $M=4$ and $N=3$. The boundaries are fixed to points $x_{0}=0$ and $x_{0}^{\prime}=\infty$ (amputation of upper legs), for which the graph-builder is especially simple to diagonalise. The Basso-Dixon integral would correspond to a reduction $x_{k}=x$ and $y_{k}=y$. Black dots are integrated vertices and circles are external points.

Furthermore, other classes of fishnet integrals - with different lattice topology - describe completely the correlators of other planar conformal field theories, for instance the $3 D$ chiral theory obtained as a deformation of ABJM super-conformal theory [5]. Finally, we shall mention that specific fishnets describe the Landau singularity of massless scattering amplitudes at all-loops [6].

The remarkable properties of the fishnets is the possibility to find algorithms for their computation at any loop order [7]. The procedure relies on methods of quantum integrability that map the Feynman integral to (the integral kernel of) a diagonalisable operator the transfer-matrix of an integrable XXX spin chain with conformal symmetry [8, 9]. Also, for a square lattice without boundary conditions imposed, these integrals enjoy infinitedimensional Yangian symmetry [10, 11].

A successful application of the spin-chain tools to fishnets is the computation of BassoDixon (BD) integrals in $d=2,4$ spacetime dimensions. BD integrals [7] are specific reductions of a fishnet with open boundaries, which can be constructed using a so-called graph-building operator, see figure 1. This graph-building operator is said to act in the "mirror channel" of the fishnet lattice. B. Basso and L. Dixon originally obtained [7] a nice explicit determinant formula for this general family of Feynman diagrams in $d=4$. Their derivation may be decomposed in two steps. First, the Feynman diagram is rewritten in some specific representation, then, the obtained expression is transformed to a determinant. The BD integrals in two dimensions were computed in [12], and in that case the transition to the determinant form is straightforward. In $d=4$ the analogue transformation is more complicated and was proven in [13]. 
It was demonstrated in $[12,14,15]$ that the representation obtained in the first step is the so-called separated variables representation. Quantum separation of variables (SoV), introduced by E. Sklyanin [16-18], is one of the various techniques [17, 19-21] used to solve quantum integrable models.

Roughly speaking, SoV consists in finding a basis of the quantum space in which the spectral problem simplifies drastically. Thus, it can be understood as some far-reaching generalisation of the usual Fourier transform. From the point of view of quantum mechanics, the Fourier transform is a transition from coordinate representation to momentum representation. It is the simplest example of a canonical transformation, and the generalised eigenvectors of the momentum operator are used as the integral kernel of the Fourier transform. The freedom in using various unitary equivalent representations is typical of quantum mechanics, but there is a natural distinguished representation in the case of integrable systems - Sklyanin's SoV representation. The SoV basis is an eigenbasis for a particularly interesting family of commuting operators [16-18]. BD integrals are related to non-compact conformal spin chains [8,22], and it is indeed possible to define a commuting family of operators $\mathbf{Q}(u)$, which includes the graph-building operator.

For a long time, application of the $\mathrm{SoV}$ method was restricted to the models with symmetry group of the lower rank [17, 22-25], or to the Toda chain [16, 26-29]. In the last few years, however, much progress has been made for compact [30-38] and non-compact [37, $39,40]$ spin chains with higher-rank symmetry.

In the present paper, we generalise the first step in the computation of $\mathrm{BD}$ integrals to the case of general $d$, and we construct the corresponding $d$-dimensional SoV representation. Namely, we give an explicit description of a basis of eigenvectors of $\mathbf{Q}(u)$ and analyse their properties, thus revealing an underlying realisation of the Zamolodchikovs-Faddeev algebra $[20,41]$ with respect to the exchange of their quantum numbers, i.e. the excitations of the lattice. The construction of the eigenvectors for an arbitrary number of sites extends, to any size $N$ of the fishnet, the two-site eigenvector presented in [42]. However, the investigation of the symmetry properties of the eigenvectors, the calculation of the corresponding inner product and of Sklyanin's measure are based on a new integral interchange relation. The main ingredient of this interchange relation is a particular $O(d)$-invariant R-matrix - more precisely, a solution of the Yang-Baxter equation acting in the tensor product of two arbitrary symmetric traceless representations of $O(d)$.

Applied to the computation of the $M \times N$ BD integral, our results allow us to rewrite it in the following way:

$$
\begin{aligned}
& \pi^{\frac{N d}{2}}\left\langle x, \ldots, x\left|\left(\prod_{i=1}^{N} \hat{x}_{i-1, i}^{2 \delta}\right) \mathbf{B}_{N, \tilde{\delta}}^{M+1}\right| y, \ldots, y\right\rangle \\
& =\sum_{\substack{0 \leqslant l_{1}, \ldots, l_{N} \leqslant+\infty \\
1 \leqslant m_{i} \leqslant d_{l_{i}}}} \int_{-\infty}^{+\infty} \cdots \int_{-\infty}^{+\infty}\left\langle x, \ldots, x\left|\left(\prod_{i=1}^{N} \hat{x}_{i-1, i}^{2 \delta}\right)\right| \mathbf{u}_{1}, \ldots, \mathbf{u}_{N} ; C_{m_{1}, l_{1}} \otimes \cdots \otimes C_{m_{N}, l_{N}}\right\rangle \\
& \\
& \left.\times\left\langle\mathbf{u}_{1}, \ldots, \mathbf{u}_{N} ; C_{m_{1}, l_{1}} \otimes \cdots \otimes C_{m_{N}, l_{N}} \mid y, \ldots, y\right\rangle \frac{\mu\left(\mathbf{u}_{1}, \ldots, \mathbf{u}_{N}\right)}{N !} \prod_{k=1}^{N} Q_{l_{k}}^{M+1}\left(u_{k}\right) \mathrm{d} u_{k}, \quad, 1.1\right)
\end{aligned}
$$


where $\mathbf{u}_{k}=\left(u_{k}, l_{k}\right)$, and $\left\{\left|\mathbf{u}_{1}, \ldots, \mathbf{u}_{N} ; C_{m_{1}, l_{1}} \otimes \cdots \otimes C_{m_{N}, l_{N}}\right\rangle\right\}$ is a complete basis of eigenvectors of the graph-building operator $\mathbf{B}_{N, \tilde{\delta}}$,

$$
Q_{l}(u)=\frac{\Gamma(\delta) \Gamma\left(\frac{d}{4}-\frac{\delta}{2}+\frac{l}{2}-\mathrm{i} u\right) \Gamma\left(\frac{d}{4}-\frac{\delta}{2}+\frac{l}{2}+\mathrm{i} u\right)}{\Gamma(\tilde{\delta}) \Gamma\left(\frac{d}{4}+\frac{\delta}{2}+\frac{l}{2}+\mathrm{i} u\right) \Gamma\left(\frac{d}{4}+\frac{\delta}{2}+\frac{l}{2}-\mathrm{i} u\right)},
$$

and Sklyanin's measure is

$$
\mu\left(\mathbf{u}_{1}, \ldots, \mathbf{u}_{N}\right)=\prod_{1 \leqslant j<k \leqslant N}\left[\left(u_{j}-u_{k}\right)^{2}+\frac{\left(l_{j}-l_{k}\right)^{2}}{4}\right]\left[\left(u_{j}-u_{k}\right)^{2}+\frac{\left(d-2+l_{j}+l_{k}\right)^{2}}{4}\right] .
$$

The explicit construction of the eigenvectors is described in section 3. The next technical step to be performed is the simplification of $\left\langle\mathbf{u}_{1}, \ldots, \mathbf{u}_{N} ; C_{m_{1}, l_{1}} \otimes \cdots \otimes C_{m_{N}, l_{N}} \mid y, \ldots, y\right\rangle$. Though this was quite easy in two and four dimensions $[12,14,15]$, we will show, on the simplest non-trivial example, i.e. the $N=2$ case, that the situation in generic dimension is much more involved.

The rest of the paper is organised as follows. In section 2, we work out the properties of a solution $\mathbb{R}_{l_{1}, l_{2}}(u)$ of the Yang-Baxter equation acting on the tensor product of spaces of rank- $l_{1}$ and rank- $l_{2}$ symmetric traceless tensors. In particular, we shall present two new representations for the matrix elements of the $O(d)$-invariant R-matrix. The first one is obtained by a direct application of the fusion procedure [43], while the second one is an integral representation and is in fact equivalent to the interchange relation we alluded to above. Along with that, the next section introduces the graphical Feynman diagram notations of lines and vertices that will be used to prove the most cumbersome identities throughout the paper. Section 3 contains the explicit construction of the eigenvectors of the fishnet, which is done in an iterative manner as in the $d=2$ case $[12,22,44]$, the determination of the spectrum, and the analysis of the eigenvectors' properties. An eigenvector for a lattice of size $N$ turns out to be described by a set of $N$ excitations, each characterised by a rapidity $u_{k}$ and a bound-state index or spin $l_{k}$, according to the analysis carried out in [42]. The rearrangement of the excitations inside an eigenvector and the overlap of eigenvectors reveal a picture of factorised scattering of excitations described by the $\mathrm{S}$ matrix $\mathbb{R}_{l_{1}, l_{2}}(u)$, up to a non-trivial phase. We work out, in section 4 , the simplest examples of application to Basso-Dixon integrals in any $d$.

Appendix A contains the basic integral identities used throughout the paper, while the proof of the integral representation for the R-matrix and of some related identities are relegated to appendices (B), (C), and (D). Appendix (E) presents an alternative basis of the eigenvectors which makes use of auxiliary spinors, in the spirit of the $d=4$ results of $[14,15]$.

\section{$2 O(d)$-invariant R Matrices}

For $l \in \mathbb{N}$, we denote by $\mathbb{V}_{l}$ the (complex) vector space of symmetric traceless tensors of rank $l$, in dimension $d$. We shall denote its dimension by $d_{l}$. We will sometimes refer to $l$ as the spin. 
This section contains an explicit construction of the R-matrices $\mathbb{R}_{l_{1}, l_{2}}$ acting in the tensor product $\mathbb{V}_{l_{1}} \otimes \mathbb{V}_{l_{2}}$ and satisfying the Yang-Baxter relation

$$
\mathbb{R}_{l_{1}, l_{2}}(u) \mathbb{R}_{l_{1}, l_{3}}(u+v) \mathbb{R}_{l_{2}, l_{3}}(v)=\mathbb{R}_{l_{2}, l_{3}}(v) \mathbb{R}_{l_{1}, l_{3}}(u+v) \mathbb{R}_{l_{1}, l_{2}}(u)
$$

in $\mathbb{V}_{l_{1}} \otimes \mathbb{V}_{l_{2}} \otimes \mathbb{V}_{l_{3}}$ for arbitrary $l_{1}, l_{2}, l_{3}$. Our starting point will be the R-matrix by A. Zamolodchikov and Al. Zamolodchikov [41]

$$
\forall C \in \mathbb{V}_{1} \otimes \mathbb{V}_{1}, \quad\left[\mathbb{R}_{1,1}(u) C\right]^{\mu \nu}=\frac{1}{u+\mathrm{i}}\left[u C^{\mu \nu}+\mathrm{i} C^{\nu \mu}-\frac{\mathrm{i} u}{u+\mathrm{i} \frac{d-2}{2}} C_{\rho}^{\rho} \delta^{\mu \nu}\right] .
$$

In the first part of the section, we apply the fusion procedure $[21,43]$ to the construction of the general R-matrix $\mathbb{R}_{l_{1}, l_{2}}$. The fusion procedure was used for the calculation of $\mathrm{R}$ matrices $\mathbb{R}_{1,2}, \mathbb{R}_{1,3}$ and $\mathbb{R}_{2,2}$ by N. MacKay [45] and for $\mathbb{R}_{1, l}$ by N. Reshetikhin [46, 47], we therefore generalise their results.

In the second part of the section, we shall prove an identity, which we call the interchange relation, and in which $\mathbb{R}_{l_{1}, l_{2}}$ plays the key role. This identity will be used extensively in the rest of the paper as it allows to prove symmetry properties of the eigenvectors of the transfer-matrix operators under the exchange of excitations. As a matter of fact, the interchange relation could be considered as the defining relation for the R-matrix $\mathbb{R}_{l_{1}, l_{2}}$, since it contains all the information about it. Starting from this identity, it is possible to derive an integral representation for the R-matrix, which allows to prove in a simple way its unitarity and the Yang-Baxter property. Vice versa, from the integral representation for the R-matrix it is possible to derive the interchange relation.

The equivalence of the two expressions for $\mathbb{R}_{l_{1}, l_{2}}$ - the integral representation and the representation obtained directly by fusion procedure - is far from obvious. Our proof is very technical and we postpone it to appendix (B). We should note that the spectral decomposition for the general R-matrix $\mathbb{R}_{l_{1}, l_{2}}$ was actually obtained thirty years ago by N. MacKay $[45,48]$. The latter result is in some sense complementary to both our expressions and we have checked their equivalence in the case of $\mathbb{R}_{1, l}$.

\subsection{Fusion procedure}

In this subsection we show that the R-matrix acting on $\mathbb{V}_{l_{1}} \otimes \mathbb{V}_{l_{2}}$ is defined by the following matrix elements

$$
\begin{aligned}
& x^{\otimes l_{1}} \otimes y^{\otimes l_{2}} \cdot\left[\mathbb{R}_{l_{1}, l_{2}}(u) \zeta^{\otimes l_{1}} \otimes \eta^{\otimes l_{2}}\right] \\
& =\frac{\left(\mathrm{i} u+\frac{l_{2}-l_{1}}{2}\right)_{l_{1}} \sum_{\substack{k \geqslant 0, n \geqslant 0 \\
k}} \frac{l_{1} ! l_{2} !}{k ! n !\left(l_{1}-k-n\right) !\left(l_{2}-k-n\right) !}}{\left(\mathrm{i} u \ln \left(l_{1}\right)_{l_{1}}, l_{2}\right)} \\
& \quad \times \frac{(x \cdot y \zeta \cdot \eta)^{k}(x \cdot \eta y \cdot \zeta)^{n}}{\left(\mathrm{i} u+\frac{4-l_{1}-l_{2}-d}{2}\right)_{k}\left(-\mathrm{i} u+\frac{2-l_{1}-l_{2}}{2}\right)_{n}}(x \cdot \zeta)^{l_{1}-k-n}(y \cdot \eta)^{l_{2}-k-n},
\end{aligned}
$$

where all contractions, represented with a dot, of tensor indices are done using the Euclidean metric $\delta_{\mu \nu}$, and $x, y, \zeta, \eta$ are four null vectors in $\mathbb{C}^{d}$. One has for instance

$$
\zeta^{2}=\zeta \cdot \zeta=\zeta_{\mu} \zeta^{\mu}=\zeta^{\mu} \zeta^{\nu} \delta_{\mu \nu}=0
$$


We also use the Pochhammer symbol

$$
(a)_{l}=\frac{\Gamma(a+l)}{\Gamma(a)}=\prod_{k=0}^{l-1}(a+k) .
$$

The proof of (2.3) is done in two steps. We first apply fusion to increase one of the spins, keeping the other equal to 1 . In that case, the previous formula contains only three terms and reads

$$
\begin{aligned}
x \otimes y^{\otimes l} \cdot\left[\mathbb{R}_{1, l}(u) \zeta \otimes \eta^{\otimes l}\right]= & \frac{1}{u+\mathrm{i} \frac{l+1}{2}}\left[\left(u-\mathrm{i} \frac{l-1}{2}\right) x \cdot \zeta(y \cdot \eta)^{l}\right. \\
& \left.+\mathrm{i} l x \cdot \eta y \cdot \zeta(y \cdot \eta)^{l-1}-\mathrm{i} l \frac{u-\mathrm{i} \frac{l-1}{2}}{u+\mathrm{i} \frac{d+l-3}{2}} x \cdot y \zeta \cdot \eta(y \cdot \eta)^{l-1}\right] .
\end{aligned}
$$

Equivalently, we could have written, for $C \in \mathbb{V}_{1} \otimes \mathbb{V}_{l}$,

$$
\begin{aligned}
{\left[\mathbb{R}_{1, l}(u) C\right]^{\mu \nu_{1} \cdots \nu_{l}}=} & \frac{1}{u+\mathrm{i} \frac{l+1}{2}}\left[\left(u-\mathrm{i} \frac{l-1}{2}\right) C^{\mu \nu_{1} \cdots \nu_{l}}+\mathrm{i} \sum_{j=1}^{l} C^{\nu_{j} \mu \nu \nu_{1} \cdots \widehat{\nu_{j} \cdots \nu_{l}}}\right. \\
& \left.-\mathrm{i} \frac{u-\mathrm{i} \frac{l-1}{2}}{u+\mathrm{i} \frac{d+l-3}{2}} \sum_{j=1}^{l} \delta^{\mu \nu_{j}} C^{\rho \rho \nu_{1} \cdots \widehat{\nu_{j} \cdots \nu_{l}}}+\frac{1}{u+\mathrm{i} \frac{d+l-3}{2}} \sum_{1 \leqslant j<k \leqslant l} \delta^{\nu_{j} \nu_{k}} C^{\rho \rho \mu \nu_{1} \cdots \widehat{\nu_{j}} \cdots \widehat{\nu_{k}} \cdots \nu_{l}}\right] .
\end{aligned}
$$

Before proving this last formula, we point out that, at the special point $u=\mathrm{i} \frac{l+1}{2}$, the matrix $\mathbb{R}_{1, l}(u)$ reduces to the orthogonal projector $\mathbb{P}_{1, l}^{(l+1)}$ onto $\mathbb{V}_{l+1} \subset \mathbb{V}_{1} \otimes \mathbb{V}_{l}$. This fact justifies why the fusion procedure gives new solutions of the Yang-Baxter relation. Its proof goes as follows: first, one notices from $(2.7)$ that $\mathbb{R}_{1, l}\left(\mathrm{i} \frac{l+1}{2}\right) C$ is symmetric traceless in all $l+1$ indices. After that, it is enough to remark that its contraction with any other symmetric traceless tensor $C^{\prime} \in \mathbb{V}_{l+1}$ is given by $C^{\prime} \cdot C$.

The proof is made by induction: the property (2.7) clearly holds for $l=1$ so we assume that it holds for some $l \geqslant 1$. Let us show it for $l+1$, where the fusion procedure states that

$$
\mathbb{P}_{1^{\prime}, l}^{(l+1)} \mathbb{R}_{1,1^{\prime}}\left(u-\frac{\mathrm{i} l}{2}\right) \mathbb{R}_{1, l}\left(u+\frac{\mathrm{i}}{2}\right) \mathbb{P}_{1^{\prime}, l}^{(l+1)}=\mathbb{R}_{1, l+1}(u) .
$$

We remind that, due to the Yang-Baxter equation, the left projector could be removed. Consequently, applying the left-hand side to $C \in \mathbb{V}_{1} \otimes \mathbb{V}_{l+1} \subset \mathbb{V}_{1} \otimes \mathbb{V}_{1}^{\prime} \otimes \mathbb{V}_{l}$ gives

$$
\begin{aligned}
& {\left[\mathbb{R}_{1,1^{\prime}}\left(u-\frac{\mathrm{i} l}{2}\right) \mathbb{R}_{1, l}\left(u+\frac{\mathrm{i}}{2}\right) C\right]^{\mu \nu_{1} \cdots \nu_{l+1}} \text { quer }} \\
& =\frac{1}{u+\mathrm{i} \frac{2-l}{2}}\left[\left(u-\frac{\mathrm{i} l}{2}\right)\left[\mathbb{R}_{1, l}\left(u+\frac{\mathrm{i}}{2}\right) C\right]^{\mu \nu_{1} \cdots \nu_{l+1}}\right. \\
& \left.\quad+\mathrm{i}\left[\mathbb{R}_{1, l}\left(u+\frac{\mathrm{i}}{2}\right) C\right]^{\nu_{1} \mu \nu_{2} \cdots \nu_{l+1}}-\mathrm{i} \frac{u-\frac{\mathrm{i} l}{2}}{u+\mathrm{i} \frac{d-2-l}{2}}\left[\mathbb{R}_{1, l}\left(u+\frac{\mathrm{i}}{2}\right) C\right]^{\rho \rho \nu_{2} \cdots \nu_{l+1}} \delta^{\mu \nu_{1}}\right] .
\end{aligned}
$$


We now use equation (2.7) to write the second term in the right-hand side as

$$
\begin{aligned}
& {\left[\mathbb{R}_{1, l}\left(u+\frac{\mathrm{i}}{2}\right) C\right]^{\nu_{1} \mu \nu_{2} \cdots \nu_{l+1}}} \\
& =\frac{1}{u+\mathrm{i} \frac{l+2}{2}}\left[\left(u-\mathrm{i} \frac{l-2}{2}\right) C^{\nu_{1} \mu \nu_{2} \cdots \nu_{l+1}}+\mathrm{i} \sum_{j=2}^{l+1} C^{\nu_{j} \mu \nu_{1} \cdots \widehat{\nu_{j} \cdots \nu_{l+1}}}\right. \\
& \left.\quad-\mathrm{i} \frac{u-\mathrm{i} \frac{l-2}{2}}{u+\mathrm{i} \frac{d+l-2}{2}} \sum_{j=2}^{l+1} \delta^{\nu_{1} \nu_{j}} C^{\rho \mu \rho \nu_{2} \cdots \widehat{\nu_{j}} \cdots \nu_{l+1}}+\frac{1}{u+\mathrm{i} \frac{d+l-2}{2}} \sum_{2 \leqslant j<k \leqslant l+1} \delta^{\nu_{j} \nu_{k}} C^{\rho \mu \rho \nu_{1} \cdots \widehat{\nu_{j}} \cdots \widehat{\nu_{k}} \cdots \nu_{l+1}}\right],
\end{aligned}
$$

and, using the fact that $C$ is symmetric traceless in the last $l+1$ indices, the third term is

$$
\left[\mathbb{R}_{1, l}\left(u+\frac{\mathrm{i}}{2}\right) C\right]^{\rho \rho \nu_{2} \cdots \nu_{l+1}}=\frac{\left(u-\mathrm{i} \frac{l-2}{2}\right)\left(u+\mathrm{i} \frac{d-l-2}{2}\right)}{\left(u+\mathrm{i} \frac{l+2}{2}\right)\left(u+\mathrm{i} \frac{d+l-2}{2}\right)} C^{\rho \rho \nu_{2} \cdots \nu_{l+1}} .
$$

Putting everything together we straightforwardly recover $(2.7)$ for $\mathbb{R}_{1, l+1}(u)$. Turning our attention to the more general case, it suffices to prove $(2.3)$ for $l_{1} \leqslant l_{2}$, which we shall do by induction on $l_{1}$ for given $l_{2}$. We have just verified it for $l_{1}=1$, and assuming it holds for some $l_{1} \leqslant l_{2}-1$, one just needs to use fusion to compute $\mathbb{R}_{l_{1}+1, l_{2}}(u)$ :

$$
\begin{aligned}
& x^{\otimes l_{1}+1} \otimes y^{\otimes l_{2}} \cdot \mathbb{R}_{l_{1}, l_{2}}\left(u+\frac{\mathrm{i}}{2}\right) \mathbb{R}_{1, l_{2}}\left(u-\frac{\mathrm{i} l_{1}}{2}\right) \zeta^{\otimes l_{1}+1} \otimes \eta^{\otimes l_{2}} \\
& =x^{\otimes l_{1}+1} \otimes y^{\otimes l_{2}} \cdot \mathbb{R}_{l_{1}+1, l_{2}}(u) \zeta^{\otimes l_{1}+1} \otimes \eta^{\otimes l_{2}} .
\end{aligned}
$$

In the previous equation the product of the two R-matrices is taken in $\mathbb{V}_{l_{2}}$. In order to compute this product, one may insert a resolution of the identity of $\mathbb{V}_{l_{2}}$ between the two matrices. More explicitly, if $\left\{C_{j, l}\right\}_{1 \leqslant j \leqslant d_{l}}$ is an orthonormal basis of $\mathbb{V}_{l}$ (for the inner product $\left.\left(C, C^{\prime}\right)=C_{\mu_{1} \ldots \mu_{l}}^{*} C^{\prime \mu_{1} \ldots \mu_{l}}=C^{*} \cdot C^{\prime}\right)$, one can write

$$
\begin{aligned}
& x^{\otimes l_{1}+1} \otimes y^{\otimes l_{2}} \cdot \mathbb{R}_{l_{1}, l_{2}}(a) \mathbb{R}_{1, l_{2}}(b) \zeta^{\otimes l_{1}+1} \otimes \eta^{\otimes l_{2}} \\
& =\sum_{j=1}^{d_{l_{2}}} x^{\otimes l_{1}} \otimes y^{\otimes l_{2}} \cdot\left[\mathbb{R}_{l_{1}, l_{2}}(a) \zeta^{\otimes l_{1}} \otimes C_{j, l_{2}}\right] x \otimes C_{j, l_{2}}^{*} \cdot\left[\mathbb{R}_{1, l_{2}}(b) \zeta \otimes \eta^{\otimes l_{2}}\right] .
\end{aligned}
$$

Thus, according to the formulas (2.6) and (2.3), we can write

$$
\begin{gathered}
x^{\otimes l_{1}+1} \otimes y^{\otimes l_{2}} \cdot \mathbb{R}_{l_{1}, l_{2}}\left(u+\frac{\mathrm{i}}{2}\right) \mathbb{R}_{1, l_{2}}\left(u-\frac{\mathrm{i} l_{1}}{2}\right) \zeta^{\otimes l_{1}+1} \otimes \eta^{\otimes l_{2}} \\
=\frac{\left(\mathrm{i} u+\frac{l_{2}-l_{1}-1}{2}\right)_{l_{1}+1}}{\left(\mathrm{i} u-\frac{l_{1}+l_{2}+1}{2}\right)_{l_{1}+1}} \sum_{k+n \leqslant l_{1}} \frac{l_{1} ! l_{2} !}{k ! n !\left(l_{1}-k-n\right) !\left(l_{2}-k-n\right) !} \frac{(x \cdot y)^{k}(y \cdot \zeta)^{n}(x \cdot \zeta)^{l_{1}-k-n}}{\left(\mathrm{i} u+\frac{3-l_{1}-l_{2}-d}{2}\right)_{k}\left(-\mathrm{i} u+\frac{3-l_{1}-l_{2}}{2}\right)_{n}} \\
\sum_{j=1}^{d_{l_{2}}\left(\zeta^{\otimes k} \otimes x^{\otimes n} \otimes y^{\otimes\left(l_{2}-k-n\right)} \cdot C_{j, l_{2}}\right)}\left[\begin{array}{c}
x \cdot \zeta\left(C_{j, l_{2}}^{*} \cdot \eta^{\otimes l_{2}}\right)+\frac{l x \cdot \eta\left(C_{j, l_{2}}^{*} \cdot \zeta \otimes \eta^{\otimes\left(l_{2}-1\right)}\right)}{-\mathrm{i} u+\frac{1-l_{1}-l_{2}}{2}} \\
\left.+\frac{l \zeta \cdot \eta\left(C_{j, l_{2}}^{*} \cdot x \otimes \eta^{\otimes\left(l_{2}-1\right)}\right)}{\mathrm{i} u+\frac{3+l_{1}-l_{2}-d}{2}}\right] .
\end{array}\right.
\end{gathered}
$$


The only additional formulas needed in order to conclude the proof are

$$
\sum_{j=1}^{d_{l_{2}}}\left(\zeta^{\otimes k} \otimes x^{\otimes n} \otimes y^{\otimes\left(l_{2}-k-n\right)} \cdot C_{j, l_{2}}\right)\left(C_{j, l_{2}}^{*} \cdot \eta^{\otimes l_{2}}\right)=(\eta \cdot \zeta)^{k}(\eta \cdot x)^{n}(\eta \cdot y)^{l_{2}-k-n},
$$

and

$$
\begin{aligned}
& \sum_{j=1}^{d_{l_{2}}}\left(\zeta^{\otimes k} \otimes x^{\otimes n} \otimes y^{\otimes\left(l_{2}-k-n\right)} \cdot C_{j, l_{2}}\right)\left(C_{j, l_{2}}^{*} \cdot \theta \otimes \eta^{\otimes\left(l_{2}-1\right)}\right) \\
& =\frac{(\eta \cdot \zeta)^{k-1}(\eta \cdot x)^{n-1}(\eta \cdot y)^{l_{2}-k-n-1}}{l_{2}} \\
& \quad \times\left[\left(l_{2}-k-n\right)(y \cdot \theta)(x \cdot \eta)(\zeta \cdot \eta)+n(x \cdot \theta)(y \cdot \eta)(\zeta \cdot \eta)+k(\zeta \cdot \theta)(y \cdot \eta)(x \cdot \eta)\right. \\
& \quad-\frac{2 \theta \cdot \eta}{d+2\left(l_{2}-2\right)}\left[\left(l_{2}-k-n\right) n(y \cdot x)(\zeta \cdot \eta)+\left(l_{2}-k-n\right) k(y \cdot \zeta)(x \cdot \eta)+k n(x \cdot \zeta)(y \cdot \eta)\right],
\end{aligned}
$$

where $\theta^{2}=0$, and we will apply it to $\theta \in\{\zeta, x\}$. The first one is trivial since $\eta_{2}^{\otimes l_{2}} \in \mathbb{V}_{l_{2}}$, and $\left\{C_{j, l_{2}}\right\}_{1 \leqslant j \leqslant d_{l_{2}}}$ is an orthonormal basis of $\mathbb{V}_{l_{2}}$. The second one is a consequence of

$$
\begin{aligned}
\sum_{j=1}^{d_{l_{2}}} C_{j, l_{2}}^{\mu_{1} \cdots \mu_{l_{2}}}\left(C_{j, l_{2}}^{*} \cdot \theta \otimes \eta^{\otimes\left(l_{2}-1\right)}\right)= & \frac{1}{l_{2}}\left[\sum_{i=1}^{l_{2}} \theta^{\mu_{i}} \eta^{\mu_{1}} \ldots \widehat{\eta^{\mu_{i}}} \ldots \eta^{\mu_{l_{2}}}\right. \\
& \left.-\frac{2}{d+2\left(l_{2}-2\right)} \sum_{1 \leqslant i<j \leqslant l_{2}} \theta \cdot \eta \delta^{\mu_{i} \mu_{j}} \eta^{\mu_{1}} \ldots \widehat{\eta^{\mu_{i}}} \ldots \widehat{\eta^{\mu_{j}}} \ldots \eta^{\mu_{l_{2}}}\right]
\end{aligned}
$$

which is the orthogonal projection of $\theta \otimes \eta^{\otimes\left(l_{2}-1\right)} \in \mathbb{V}_{1} \otimes \mathbb{V}_{l_{2}-1}$ onto $\mathbb{V}_{l_{2}} \subset \mathbb{V}_{1} \otimes \mathbb{V}_{l_{2}-1}$, as explained at the beginning of this section (recall that this projector is nothing else than $\left.\mathbb{R}_{1, l_{2}-1}\left(\mathrm{i} \frac{l_{2}}{2}\right)\right)$.

Using the two additional formulas, we can compute the sum over $d_{l_{2}}$ appearing in (2.14):

$$
\begin{aligned}
& \sum_{j=1}^{d_{l_{2}}}\left(\zeta^{\otimes k} \otimes x^{\otimes n} \otimes y^{\otimes\left(l_{2}-k-n\right)} \cdot C_{j, l_{2}}\right)\left[x \cdot \zeta\left(C_{j, l_{2}}^{*} \cdot \eta^{\otimes l_{2}}\right)\right. \\
& \left.+\frac{l x \cdot \eta\left(C_{j, l_{2}}^{*} \cdot \zeta \otimes \eta^{\otimes\left(l_{2}-1\right)}\right)}{-\mathrm{i} u+\frac{1-l_{1}-l_{2}}{2}}+\frac{l \zeta \cdot \eta\left(C_{j, l_{2}}^{*} \cdot x \otimes \eta^{\otimes\left(l_{2}-1\right)}\right)}{\mathrm{i} u+\frac{3+l_{1}-l_{2}-d}{2}}\right] \\
& =\frac{(\eta \cdot \zeta)^{k}(\eta \cdot x)^{n}(\eta \cdot y)^{l_{2}-k-n-1}}{\left(-\mathrm{i} u+\frac{1-l_{1}-l_{2}}{2}\right)\left(\mathrm{i} u+\frac{3+l_{1}-l_{2}-d}{2}\right)}\left[(x \cdot \zeta)(\eta \cdot y)\left(-\mathrm{i} u+\frac{1-l_{1}-l_{2}}{2}+n\right)\right. \\
& \quad \times\left(\mathrm{i} u+\frac{3+l_{1}-l_{2}-d}{2}+k\right)+(y \cdot \zeta)(\eta \cdot x)\left(l_{2}-k-n\right)\left(\mathrm{i} u+\frac{3+l_{1}-l_{2}-d}{2}+k\right) \\
& \left.\quad+(y \cdot x)(\eta \cdot \zeta)\left(l_{2}-k-n\right)\left(-\mathrm{i} u+\frac{1-l_{1}-l_{2}}{2}+n\right)\right]
\end{aligned}
$$


After plugging (2.19) back into (2.14), we proceed to rewriting the sum $\sum_{k+n \leqslant l_{1}}$ into a sum $\sum_{k^{\prime}+n^{\prime} \leqslant l_{1}+1}$. The terms contributing to a given pair $\left(k^{\prime}, n^{\prime}\right)$ come from $(k, n) \in$ $\left\{\left(k^{\prime}, n^{\prime}\right),\left(k^{\prime}-1, n^{\prime}\right),\left(k^{\prime}, n^{\prime}-1\right)\right\}$. When $(k, n)=\left(k^{\prime}, n^{\prime}\right)$, the contribution (without the tensors) is

$$
\frac{l_{1} ! l_{2} !}{k^{\prime} ! n^{\prime} !\left(l_{1}+1-k^{\prime}-n^{\prime}\right) !\left(l_{2}-k^{\prime}-n^{\prime}\right) !} \frac{l_{1}+1-k^{\prime}-n^{\prime}}{\mathrm{i} u+\frac{3+l_{1}-l_{2}-d}{2}} \frac{\mathrm{i} u+\frac{3+l_{1}-l_{2}-d}{2}+k^{\prime}}{\left(\mathrm{i} u+\frac{3-l_{1}-l_{2}-d}{2}\right)_{k^{\prime}}\left(-\mathrm{i} u+\frac{1-l_{1}-l_{2}}{2}\right)_{n^{\prime}}}
$$

while when $(k, n)=\left(k^{\prime}-1, n^{\prime}\right)$ it is

$$
\frac{l_{1} ! l_{2} !}{k^{\prime} ! n^{\prime} !\left(l_{1}+1-k^{\prime}-n^{\prime}\right) !\left(l_{2}-k^{\prime}-n^{\prime}\right) !} \frac{k^{\prime}}{\mathrm{i} u+\frac{3+l_{1}-l_{2}-d}{2}} \frac{1}{\left(\mathrm{i} u+\frac{3-l_{1}-l_{2}-d}{2}\right)_{k^{\prime}-1}\left(-\mathrm{i} u+\frac{1-l_{1}-l_{2}}{2}\right)_{n^{\prime}}},
$$

and, when $(k, n)=\left(k^{\prime}, n^{\prime}-1\right)$, it is

$$
\frac{l_{1} ! l_{2} !}{k^{\prime} ! n^{\prime} !\left(l_{1}+1-k^{\prime}-n^{\prime}\right) !\left(l_{2}-k^{\prime}-n^{\prime}\right) ! \mathrm{i} u+\frac{3+l_{1}-l_{2}-d}{2}} \frac{\mathrm{i} u+\frac{3+l_{1}-l_{2}-d}{2}+k^{\prime}}{\left(\mathrm{i} u+\frac{3-l_{1}-l_{2}-d}{2}\right)_{k^{\prime}}\left(-\mathrm{i} u+\frac{1-l_{1}-l_{2}}{2}\right)_{n^{\prime}}} \text {. }
$$

The sum of the previous three terms is

$$
\frac{\left(l_{1}+1\right) ! l_{2} !}{k^{\prime} ! n^{\prime} !\left(l_{1}+1-k^{\prime}-n^{\prime}\right) !\left(l_{2}-k^{\prime}-n^{\prime}\right) !} \frac{1}{\left(\mathrm{i} u+\frac{3-l_{1}-l_{2}-d}{2}\right)_{k^{\prime}}\left(-\mathrm{i} u+\frac{1-l_{1}-l_{2}}{2}\right)_{n^{\prime}}},
$$

which concludes the proof of $(2.3)$ for $\left(l_{1}+1, l_{2}\right)$.

Extension of (2.3) to symmetric tensors. We now want to compute $x^{\otimes l_{1}} \otimes y^{\otimes l_{2}}$. $\left[\mathbb{R}_{l_{1}, l_{2}}(u) \zeta^{\otimes l_{1}} \otimes \eta^{\otimes l_{2}}\right]$ when $\zeta^{2}=\eta^{2}=0$, but $x^{2} \neq 0$ and $y^{2} \neq 0$. Since $\mathbb{R}_{l_{1}, l_{2}}(u) \zeta^{\otimes l_{1}} \otimes \eta^{\otimes l_{2}}$ belongs to $\mathbb{V}_{l_{1}} \otimes \mathbb{V}_{l_{2}}$, only the symmetric traceless parts of $x^{\otimes l_{1}}$ and $y^{\otimes l_{2}}$ are needed. Let us call $X_{l}$ the symmetric traceless part of $x^{\otimes l}$, it is given by

$$
X_{l}^{\mu_{1} \cdots \mu_{l}}=\sum_{p=0}^{\left\lfloor\frac{l}{2}\right\rfloor} \frac{\left(x^{2}\right)^{p}}{\left(2-l-\frac{d}{2}\right)_{p} 2^{p}} \sum_{\left\{i_{1}, j_{1}\right\}, \ldots,\left\{i_{p}, j_{p}\right\}} \prod_{k=1}^{p} \delta^{\mu_{i_{k}} \mu_{j_{k}}} \prod_{i \notin\left\{i_{1}, j_{1}, \ldots, i_{p}, j_{p}\right\}} x^{\mu_{i}},
$$

where, for a given $p$, we sum over $\frac{l !}{(l-2 p) ! p ! 2^{p}}$ possible ways of forming $p$ pairs among $l$ elements. We can thus write

$$
x^{\otimes l_{1}} \otimes y^{\otimes l_{2}} \cdot\left[\mathbb{R}_{l_{1}, l_{2}}(u) \zeta^{\otimes l_{1}} \otimes \eta^{\otimes l_{2}}\right]=X_{l_{1}} \otimes Y_{l_{2}} \cdot\left[\mathbb{R}_{l_{1}, l_{2}}(u) \zeta^{\otimes l_{1}} \otimes \eta^{\otimes l_{2}}\right],
$$

and then use the formula (2.3). 
To start with, we consider only one vector that is not null: $\alpha^{2}=0$ but $y^{2} \neq 0$, so that we have

$$
\begin{aligned}
& \alpha^{\otimes l_{1}} \otimes y^{\otimes l_{2}} \cdot\left[\mathbb{R}_{l_{1}, l_{2}}(u) \zeta^{\otimes l_{1}} \otimes \eta^{\otimes l_{2}}\right] \\
& =\frac{\left(\mathrm{i} u+\frac{l_{2}-l_{1}}{2}\right)_{l_{1}}}{\left(\mathrm{i} u-\frac{l_{1}+l_{2}}{2}\right)_{l_{1}}} \sum_{k, n \geqslant 0} \frac{\left(-l_{1}\right)_{k+n}\left(-l_{2}\right)_{k+n}}{k ! n !} \\
& \quad \times \frac{(\zeta \cdot \eta)^{k}(\alpha \cdot \eta)^{n}(\alpha \cdot \zeta)^{l_{2}-k-n}}{\left(\mathrm{i} u+\frac{4-l_{1}-l_{2}-d}{2}\right)_{k}\left(-\mathrm{i} u+\frac{2-l_{1}-l_{2}}{2}\right)_{n}}\left(Y_{l_{2}} \cdot \alpha^{\otimes k} \otimes \zeta^{\otimes n} \otimes \eta^{\otimes\left(l_{2}-k-n\right)}\right) .
\end{aligned}
$$

We then use the explicit expression for $Y_{l_{2}}$ to compute

$$
\begin{aligned}
Y_{l_{2}} \cdot \alpha^{\otimes k} \otimes \zeta^{\otimes n} \otimes \eta^{\otimes\left(l_{2}-k-n\right)} & \\
= & \sum_{q=0}^{\left\lfloor\frac{l_{2}}{2}\right\rfloor} \sum_{a+b \leqslant q} \frac{(-k)_{a+b}(-n)_{q-b}\left(n+k-l_{2}\right)_{q-a}}{a ! b !(q-a-b) !} \frac{\left(y^{2}\right)^{q}}{\left(2-l_{2}-\frac{d}{2}\right)_{q} 2^{q}} \\
& (\alpha \cdot \zeta)^{a}(\alpha \cdot \eta)^{b}(\zeta \cdot \eta)^{q-a-b}(\alpha \cdot y)^{k-a-b}(y \cdot \zeta)^{n+b-q}(y \cdot \eta)^{l_{2}+a-k-n-q},
\end{aligned}
$$

which implies

$$
\begin{aligned}
\alpha^{\otimes l_{1}} \otimes y^{\otimes l_{2}} \cdot\left[\mathbb{R}_{l_{1}, l_{2}}(u) \zeta^{\otimes l_{1}} \otimes \eta^{\otimes l_{2}}\right] \\
\left.=\frac{\left(\mathrm{i} u+\frac{l_{2}-l_{1}}{2}\right)_{l_{1}} \sum_{\left.\frac{l_{2}}{2}\right\rfloor} \sum_{\substack{K, N \geqslant q \\
2}} \frac{(-1)^{q+a}\left(-l_{1}\right)_{K+N+a-q}\left(-l_{2}\right)_{K+N}}{(K-q) !(N-q) ! a ! b !(q-a-b) !}}{}\right)^{\frac{(\alpha \cdot \zeta)^{l_{1}+q-N-K}(\alpha \cdot \eta)^{N}(\zeta \cdot \eta)^{K}\left(y^{2}\right)^{q}(\alpha \cdot y)^{K-q}(y \cdot \zeta)^{N-q}(y \cdot \eta)^{l_{2}-N-K}}{2+b \leqslant q}} \\
2^{q}\left(2-l_{2}-\frac{d}{2}\right)_{q}\left(\mathrm{i} u+\frac{4-l_{1}-l_{2}-d}{2}\right)_{K+a+b-q}\left(-\mathrm{i} u+\frac{2-l_{1}-l_{2}}{2}\right)_{N-b}
\end{aligned}
$$

where we have changed summation indices from $k, n$ to $K=k+q-a-b$ and $N=n+b$. Recalling the Gauss identity

$$
\sum_{k=0}^{n} \frac{(-n)_{k}(u)_{k}}{k !(v)_{k}}=\frac{(v-u)_{n}}{(v)_{n}}
$$

one can perform the sums over $a$ and $b$

$$
\begin{aligned}
& \sum_{a+b \leqslant q} \frac{(-1)^{q+a}\left(-l_{1}\right)_{K+N+a-q}}{a ! b !(q-a-b) !} \frac{1}{\left(\mathrm{i} u+\frac{4-l_{1}-l_{2}-d}{2}\right)_{K+a+b-q}\left(-\mathrm{i} u+\frac{2-l_{1}-l_{2}}{2}\right)_{N-b}} \\
& =\frac{1}{\left(\mathrm{i} u+\frac{4-l_{1}-l_{2}-d}{2}\right)_{K}\left(-\mathrm{i} u+\frac{2-l_{1}-l_{2}}{2}\right)_{N}} \sum_{a=0}^{q} \frac{\left(-l_{1}\right)_{K+N+a-q}\left(\frac{d}{2}+l_{1}+l_{2}-K-N-1\right)_{q-a}}{a !(q-a) !} \\
& =\frac{\left(-l_{1}\right)_{K+N-q}}{q !} \frac{(-1)^{q}\left(2-l_{2}-\frac{d}{2}\right)_{q}}{\left(\mathrm{i} u+\frac{4-l_{1}-l_{2}-d}{2}\right)_{K}\left(-\mathrm{i} u+\frac{2-l_{1}-l_{2}}{2}\right)_{N}}
\end{aligned}
$$




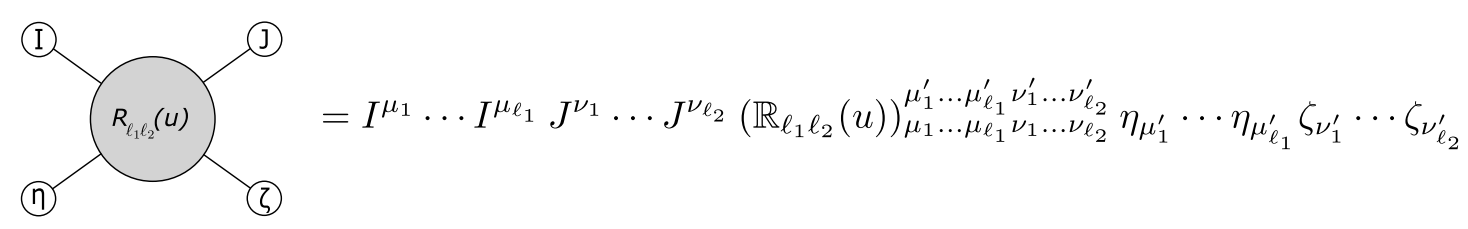

Figure 2. Graphical representation of a matrix element of the $O(d)$-invariant $\mathbb{R}$-matrix $\mathbb{R}_{l_{1}, l_{2}}(u)$.

and eventually get

$$
\begin{aligned}
& \alpha^{\otimes l_{1}} \otimes y^{\otimes l_{2}} \cdot\left[\mathbb{R}_{l_{1}, l_{2}}(u) \zeta^{\otimes l_{1}} \otimes \eta^{\otimes l_{2}}\right] \\
& =\frac{\left(\mathrm{i} u+\frac{l_{2}-l_{1}}{2}\right)_{l_{1}} \sum^{\left\lfloor\frac{l_{2}}{2}\right\rfloor} \sum_{q=0} \frac{\left(-l_{1}\right)_{K+N-q}\left(-l_{2}\right)_{K+N}(-N)_{q}}{\left(\mathrm{i} u-\frac{l_{1}+l_{2}}{2}\right)_{l_{1}}}}{q !(K-q) ! N !} \\
& \frac{\left(y^{2}\right)^{q}(\alpha \cdot \zeta)^{l_{1}+q-N-K}(\alpha \cdot \eta)^{N}(\zeta \cdot \eta)^{K}(\alpha \cdot y)^{K-q}(y \cdot \zeta)^{N-q}(y \cdot \eta)^{l_{2}-N-K}}{2^{q}\left(\mathrm{i} u+\frac{4-l_{1}-l_{2}-d}{2}\right)_{K}\left(-\mathrm{i} u+\frac{2-l_{1}-l_{2}}{2}\right)_{N}} .
\end{aligned}
$$

The same procedure allows to compute $x^{\otimes l_{1}} \otimes y^{\otimes l_{2}} \cdot\left[\mathbb{R}_{l_{1}, l_{2}}(u) \zeta^{\otimes l_{1}} \otimes \eta^{\otimes l_{2}}\right]$. In this case we start from the expression

$$
\begin{aligned}
& X_{l_{1}} \cdot y^{\otimes(K-q)} \otimes \zeta^{\otimes\left(l_{1}+q-N-K\right)} \otimes \eta^{\otimes N} \\
& =\sum_{p=0}^{\left\lfloor\frac{l_{1}}{2}\right\rfloor} \sum_{a+b+c \leqslant p} \frac{(-K+q)_{2 p-a-b-2 c}\left(N+K-l_{1}-q\right)_{b+c}(-N)_{a+c}}{2^{p-a-b-c} a ! b ! c !(p-a-b-c) !} \frac{\left(x^{2}\right)^{p}\left(y^{2}\right)^{p-a-b-c}}{\left(2-l_{1}-\frac{d}{2}\right)_{p} 2^{p}} \\
& \quad \times(y \cdot \eta)^{a}(y \cdot \zeta)^{b}(\zeta \cdot \eta)^{c}(x \cdot y)^{K-q+2 c+a+b-2 p}(x \cdot \zeta)^{l_{1}+q-N-K-b-c}(x \cdot \eta)^{N-a-c}
\end{aligned}
$$

and, after the change of summation indices $q^{\prime}=q+p-a-b-c, k=K+c, n=N+p-a-c$, the sums over $a, b$, and $c$ can be performed via the repeated application of (2.29). One eventually obtains

$$
\begin{aligned}
& x^{\otimes l_{1}} \otimes y^{\otimes l_{2}} \cdot\left[\mathbb{R}_{l_{1}, l_{2}}(u) \zeta^{\otimes l_{1}} \otimes \eta^{\otimes l_{2}}\right]
\end{aligned}
$$

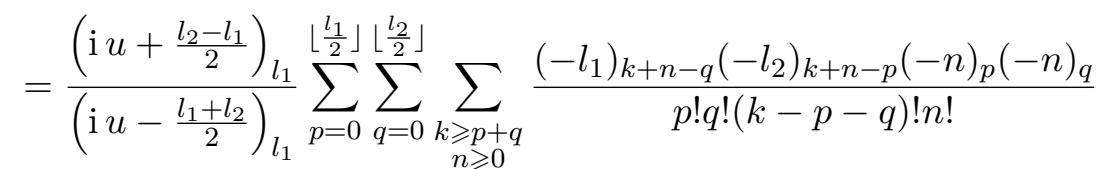

$$
\begin{aligned}
& \times\left(\frac{x^{2}}{2}\right)^{p}\left(\frac{y^{2}}{2}\right)^{q} \frac{(x \cdot \zeta)^{l_{1}+q-n-k}(x \cdot \eta)^{n-p}(\zeta \cdot \eta)^{k}(x \cdot y)^{k-p-q}(y \cdot \zeta)^{n-q}(y \cdot \eta)^{l_{2}+p-n-k}}{\left(\mathrm{i} u+\frac{4-l_{1}-l_{2}-d}{2}\right)_{k}\left(-\mathrm{i} u+\frac{2-l_{1}-l_{2}}{2}\right)_{n}} .
\end{aligned}
$$

In what follows, we shall use the graphical representation of the R-matrix shown in figure 2 .

\subsection{Spectral decomposition}

The spectral decomposition of the R-matrix was computed by N. MacKay [45, 48]. Since it is clear from our expression (2.3) that the completely symmetric traceless tensors are 
eigenvectors with eigenvalue 1, the normalisation is fixed and MacKay's result reads

$$
\mathbb{R}_{l_{1}, l_{2}}(u)=\sum_{0 \leqslant m \leqslant n \leqslant \min \left(l_{1}, l_{2}\right)} \prod_{p=1}^{m} \frac{u-\mathrm{i} \frac{d+l_{1}+l_{2}-2-2 q}{2}}{u+\mathrm{i} \frac{d+l_{1}+l_{2}-2-2 q}{2}} \prod_{q=1}^{n} \frac{u-\mathrm{i} \frac{l_{1}+l_{2}+2-2 q}{2}}{u+\mathrm{i} \frac{l_{1}+l_{2}+2-2 q}{2}} \mathbb{P}_{l_{1}, l_{2}}^{\left(l_{1}+l_{2}-2 n, n-m\right)},
$$

where $\mathbb{P}_{l_{1}, l_{2}}^{\left(n_{1}, n_{2}\right)}$ is the projector onto the subrepresentation with highest weight $n_{1} \omega_{1}+n_{2} \omega_{2}$ of $\mathbb{V}_{l_{1}} \otimes \mathbb{V}_{l_{2}}$, the $\omega_{a}$ 's being fundamental weights $\left(\mathbb{V}_{l}\right.$ has highest weight $\left.l \omega_{1}\right)$. When one of the spins is equal to one, the previous decomposition reads

$$
\mathbb{R}_{1, l}(u)=\mathbb{P}_{1, l}^{(l+1,0)}+\frac{u-\mathrm{i} \frac{l+1}{2}}{u+\mathrm{i} \frac{l+1}{2}} \mathbb{P}_{1, l}^{(l-1,1)}+\frac{u-\mathrm{i} \frac{d+l-3}{2}}{u+\mathrm{i} \frac{d+l-3}{2}} \frac{u-\mathrm{i} \frac{l+1}{2}}{u+\mathrm{i} \frac{l+1}{2}} \mathbb{P}_{1, l}^{(l-1,0)} .
$$

Let us check that this coincides with the expression (2.7) for the R-matrix. We first introduce some operators $\mathcal{P}, \mathcal{K}_{1}$, and $\mathcal{K}_{2}$, in terms of which the R-matrix reads

$$
\mathbb{R}_{1, l}(u)=\frac{1}{u+\mathrm{i} \frac{l+1}{2}}\left[\left(u-\mathrm{i} \frac{l-1}{2}\right) \mathrm{Id}+\mathrm{i} \mathcal{P}-\mathrm{i} \frac{u-\mathrm{i} \frac{l-1}{2}}{u+\mathrm{i} \frac{d+l-3}{2}} \mathcal{K}_{1}+\frac{1}{u+\mathrm{i} \frac{d+l-3}{2}} \mathcal{K}_{2}\right] .
$$

We have already explained that $\mathbb{P}_{1, l}^{(l+1,0)}=\mathbb{R}_{1, l}\left(\mathrm{i} \frac{l+1}{2}\right)$, and in terms of the new operators this reads

$$
\mathbb{P}_{1, l}^{(l+1,0)}=\frac{1}{l+1}\left[\mathrm{Id}+\mathcal{P}-\frac{2}{d+2 l-2}\left(\mathcal{K}_{1}+\mathcal{K}_{2}\right)\right] .
$$

We claim that the other two projectors are given by

$$
\mathbb{P}_{1, l}^{(l-1,1)}=\frac{1}{l+1}\left[l \mathrm{Id}-\mathcal{P}+\frac{1}{d+l-3}\left(2 \mathcal{K}_{2}-(l-1) \mathcal{K}_{1}\right)\right]
$$

and

$$
\mathbb{P}_{1, l}^{(l-1,0)}=\frac{1}{(d+2 l-2)(d+l-3)}\left[(d+2 l-4) \mathcal{K}_{1}-2 \mathcal{K}_{2}\right] .
$$

It is clear that $\mathbb{P}_{1, l}^{(l+1,0)}+\mathbb{P}_{1, l}^{(l-1,1)}+\mathbb{P}_{1, l}^{(l-1,0)}=\mathrm{Id}$, and that (2.35) is equivalent to (2.36). It remains to check that they are indeed orthogonal projectors, which leads to a tedious but straightforward computation that we do not show here.

\subsection{Interchange relation and integral representation}

In this section, we shall consider the main interchange relation drawn in figure 4 according to the graphical notation of figure 3. This interchange relation is crucial to the proof of the symmetry of the eigenvectors with respect to the exchange of the excitations numbers - explained in the following section.

Here and in the rest of the paper we will use the notation $\tilde{a} \equiv d / 2-a$, and we define a few standard functions of $u$ :

$$
\alpha(u)=\frac{\tilde{\delta}}{2}-\mathrm{i} u, \quad \beta(u)=\frac{\tilde{\delta}}{2}+\mathrm{i} u, \quad \text { so that } \quad \alpha(u)+\beta(u)+\delta=\frac{d}{2}, \quad \delta \in i \mathbb{R} .
$$

We define the powers of solid lines of the two squares in the left-hand side of figure 4 to be

$$
a_{j}=\tilde{\beta}_{j}-\frac{l_{j}}{2}, b_{j}=\alpha_{j}+\frac{d+l_{j}}{2}-1, c_{j}=\tilde{\alpha}_{j}+\frac{l_{j}}{2}, d_{j}=1-\tilde{\beta}_{j}-\frac{l_{j}}{2},
$$




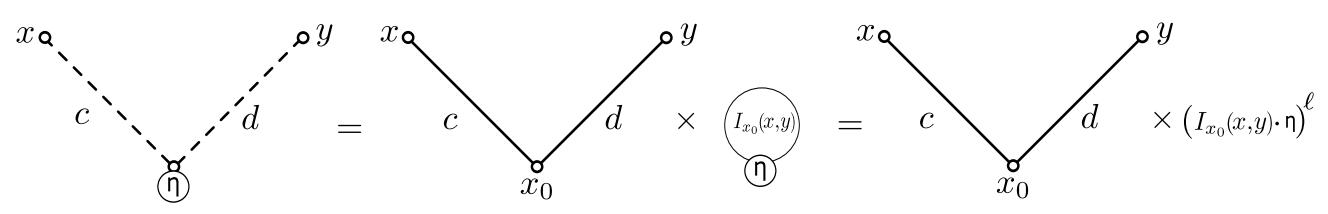

Figure 3. Solid lines stand for the usual Feynman diagram notation, that is the square distance of the type $(x-y)^{2}$ between the extremal points $x$ and $y$, to some power which is written adjacent to the line. Dashed line notation is illustrated in terms of product of solid lines and a tensor structure. The tensor $I_{x_{0}}^{\mu}(x, y)=\frac{x^{\mu}-x_{0}^{\mu}}{\left(x-x_{0}\right)^{2}}-\frac{y^{\mu}-x_{0}^{\mu}}{\left(y-x_{0}\right)^{2}}$ is obtained by a conformal inversion around the point $x_{0}$ of the vector $x^{\mu}-y^{\mu}$.
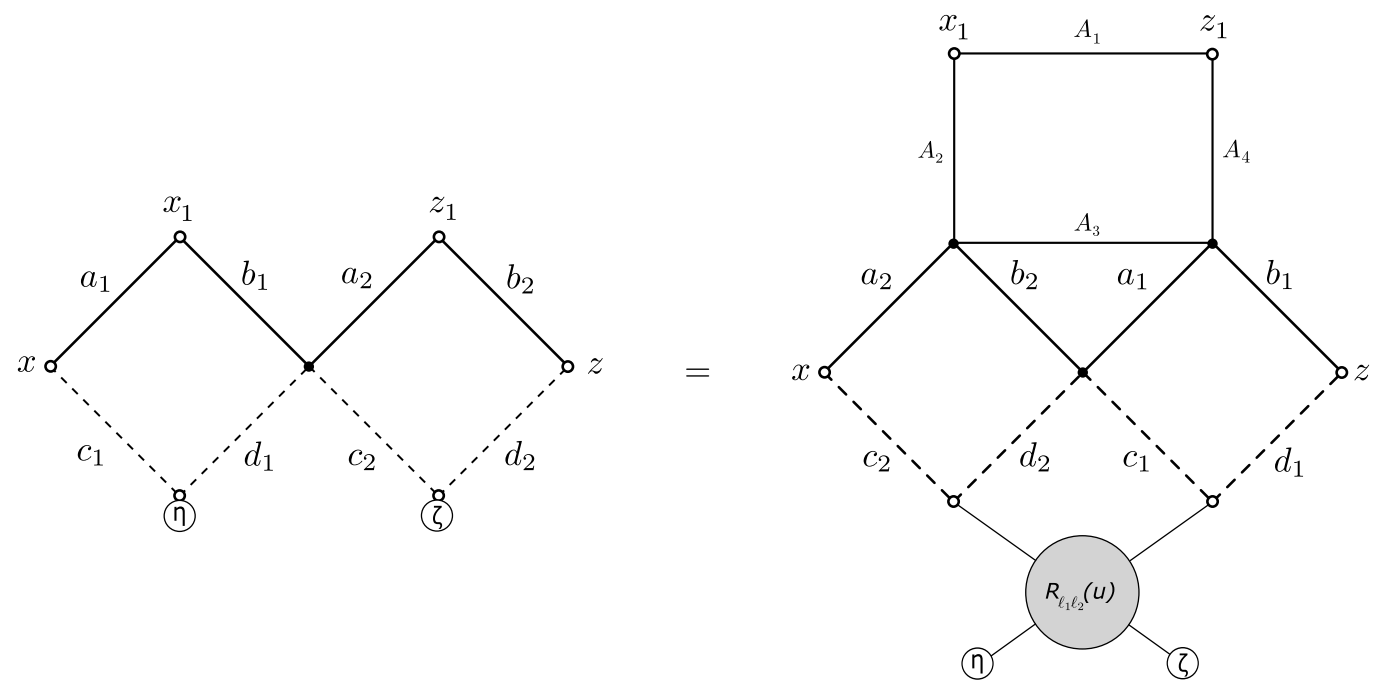

Figure 4. Graphical representation of the interchange relation. Black dots are integration points of the diagram, circles are external points. The powers $a_{j}, b_{j}, c_{j}, d_{j}$, for $j=1,2$, of the solid lines get exchanged between both sides of the identity.

and the powers of the square kernel in the right-hand side of figure 4 are

$$
\begin{aligned}
& A_{1}=\frac{d}{2}-1+\tilde{\beta}_{1}-\tilde{\beta}_{2}+\frac{l_{1}+l_{2}}{2}, A_{2}=\frac{d}{2}+\tilde{\beta}_{2}-\tilde{\beta}_{1}+\frac{l_{1}-l_{2}}{2}, \\
& A_{3}=1-\frac{d}{2}+\tilde{\beta}_{1}-\tilde{\beta}_{2}-\frac{l_{1}+l_{2}}{2}, A_{4}=\frac{d}{2}+\tilde{\beta}_{2}-\tilde{\beta}_{1}+\frac{l_{2}+l_{1}}{2} .
\end{aligned}
$$

The interchange relation is equivalent to an explicit integral representation for the matrix element of the operator $\mathbb{R}_{l_{1}, l_{2}}$, which we depict in figure 5 and reads

$$
\begin{aligned}
& {\left[\mathbb{R}_{l_{1}, l_{2}}(u) \zeta^{\otimes l_{1}} \otimes \eta^{\otimes l_{2}}\right] \cdot\left[\left(\frac{x}{x^{2}}-\frac{w}{w^{2}}\right)^{\otimes l_{1}} \otimes\left(\frac{y}{y^{2}}-\frac{w}{w^{2}}\right)^{\otimes l_{2}}\right]} \\
& =F_{l_{1}, l_{2}}(u) \frac{x^{2\left(\mathrm{i} u+\frac{l_{21}}{2}\right)} y^{2\left(\mathrm{i} u+\frac{l_{12}}{2}\right)}}{w^{2\left(\mathrm{i} u+\frac{l_{1}+l_{2}}{2}-1\right)}} \\
& \quad \times \int \frac{(z-w)^{2\left(\mathrm{i} u+\frac{l_{1}+l_{2}}{2}-1\right)}\left[\zeta \cdot\left(\frac{y}{y^{2}}-\frac{v}{v^{2}}\right)\right]^{l_{1}}\left[\eta \cdot\left(\frac{x}{x^{2}}-\frac{v}{v^{2}}\right)\right]^{l_{2}}}{(z-x)^{2\left(\mathrm{i} u+\frac{l_{21}}{2}\right)}(z-y)^{2\left(\mathrm{i} u+\frac{l_{12}}{2}\right)}(z-v)^{2\left(d-1+\frac{l_{1}+l_{2}}{2}-\mathrm{i} u\right)} v^{2\left(1-\frac{l_{1}+l_{2}}{2}+\mathrm{i} u\right)} \frac{\mathrm{d}^{d} z \mathrm{~d}^{d} v}{\pi^{d}},}
\end{aligned}
$$



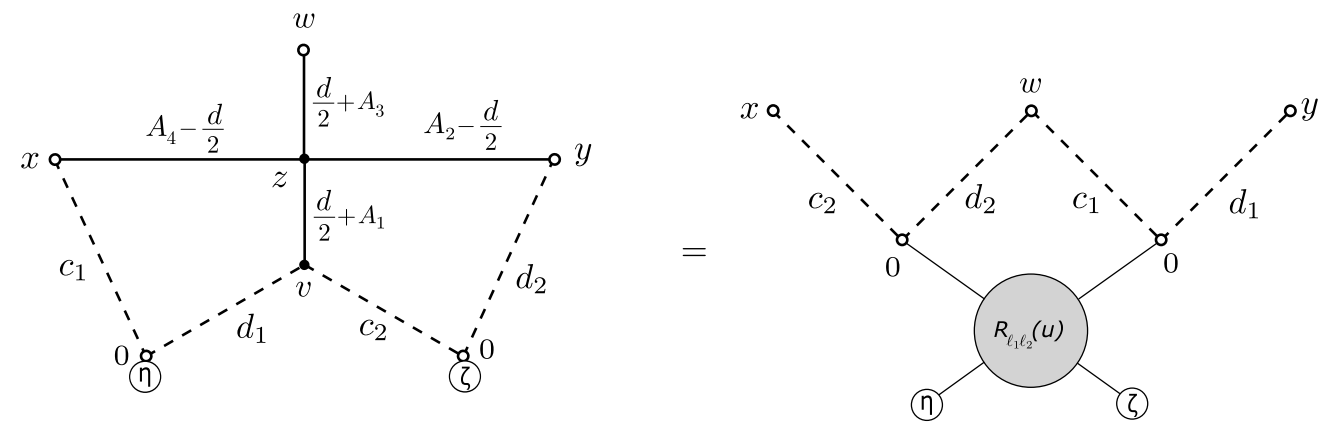

Figure 5. Integral representation of a matrix element of the fused R-matrix $\mathbb{R}_{l_{1}, l_{2}}(u)$, as encoded into the interchange relation.

where $\zeta^{2}=\eta^{2}=0$ but $x^{2}, y^{2}$ and $w^{2}$ are arbitrary, $l_{12}=-l_{21}=l_{1}-l_{2}$, and

$$
F_{l_{1}, l_{2}}(u)=\frac{\Gamma\left(1-\frac{l_{1}+l_{2}}{2}-\mathrm{i} u\right) \Gamma\left(1+\frac{l_{1}+l_{2}}{2}+\mathrm{i} u\right) \Gamma\left(d-1+\frac{l_{1}+l_{2}}{2}-\mathrm{i} u\right)}{\Gamma\left(1+\frac{l_{1}+l_{2}}{2}-\mathrm{i} u\right) \Gamma\left(\frac{d+l_{1}+l_{2}}{2}-1-\mathrm{i} u\right) \Gamma\left(1-\frac{l_{1}+l_{2}+d}{2}+\mathrm{i} u\right)} .
$$

We point out that in this expression, as well as in the other integral expressions that will appear in the rest of the paper, the exponents of the propagators are generic complex numbers due to presence of the spectral parameter $u$. We shall consider the generic situation $u \in \mathbb{C}$ and understand all similar integrals as an analytic continuation in $u$ from the convergence domain.

We show in figure 6 how to prove the interchange relation using the integral representation for the R-matrix. Since the steps can clearly be reordered, the two relations are actually equivalent. We shall prove in appendix (B) the equivalence of this integral expression and the expression (2.33).

In the following, we present the derivation of a few other integral representations for the matrix element (2.43). It is natural to perform an inversion of all external vectors $x, y, w$ and variables of integrations $z \mapsto \frac{z}{z^{2}}$ and $v \mapsto \frac{v}{v^{2}}$ in relation (2.43). After this transformation, one obtains

$$
\begin{aligned}
& {\left[\mathbb{R}_{l_{1}, l_{2}}(u) \zeta^{\otimes l_{1}} \otimes \eta^{\otimes l_{2}}\right] \cdot(x-w)^{\otimes l_{1}} \otimes(y-w)^{\otimes l_{2}}} \\
& =F_{l_{1}, l_{2}}(u) \int \frac{(z-w)^{2\left(\mathrm{i} u+\frac{l_{1}+l_{2}}{2}-1\right)}(\zeta \cdot(y-v))^{l_{1}}(\eta \cdot(x-v))^{l_{2}}}{(z-x)^{2\left(\mathrm{i} u+\frac{l_{21}}{2}\right)}(z-y)^{2\left(\mathrm{i} u+\frac{l_{12}}{2}\right)}(z-v)^{2\left(d-1+\frac{l_{1}+l_{2}}{2}-\mathrm{i} u\right)}} \frac{\mathrm{d}^{d} z \mathrm{~d}^{d} v}{\pi^{d}} .
\end{aligned}
$$

The integral representation in the right hand side shows manifestly the translation invariance. Thus, for simplicity, we may put $w=0$ without any loss of generality:

$$
\begin{aligned}
& {\left[\mathbb{R}_{l_{1}, l_{2}}(u) \zeta^{\otimes l_{1}} \otimes \eta^{\otimes l_{2}}\right] \cdot x^{\otimes l_{1}} \otimes y^{\otimes l_{2}}} \\
& =F_{l_{1}, l_{2}}(u) \int \frac{z^{2\left(\mathrm{i} u+\frac{l_{1}+l_{2}}{2}-1\right)}(\zeta \cdot(y-v))^{l_{1}}(\eta \cdot(x-v))^{l_{2}}}{(z-x)^{2\left(\mathrm{i} u+\frac{l_{21}}{2}\right)}(z-y)^{2\left(\mathrm{i} u+\frac{l_{12}}{2}\right)}(z-v)^{2\left(d-1+\frac{l_{1}+l_{2}}{2}-\mathrm{i} u\right)}} \frac{\mathrm{d}^{d} z \mathrm{~d}^{d} v}{\pi^{d}} .
\end{aligned}
$$

Despite its simplicity, this integral representation should be used with care because the integral over $v$ is ill defined. The origin of the problems is our naïve inversion of integration 

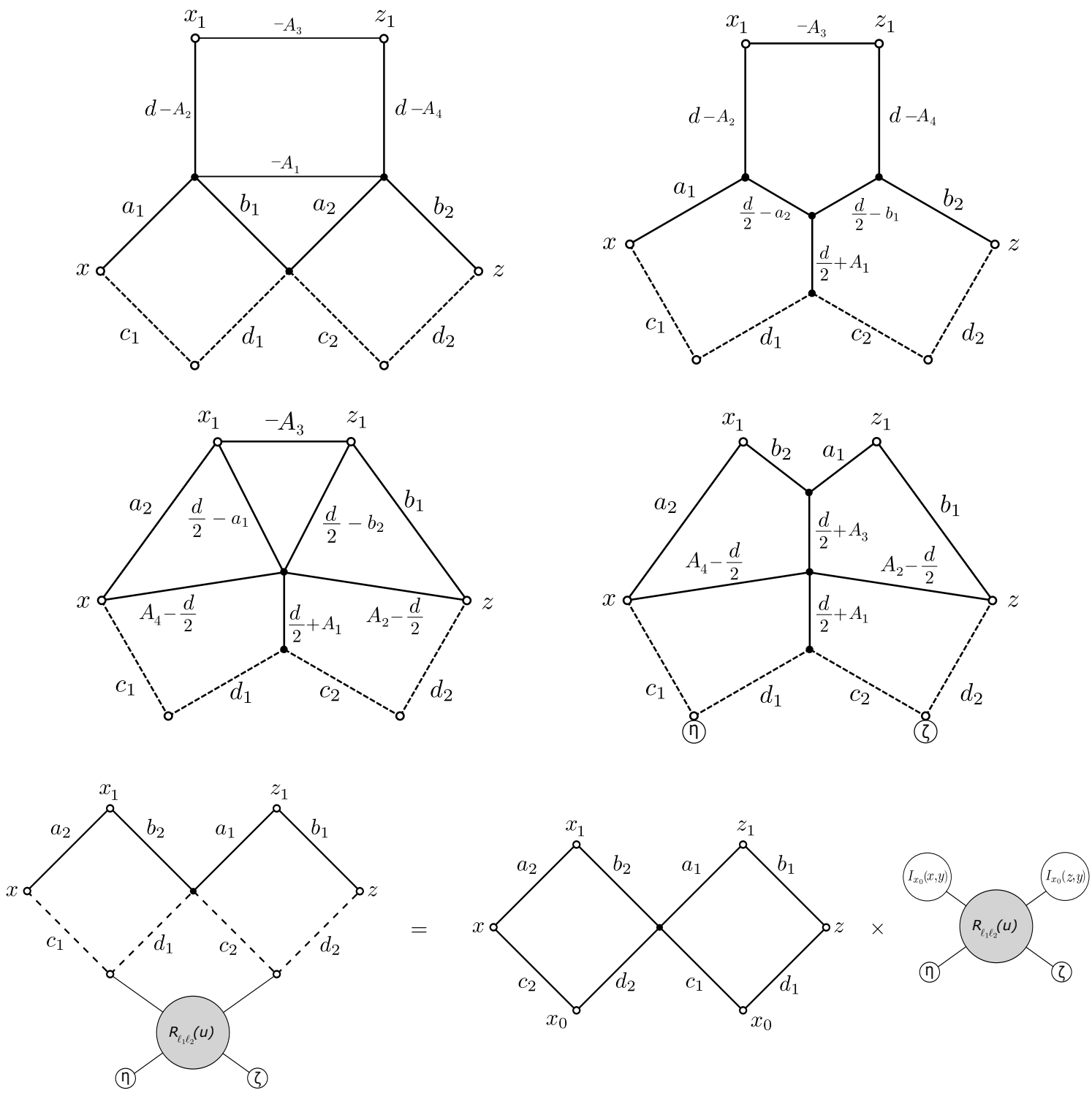

Figure 6. Top left: l.h.s. of the interchange relation after multiplication by the inverse of the square kernel. Top right: the central triangle $\left(-A_{1}, a_{2}, b_{1}\right)$ is transformed into a star integral. Middle left: result of star-triangle identity in the vertices $\left(a_{1}, d-A_{2}, d / 2-a_{2}\right)$ and $\left(b_{2}, d-A_{4}, d / 2-a_{1}\right)$. Middle right: the triangle $\left(-A_{3}, d / 2-a_{1}, d / 2-b_{2}\right)$ is transformed into a star integral. Bottom: result of the integration of the vertex $\left(d_{1}, c_{2}, d / 2+A_{1}\right)$. The tensors $I_{x_{0}}(x, y)^{\otimes l_{1}}$ and $I_{x_{0}}(z, y)^{\otimes l_{2}}$ get mixed by a non-trivial operator acting on $\mathbb{V}^{l_{1}} \otimes \mathbb{V}^{l_{2}}$, the matrix $\mathbb{R}_{l_{1} l_{2}}(u)$. 
variable $v \mapsto \frac{v}{v^{2}}$ in the initial expression, and we illustrate it on the much simpler example of the delta function (A.9)

$$
\int \frac{\mathrm{d}^{d} v}{v^{2 a}(z-v)^{2(d-a)}}=A_{0}(a) A_{0}(d-a) \pi^{d} \delta^{(d)}(z)
$$

If one naïvely performs an inversion $z \mapsto \frac{z}{z^{2}}$ and $v \mapsto \frac{v}{v^{2}}$, the equation becomes

$$
\int \frac{\mathrm{d}^{d} v}{(z-v)^{2(d-a)}}=A_{0}(a) A_{0}(d-a) \pi^{d} \frac{\delta^{(d)}\left(\frac{z}{z^{2}}\right)}{z^{2(d-a)}}
$$

where neither side is well defined. In order to obtain a well-defined expression for the R-matrix, we perform an inversion of all external vectors $x, y, w$ but not of the variables of integrations $z$ and $v$ in (2.43), this yields (here $w=0$ )

$$
\begin{aligned}
& {\left[\mathbb{R}_{l_{1}, l_{2}}(u) \zeta^{\otimes l_{1}} \otimes \eta^{\otimes l_{2}}\right] \cdot x^{\otimes l_{1}} \otimes y^{\otimes l_{2}}} \\
& =F_{l_{1}, l_{2}}(u) \int \frac{1}{\left(1-2 z \cdot x+z^{2} x^{2}\right)^{\mathrm{i} u+\frac{l_{21}}{2}}\left(1-2 z \cdot y+z^{2} y^{2}\right)^{\mathrm{i} u+\frac{l_{12}}{2}}} \\
& \quad \times \frac{\left(\zeta \cdot\left(y-\frac{v}{v^{2}}\right)\right)^{l_{1}}\left(\eta \cdot\left(x-\frac{v}{v^{2}}\right)\right)^{l_{2}}}{(z-v)^{2\left(d-1+\frac{l_{1}+l_{2}}{2}-\mathrm{i} u\right)} v^{2\left(1-\frac{l_{1}+l_{2}}{2}+\mathrm{i} u\right)} \frac{\mathrm{d}^{d} z \mathrm{~d}^{d} v}{\pi^{d}} .}
\end{aligned}
$$

The same representation can be obtained via the inversion $z \mapsto \frac{z}{z^{2}}$ and $v \mapsto \frac{v}{v^{2}}$ in relation (2.46). In fact, the integration of $v$ is reduced to the finite sum of derivatives of delta function, therefore the integration of $z$ can be performed easily, so that we can derive a closed expression for the integrals in r.h.s. of (2.49). The result reads (see appendix (B))

$$
\begin{aligned}
& \int \frac{\left(\zeta \cdot\left(y-\frac{v}{v^{2}}\right)\right)^{l_{1}}\left(\eta \cdot\left(x-\frac{v}{v^{2}}\right)\right)^{l_{2}}}{(z-v)^{2\left(d-1+\frac{l_{1}+l_{2}}{2}-\mathrm{i} u\right)} v^{2\left(1-\frac{l_{1}+l_{2}}{2}+\mathrm{i} u\right)}} \frac{\mathrm{d}^{d} v}{\pi^{\frac{d}{2}}} \\
& =A_{l_{1}, l_{2}}(u) \sum_{n, k, p} \frac{l_{1} ! l_{2} !(-1)^{k+p} 2^{-k-p-3 n}}{\left(l_{1}-k-n\right) !\left(l_{2}-p-n\right) ! k ! p ! n !} \\
& \frac{(\zeta \cdot \eta)^{n}(\zeta \cdot y)^{l_{1}-k-n}(\eta \cdot x)^{l_{2}-p-n}}{\left(1-\frac{l_{1}+l_{2}}{2}+\mathrm{i} u\right)_{k+p+2 n}\left(2-\frac{d}{2}-\frac{l_{1}+l_{2}}{2}+\mathrm{i} u\right)_{n}} \partial_{t}^{k} \partial_{s}^{p}\left(\partial_{z_{\mu}} \partial_{z_{\mu}}\right)^{n} \delta^{(d)}(z-t \zeta-s \eta)
\end{aligned}
$$

where we have to put $t=s=0$ after differentiation, and the explicit expression for $A_{l_{1}, l_{2}}(u)$ is given in B.6. For simplicity, we showed in the sum the summation indices only. The sum is finite and the range of summation is dictated by factorials in denominator: for each $0 \leqslant n \leqslant \min \left(l_{1}, l_{2}\right)$, we have $0 \leqslant k \leqslant l_{1}-n$ and $0 \leqslant p \leqslant l_{2}-n$. Now the integral in $z$ can 
be calculated due to the appearance of the delta function, and we finally obtain

$$
\begin{aligned}
& {\left[\mathbb{R}_{l_{1}, l_{2}}(u) \zeta^{\otimes l_{1}} \otimes \eta^{\otimes l_{2}}\right] \cdot x^{\otimes l_{1}} \otimes y^{\otimes l_{2}}} \\
& =\pi^{-\frac{d}{2}} F_{l_{1}, l_{2}}(u) A_{l_{1}, l_{2}}(u) \sum_{n, k, p} \frac{l_{1} ! l_{2} !(-1)^{k+p} 2^{-k-p-3 n}}{\left(l_{1}-k-n\right) !\left(l_{2}-p-n\right) ! k ! p ! n !} \\
& \\
& \quad \frac{(\zeta \cdot \eta)^{n}(\zeta \cdot y)^{l_{1}-k-n}(\eta \cdot x)^{l_{2}-p-n}}{\left(1-\frac{l_{1}+l_{2}}{2}+\mathrm{i} u\right)_{k+p+2 n}\left(2-\frac{d}{2}-\frac{l_{1}+l_{2}}{2}+\mathrm{i} u\right)_{n}} \partial_{t}^{k} \partial_{s}^{p} \\
& \left.\quad\left(\partial_{z_{\mu}} \partial_{z_{\mu}}\right)^{n} \frac{1}{\left(1-2 z \cdot x+z^{2} x^{2}\right)^{\mathrm{i} u+\frac{l_{21}}{2}}\left(1-2 z \cdot y+z^{2} y^{2}\right)^{\mathrm{i} u+\frac{l_{12}}{2}}}\right|_{z=t \zeta+s \eta} .
\end{aligned}
$$

The symbol $\left.\right|_{z=t \zeta+s \eta}$ means that we have to substitute $z=t \zeta+s \eta$ after all z-differentiation and then to put $t=s=0$ after all s- and t-differentiations.

It seems that the coincidence of expression (2.51) and (2.33) is far from obvious. The direct proof of their equivalence is very technical and is given in appendix (B). Note that the equivalence between (2.51) and (2.33) automatically guarantees the validity of the interchange relations in figure 4.

\subsection{Properties of the $\mathrm{R}$ matrices}

Even though the integral representation (2.46) should be used with care, it proves to be very useful. For example, it allows to reduce the derivation of some important properties of the R-matrix to a few simple standard steps: the integral chain rules (A.8) and (A.9) and the star-triangle relation (A.10).

Integral formula for null vectors. The fact that (2.51), or equivalently (2.46), is the same as (2.33) is proven in appendix (B). However, in the case $x^{2}=y^{2}=0$ everything is simpler and the integral over $z$ in (2.46) can be calculated explicitly using Symanzik's trick [49]: if the parameters $a_{1}, \ldots, a_{N}$ satisfy $\sum_{k=1}^{N} a_{k}=d$, then it holds that

$$
\int \prod_{k=1}^{N} \frac{\Gamma\left(a_{k}\right)}{\left(z-x_{k}\right)^{2 a_{k}}} \frac{\mathrm{d}^{d} z}{\pi^{\frac{d}{2}}}=\int_{\mathbb{R}_{+}^{N}} \frac{\mathrm{e}^{-\frac{\sum_{i, j} \alpha_{i} \alpha_{j}\left(x_{i}-x_{j}\right)^{2}}{\sum_{k=1}^{N} \gamma_{k} a_{k}}}}{\left(\sum_{k=1}^{N} \gamma_{k} a_{k}\right)^{\frac{d}{2}}} \prod_{k=1}^{N} \alpha_{k}^{a_{k}-1} \mathrm{~d} \alpha_{k},
$$

where the parameters $\gamma_{1}, \ldots, \gamma_{N}$ can be chosen arbitrarily as long as $\gamma_{k} \geqslant 0$, and they are not all zero. In our case, $N=4$ and we choose three of the parameters to be 0 whereas the last one is set to 1 , we thus obtain

$$
\begin{aligned}
& \int \frac{\Gamma\left(\mathrm{i} u+\frac{l_{21}}{2}\right) \Gamma\left(\mathrm{i} u+\frac{l_{12}}{2}\right) \Gamma\left(1-\frac{l_{1}+l_{2}}{2}-\mathrm{i} u\right) \Gamma\left(d-1+\frac{l_{1}+l_{2}}{2}-\mathrm{i} u\right)}{(z-x)^{2\left(\mathrm{i} u+\frac{l_{21}}{2}\right)}(z-y)^{2\left(\mathrm{i} u+\frac{l_{12}}{2}\right)} z^{2\left(1-\frac{l_{1}+l_{2}}{2}-\mathrm{i} u\right)}(z-v)^{2\left(d-1+\frac{l_{1}+l_{2}}{2}-\mathrm{i} u\right)}} \frac{\mathrm{d}^{d} z}{\pi^{\frac{d}{2}}} \\
& =\int_{\mathbb{R}_{+}^{4}} \alpha_{1}^{\mathrm{i} u+\frac{l_{21}}{2}-1} \alpha_{2}^{\mathrm{i} u+\frac{l_{12}}{2}-1} \alpha_{3}^{-\frac{l_{1}+l_{2}}{2}-\mathrm{i} u} \alpha_{4}^{\frac{d}{2}-2+\frac{l_{1}+l_{2}}{2}-\mathrm{i} u} \mathrm{e}^{-\frac{\alpha_{1} \alpha_{2}}{\alpha_{4}}(x-y)^{2}-\alpha_{1}(x-v)^{2}-\alpha_{2}(y-v)^{2}-\alpha_{3} v^{2}} \prod_{k=1}^{4} \mathrm{~d} \alpha_{k} \\
& =\frac{\Gamma\left(\frac{d}{2}+l_{1}-1\right) \Gamma\left(\frac{d}{2}+l_{2}-1\right) \Gamma\left(1-\frac{l_{1}+l_{2}}{2}-\mathrm{i} u\right) \Gamma\left(1-\frac{d+l_{1}+l_{2}}{2}+\mathrm{i} u\right)}{(y-v)^{2\left(\frac{d}{2}+l_{1}-1\right)}(x-v)^{2\left(\frac{d}{2}+l_{2}-1\right)} v^{2\left(1-\frac{l_{1}+l_{2}}{2}-\mathrm{i} u\right)}(x-y)^{2\left(1-\frac{d+l_{1}+l_{2}}{2}+\mathrm{i} u\right)} .}
\end{aligned}
$$


As a consequence, when $x$ and $y$ are null vectors, the formula (2.46) reduces to

$$
\begin{aligned}
& {\left[\mathbb{R}_{l_{1}, l_{2}}(u) \zeta^{\otimes l_{1}} \otimes \eta^{\otimes l_{2}}\right] \cdot\left(x^{\otimes l_{1}} \otimes y^{\otimes l_{2}}\right)} \\
& =\frac{\Gamma\left(\frac{d}{2}+l_{1}-1\right) \Gamma\left(\frac{d}{2}+l_{2}-1\right)\left(\mathrm{i} u+\frac{l_{1}+l_{2}}{2}\right) \Gamma\left(\mathrm{i} u-\frac{l_{1}+l_{2}}{2}\right)}{\Gamma\left(-\mathrm{i} u+\frac{d-2+l_{1}+l_{2}}{2}\right) \Gamma\left(\mathrm{i} u+\frac{l_{1}-l_{2}}{2}\right) \Gamma\left(\mathrm{i} u+\frac{l_{2}-l_{1}}{2}\right)} \\
& \quad \times(x-y)^{2\left(-\mathrm{i} u+\frac{d+l_{1}+l_{2}-2}{2}\right)} \int \frac{(\zeta \cdot(v-y))^{l_{1}}(\eta \cdot(v-x))^{l_{2}}}{v^{2\left(1-\mathrm{i} u-\frac{l_{1}+l_{2}}{2}\right.}(y-v)^{2\left(\frac{d}{2}+l_{1}-1\right)}(x-v)^{2\left(\frac{d}{2}+l_{2}-1\right)}} \frac{\mathrm{d}^{d} v}{\pi^{\frac{d}{2}}} .
\end{aligned}
$$

This integral is well-defined. We postpone to appendix (C) the direct check of the equivalence of this representation to the expression (2.3).

Derivative identity and mixing operator $\mathbb{O}_{l_{1}, l_{2}}$. For $\zeta$ and $\eta$ two null vectors, it holds that

$$
\begin{aligned}
(\zeta \cdot \nabla)^{l_{1}}(\eta \cdot \nabla)^{l_{2}} x^{2\left(\frac{l_{1}+l_{2}+2-d}{2}+\lambda\right)}= & \frac{\left(\frac{4-l_{1}-l_{2}-d}{2}+\lambda\right)_{l_{1}+l_{2}}}{\left(\frac{4-l_{1}-l_{2}-d}{2}-\lambda\right)_{l_{1}+l_{2}}}\left(x^{2}\right)^{2 \lambda} \\
& \times\left[\mathbb{R}_{l_{1}, l_{2}}(-\mathrm{i} \lambda) \zeta^{\otimes l_{1}} \otimes \eta^{\otimes l_{2}}\right] \cdot \nabla^{\otimes\left(l_{1}+l_{2}\right)} x^{2\left(\frac{l_{1}+l_{2}+2-d}{2}-\lambda\right)} .
\end{aligned}
$$

In order to prove this identity one needs to compute $y^{\otimes\left(l_{1}+l_{2}\right)} \cdot\left[\mathbb{R}_{l_{1}, l_{2}}(-\mathrm{i} \lambda) \zeta^{\otimes l_{1}} \otimes \eta^{\otimes l_{2}}\right]$ for arbitrary $y$. The details of calculation and the proof of the relation (2.55) are given in appendix (D).

Let us define an operator $\mathbb{O}_{l_{1}, l_{2}}(u): \mathbb{V}_{l_{1}} \otimes \mathbb{V}_{l_{2}} \rightarrow S^{l_{1}+l_{2}}\left(\mathbb{C}^{d}\right)$ that takes values in the space of symmetric tensors of rank $l_{1}+l_{2}$ in the following way:

$$
\begin{aligned}
& (\zeta \cdot \nabla)^{l_{1}}(\eta \cdot \nabla)^{l_{2}} x^{2\left(\frac{l_{1}+l_{2}+2-d}{2}+\mathrm{i} u\right)} \\
& =2^{l_{1}+l_{2}}\left(\frac{4-l_{1}-l_{2}-d}{2}+\mathrm{i} u\right)_{l_{1}+l_{2}} \frac{\left[\mathbb{O}_{l_{1}, l_{2}}(u) \zeta^{\otimes l_{1}} \otimes \eta^{\otimes l_{2}}\right] \cdot x^{\otimes\left(l_{1}+l_{2}\right)}}{x^{2\left(\frac{l_{1}+l_{2}+d-2}{2}-\mathrm{i} u\right)}},
\end{aligned}
$$

or, equivalently, using D.2,

$$
\left[\mathbb{O}_{l_{1}, l_{2}}(u) \zeta^{\otimes l_{1}} \otimes \eta^{\otimes l_{2}}\right] \cdot x^{\otimes\left(l_{1}+l_{2}\right)}=\sum_{p} \frac{l_{1} ! l_{2} !}{p !\left(l_{1}-p\right) !\left(l_{2}-p\right) !} \frac{\left(x^{2} \zeta \cdot \eta\right)^{p}(\zeta \cdot x)^{l_{1}-p}(\eta \cdot x)^{l_{2}-p}}{2^{p}\left(\frac{4-l_{1}-l_{2}-d}{2}+\mathrm{i} u\right)_{p}} .
$$

The property (2.55) we presented above is now written in a concise manner as

$$
\mathbb{O}_{l_{1}, l_{2}}(u)=\mathbb{O}_{l_{1}, l_{2}}(-u) \mathbb{R}_{l_{1}, l_{2}}(u) .
$$

The mixing operator $\mathbb{O}_{l_{1}, l_{2}}$ naturally arises in the generalisation of the chain relation (A.8):

$$
\begin{aligned}
& \int \frac{\mathrm{d}^{d} w}{\pi^{\frac{d}{2}}} \frac{C_{1}\left(\frac{w}{|w|}\right) C_{2}\left(\frac{w-x}{|w-x|}\right)}{w^{2 a}(w-x)^{2 b}} \\
& =A_{l_{1}}(a) A_{l_{2}}(b) A_{l_{1}+l_{2}}(d-a-b) \frac{\left[\mathbb{O}_{l_{1}, l_{2}}(\mathrm{i}(a+b+1-d)) C_{1} \otimes C_{2}\right] \cdot x^{\otimes\left(l_{1}+l_{2}\right)}}{x^{2\left(a+b+\frac{l_{1}+l_{2}-d}{2}\right)}}
\end{aligned}
$$




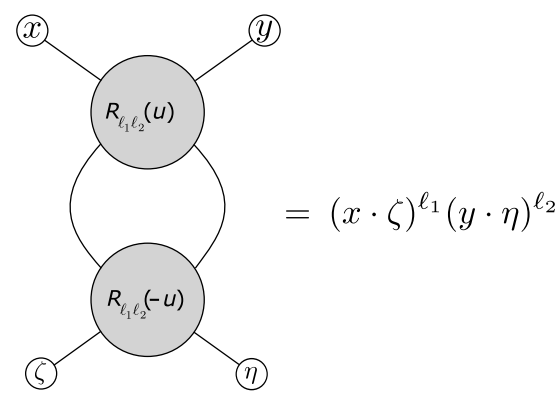

Figure 7. Property (2.61) in the notation for the fused $\mathbb{R}_{l_{1} l_{2}}(u)$ introduced in figure 2 .

and in the expression for the Basso-Dixon diagram (4.19).

Unitarity. The representation (2.3) clearly shows that the $\mathrm{R}$ matrices are symmetric and transform simply under complex conjugation:

$$
{ }^{t} \mathbb{R}_{l_{1}, l_{2}}=\mathbb{R}_{l_{1}, l_{2}}, \quad \mathbb{R}_{l_{1}, l_{2}}(u)^{*}=\mathbb{R}_{l_{1}, l_{2}}\left(-u^{*}\right) .
$$

From the integral representation (2.46), on the other hand, it is easy to see that the inverse is obtained by changing the sign of the spectral parameter:

$$
\mathbb{R}_{l_{1}, l_{2}}(u) \mathbb{R}_{l_{1}, l_{2}}(-u)=\operatorname{Id}_{l_{1}} \otimes \operatorname{Id}_{l_{2}}
$$

With the help of the two previous relations this amounts to saying that the R-matrix is unitary when $u$ is real. The proof of unitarity goes as follows:

$$
\begin{aligned}
& {\left[\mathbb{R}_{l_{1}, l_{2}}(-u) \mathbb{R}_{l_{1}, l_{2}}(u) \zeta^{\otimes l_{1}} \otimes \eta^{\otimes l_{2}}\right] \cdot\left(x^{\otimes l_{1}} \otimes y^{\otimes l_{2}}\right)} \\
& =F_{l_{1}, l_{2}}(-u) \int \frac{\left[\mathbb{R}_{l_{1}, l_{2}}(u) \zeta^{\otimes l_{1}} \otimes \eta^{\otimes l_{2}}\right] \cdot\left((y-v)^{\otimes l_{1}} \otimes(x-v)^{\otimes l_{2}}\right)}{(z-x)^{2\left(-\mathrm{i} u+\frac{l_{21}}{2}\right)}(z-y)^{2\left(-\mathrm{i} u+\frac{l_{12}}{2}\right)} z^{2\left(1-\frac{l_{1}+l_{2}}{2}+\mathrm{i} u\right)}(z-v)^{2\left(d-1+\frac{l_{1}+l_{2}}{2}+\mathrm{i} u\right)}} \frac{\mathrm{d}^{d} z \mathrm{~d}^{d} v}{\pi^{d}}
\end{aligned}
$$

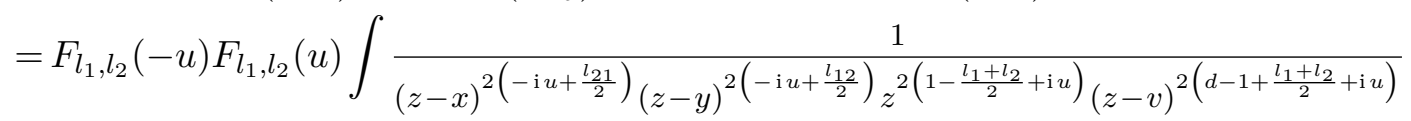

$$
\begin{aligned}
& \frac{\left(\zeta \cdot\left(x-v-v^{\prime}\right)\right)^{l_{1}}\left(\eta \cdot\left(y-v-v^{\prime}\right)\right)^{l_{2}}}{\left.\left(z^{\prime}+v-y\right)^{2\left(\mathrm{i} u+\frac{l_{21}}{2}\right.}\right)\left(z^{\prime}+v-x\right)^{2\left(-\mathrm{i} u+\frac{l_{12}}{2}\right)} z^{\prime 2\left(1-\frac{l_{1}+l_{2}}{2}-\mathrm{i} u\right)}\left(z^{\prime}-v^{\prime}\right)^{2\left(d-1+\frac{l_{1}+l_{2}}{2}-\mathrm{i} u\right)}} \frac{\mathrm{d}^{d} z^{\prime} \mathrm{d}^{d} v^{\prime} \mathrm{d}^{d} z \mathrm{~d}^{d} v}{\pi^{2 d}} .
\end{aligned}
$$

After the natural change of variables $v^{\prime} \rightarrow v^{\prime}-v$ and $z^{\prime} \rightarrow z^{\prime}-v$, it is possible to integrate over $v$ explicitly using (A.9). One obtains $\delta^{(d)}\left(z-z^{\prime}\right)$, and the integration over $z^{\prime}$ reduces 
the whole expression to another integral of the type (A.9)

$$
\begin{aligned}
& F_{l_{1}, l_{2}}(-u) F_{l_{1}, l_{2}}(u) \int \frac{1}{(z-x)^{2\left(-\mathrm{i} u+\frac{l_{21}}{2}\right)}(z-y)^{2\left(-\mathrm{i} u+\frac{l_{12}}{2}\right)} z^{2\left(1-\frac{l_{1}+l_{2}}{2}+\mathrm{i} u\right)}(z-v)^{2\left(d-1+\frac{l_{1}+l_{2}}{2}+\mathrm{i} u\right)}} \\
& \frac{\left(\zeta \cdot\left(x-v^{\prime}\right)\right)^{l_{1}}\left(\eta \cdot\left(y-v^{\prime}\right)\right)^{l_{2}}}{\left(z^{\prime}-y\right)^{2\left(\mathrm{i} u+\frac{l_{21}}{2}\right)}\left(z^{\prime}-x\right)^{2\left(-\mathrm{i} u+\frac{l_{12}}{2}\right)}\left(z^{\prime}-v\right)^{2\left(1-\frac{l_{1}+l_{2}}{2}-\mathrm{i} u\right)}\left(z^{\prime}-v^{\prime}\right)^{2\left(d-1+\frac{l_{1}+l_{2}}{2}-\mathrm{i} u\right)}} \frac{\mathrm{d}^{d} z^{\prime} \mathrm{d}^{d} v^{\prime} \mathrm{d}^{d} z \mathrm{~d}^{d} v}{\pi^{2 d}}
\end{aligned}
$$

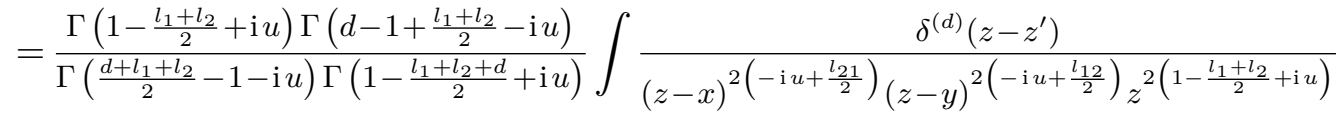

$$
\begin{aligned}
& \times \frac{\left(\zeta \cdot\left(x-v^{\prime}\right)\right)^{l_{1}}\left(\eta \cdot\left(y-v^{\prime}\right)\right)^{l_{2}}}{\left(z^{\prime}-y\right)^{2\left(\mathrm{i} u+\frac{l_{21}}{2}\right)}\left(z^{\prime}-x\right)^{2\left(-\mathrm{i} u+\frac{l_{12}}{2}\right)}\left(z^{\prime}-v^{\prime}\right)^{2\left(d-1+\frac{l_{1}+l_{2}}{2}-\mathrm{i} u\right)}} \frac{\mathrm{d}^{d} z^{\prime} \mathrm{d}^{d} v^{\prime} \mathrm{d}^{d} z}{\pi^{d}} \\
& =\frac{\Gamma\left(1-\frac{l_{1}+l_{2}}{2}+\mathrm{i} u\right) \Gamma\left(d-1+\frac{l_{1}+l_{2}}{2}-\mathrm{i} u\right)}{\Gamma\left(\frac{d+l_{1}+l_{2}}{2}-1-\mathrm{i} u\right) \Gamma\left(1-\frac{l_{1}+l_{2}+d}{2}+\mathrm{i} u\right)} \int \frac{\left(\zeta \cdot\left(x-v^{\prime}\right)\right)^{l_{1}}\left(\eta \cdot\left(y-v^{\prime}\right)\right)^{l_{2}}}{z^{2\left(1-\frac{l_{1}+l_{2}}{2}+\mathrm{i} u\right)}\left(z-v^{\prime}\right)^{2\left(d-1+\frac{l_{1}+l_{2}}{2}-\mathrm{i} u\right)}} \frac{\mathrm{d}^{d} v^{\prime} \mathrm{d}^{d} z}{\pi^{d}} \\
& =\int\left(\zeta \cdot\left(x-v^{\prime}\right)\right)^{l_{1}}\left(\eta \cdot\left(y-v^{\prime}\right)\right)^{l_{2}} \delta^{(d)}\left(v^{\prime}\right) \mathrm{d}^{d} v^{\prime}=(\zeta \cdot x)^{l_{1}}(\eta \cdot y)^{l_{2}} \text {. }
\end{aligned}
$$

Crossing symmetry. From the explicit representation (2.3) of the R-matrix, one immediately deduces the following crossing property:

$$
t_{2} \mathbb{R}_{l_{1}, l_{2}}\left(\mathrm{i} \frac{2-d}{2}-u\right)=\frac{\left(-\mathrm{i} u+\frac{d+l_{2}-l_{1}-2}{2}\right)_{l_{1}}\left(\mathrm{i} u-\frac{l_{1}+l_{2}}{2}\right)_{l_{1}}}{\left(-\mathrm{i} u+\frac{d-l_{1}-l_{2}-2}{2}\right)_{l_{1}}\left(\mathrm{i} u+\frac{l_{2}-l_{1}}{2}\right)_{l_{1}}} \mathbb{R}_{l_{1}, l_{2}}(u),
$$

where $t_{2}$ denotes transposition in $\mathbb{V}_{2}$ only.

Yang-Baxter relation. The fusion procedure being a way to construct new solutions of the Yang-Baxter relation, we know that the expression (2.3) satisfies it. It is however also possible to show it directly for the integral representation as we now explain. We want to show that for arbitrary null vectors $\zeta, \eta$, and $\theta$ we have

$$
\begin{aligned}
& \mathbb{R}_{l_{1}, l_{2}}(\lambda) \mathbb{R}_{l_{1}, l_{3}}(\lambda+\mu) \mathbb{R}_{l_{2}, l_{3}}(\mu) \zeta^{\otimes l_{1}} \otimes \eta^{\otimes l_{2}} \otimes \theta^{\otimes l_{3}} \\
& =\mathbb{R}_{l_{2}, l_{3}}(\mu) \mathbb{R}_{l_{1}, l_{3}}(\lambda+\mu) \mathbb{R}_{l_{1}, l_{2}}(\lambda) \zeta^{\otimes l_{1}} \otimes \eta^{\otimes l_{2}} \otimes \theta^{\otimes l_{3}}
\end{aligned}
$$

It suffices to verify that the scalar product with any vector of the form $x^{\otimes l_{1}} \otimes y^{\otimes l_{2}} \otimes$ $z^{\otimes l_{3}}$, for $x, y$, and $z$ real, is the same for both sides. After taking the scalar product and using the integral representation (without writing the scalar prefactors $F_{l_{i}, l_{j}}$ ), 

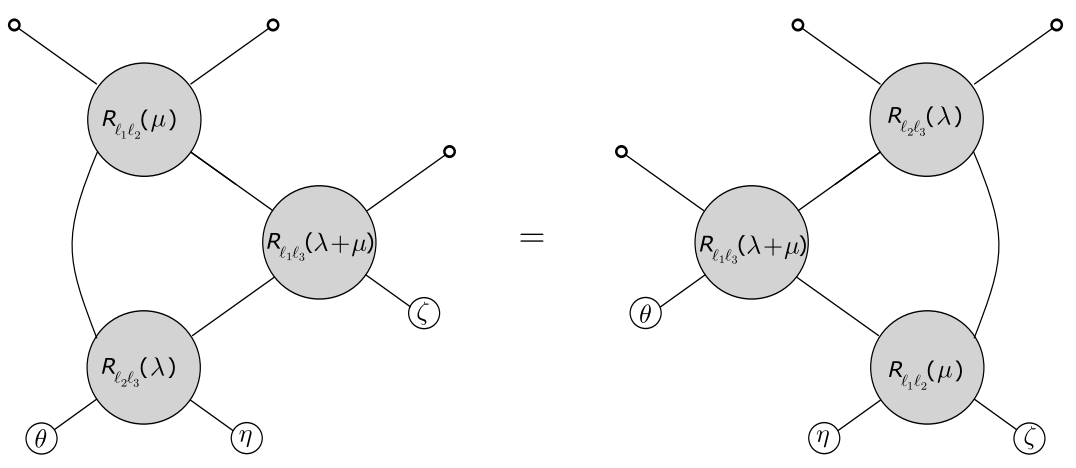

Figure 8. Property (2.63) in the notation for the fused $\mathbb{R}_{l_{1} l_{2}}(u)$ introduced in figure 2 .

the left-hand side becomes

$$
\begin{aligned}
& {\left[\mathbb{R}_{l_{1}, l_{2}}(\lambda) \mathbb{R}_{l_{1}, l_{3}}(\lambda+\mu) \mathbb{R}_{l_{2}, l_{3}}(\mu) \zeta^{\otimes l_{1}} \otimes \eta^{\otimes l_{2}} \otimes \theta^{\otimes l_{3}}\right] \cdot x^{\otimes l_{1}} \otimes y^{\otimes l_{2}} \otimes z^{\otimes l_{3}}} \\
& \propto \int \frac{\left[\mathbb{R}_{l_{1}, l_{3}}(\lambda+\mu) \mathbb{R}_{l_{2}, l_{3}}(\mu) \zeta^{\otimes l_{1}} \otimes \eta^{\otimes l_{2}} \otimes \theta^{\otimes l_{3}}\right] \cdot(y-v)^{\otimes l_{1}} \otimes(x-v)^{\otimes l_{2}} \otimes z^{\otimes l_{3}}}{(w-x)^{2\left(\mathrm{i} \lambda+\frac{l_{21}}{2}\right)}(w-y)^{2\left(\mathrm{i} \lambda+\frac{l_{12}}{2}\right)} w^{2\left(1-\frac{l_{1}+l_{2}}{2}-\mathrm{i} \lambda\right)}(w-v)^{2\left(d-1+\frac{l_{1}+l_{2}}{2}-\mathrm{i} \lambda\right)}} \frac{\mathrm{d}^{d} w \mathrm{~d}^{d} v}{\pi^{d}} \\
& \propto \int \frac{\pi^{-d} \mathrm{~d}^{d} w \mathrm{~d}^{d} v}{(w-x)^{2\left(\mathrm{i} \lambda+\frac{l_{21}}{2}\right)}(w-y)^{2\left(\mathrm{i} \lambda+\frac{l_{12}}{2}\right)} w^{2\left(1-\frac{l_{1}+l_{2}}{2}-\mathrm{i} \lambda\right)}(w-v)^{2\left(d-1+\frac{l_{1}+l_{2}}{2}-\mathrm{i} \lambda\right)}} \\
& \times \frac{\left[\mathbb{R}_{l_{2}, l_{3}}(\mu) \zeta^{\otimes l_{1}} \otimes \eta^{\otimes l_{2}} \otimes \theta^{\otimes l_{3}}\right] \cdot\left(z-v^{\prime}\right)^{\otimes l_{1}} \otimes(x-v)^{\otimes l_{2}} \otimes\left(y-v-v^{\prime}\right)^{\otimes l_{3}}}{\left(w^{\prime}-y+v\right)^{2\left(\mathrm{i} \lambda+\mathrm{i} \mu+\frac{l_{31}}{2}\right)}\left(w^{\prime}-z\right)^{2\left(\mathrm{i} \lambda+\mathrm{i} \mu+\frac{l_{13}}{2}\right)} w^{\prime 2\left(1-\frac{l_{1}+l_{3}}{2}-\mathrm{i} \lambda-\mathrm{i} \mu\right)}\left(w^{\prime}-v^{\prime}\right)^{2\left(d-1+\frac{l_{1}+l_{3}}{2}-\mathrm{i} \lambda-\mathrm{i} \mu\right)}} \frac{\mathrm{d}^{d} w^{\prime} \mathrm{d}^{d} v^{\prime}}{\pi^{d}} \\
& \propto \int \frac{\pi^{-d} \mathrm{~d}^{d} w \mathrm{~d}^{d} v}{(w-x)^{2\left(\mathrm{i} \lambda+\frac{l_{21}}{2}\right)}(w-y)^{2\left(\mathrm{i} \lambda+\frac{l_{12}}{2}\right)} w^{2\left(1-\frac{l_{1}+l_{2}}{2}-\mathrm{i} \lambda\right)}(w-v)^{2\left(d-1+\frac{l_{1}+l_{2}}{2}-\mathrm{i} \lambda\right)}} \\
& \times \frac{\pi^{-d} \mathrm{~d}^{d} w^{\prime} \mathrm{d}^{d} v^{\prime}}{\left(w^{\prime}-y+v\right)^{2\left(\mathrm{i} \lambda+\mathrm{i} \mu+\frac{l_{31}}{2}\right)}\left(w^{\prime}-z\right)^{2\left(\mathrm{i} \lambda+\mathrm{i} \mu+\frac{l_{13}}{2}\right)} w^{\prime 2\left(1-\frac{l_{1}+l_{3}}{2}-\mathrm{i} \lambda-\mathrm{i} \mu\right)}\left(w^{\prime}-v^{\prime}\right)^{2\left(d-1+\frac{l_{1}+l_{3}}{2}-\mathrm{i} \lambda-\mathrm{i} \mu\right)}} \\
& \times \frac{\left(\zeta \cdot\left(z-v^{\prime}\right)\right)^{l_{1}}\left(\eta \cdot\left(y-v-v^{\prime}-v^{\prime \prime}\right)\right)^{l_{2}}\left(\theta \cdot\left(x-v-v^{\prime \prime}\right)\right)^{l_{3}} \pi^{-d} \mathrm{~d}^{d} w^{\prime \prime} \mathrm{d}^{d} v^{\prime \prime}}{\left(w^{\prime \prime}+v-x\right)^{2\left(\mathrm{i} \mu+\frac{l_{32}}{2}\right)}\left(w^{\prime \prime}+v+v^{\prime}-y\right)^{2\left(\mathrm{i} \mu+\frac{l_{23}}{2}\right)} w^{\prime \prime 2\left(1-\frac{l_{2}+l_{3}}{2}-\mathrm{i} \mu\right)}\left(w^{\prime \prime}-v^{\prime \prime}\right)^{2\left(d-1+\frac{l_{2}+l_{3}}{2}-\mathrm{i} \mu\right)}} \\
& \propto \int \frac{\pi^{-d} \mathrm{~d}^{d} w \mathrm{~d}^{d} v}{(w-x)^{2\left(\mathrm{i} \lambda+\frac{l_{21}}{2}\right)}(w-y)^{2\left(\mathrm{i} \lambda+\frac{l_{12}}{2}\right)} w^{2\left(1-\frac{l_{1}+l_{2}}{2}-\mathrm{i} \lambda\right)}(w-v)^{2\left(d-1+\frac{l_{1}+l_{2}}{2}-\mathrm{i} \lambda\right)}} \\
& \times \frac{\pi^{-d} \mathrm{~d}^{d} w^{\prime} \mathrm{d}^{d} v^{\prime}}{\left(w^{\prime}-y+v\right)^{2\left(\mathrm{i} \lambda+\mathrm{i} \mu+\frac{l_{31}}{2}\right)}\left(w^{\prime}-z\right)^{2\left(\mathrm{i} \lambda+\mathrm{i} \mu+\frac{l_{13}}{2}\right)} w^{2\left(1-\frac{l_{1}+l_{3}}{2}-\mathrm{i} \lambda-\mathrm{i} \mu\right)}\left(w^{\prime}-v^{\prime}\right)^{2\left(d-1+\frac{l_{1}+l_{3}}{2}-\mathrm{i} \lambda-\mathrm{i} \mu\right)}} \\
& \times \frac{\left(\zeta \cdot\left(z-v^{\prime}\right)\right)^{l_{1}}\left(\eta \cdot\left(y-v^{\prime}-v^{\prime \prime}\right)\right)^{l_{2}}\left(\theta \cdot\left(x-v^{\prime \prime}\right)\right)^{l_{3}} \pi^{-d} \mathrm{~d}^{d} w^{\prime \prime} \mathrm{d}^{d} v^{\prime \prime}}{\left(w^{\prime \prime}-x\right)^{2\left(\mathrm{i} \mu+\frac{l_{32}}{2}\right)}\left(w^{\prime \prime}+v^{\prime}-y\right)^{2\left(\mathrm{i} \mu+\frac{l_{23}}{2}\right)}\left(w^{\prime \prime}-v\right)^{2\left(1-\frac{l_{2}+l_{3}}{2}-\mathrm{i} \mu\right)}\left(w^{\prime \prime}-v^{\prime \prime}\right)^{2\left(d-1+\frac{l_{2}+l_{3}}{2}-\mathrm{i} \mu\right)}} .
\end{aligned}
$$

At the last step, we have simply performed the change of variables $\left(w^{\prime \prime}, v^{\prime \prime}\right) \mapsto\left(w^{\prime \prime}-v, v^{\prime \prime}-v\right)$ so that now the integral over $v$ is computed by a simple application of the star-triangle identity (A.10). At the same time, we find it convenient to define $\tilde{z}=z-y$ and to perform 

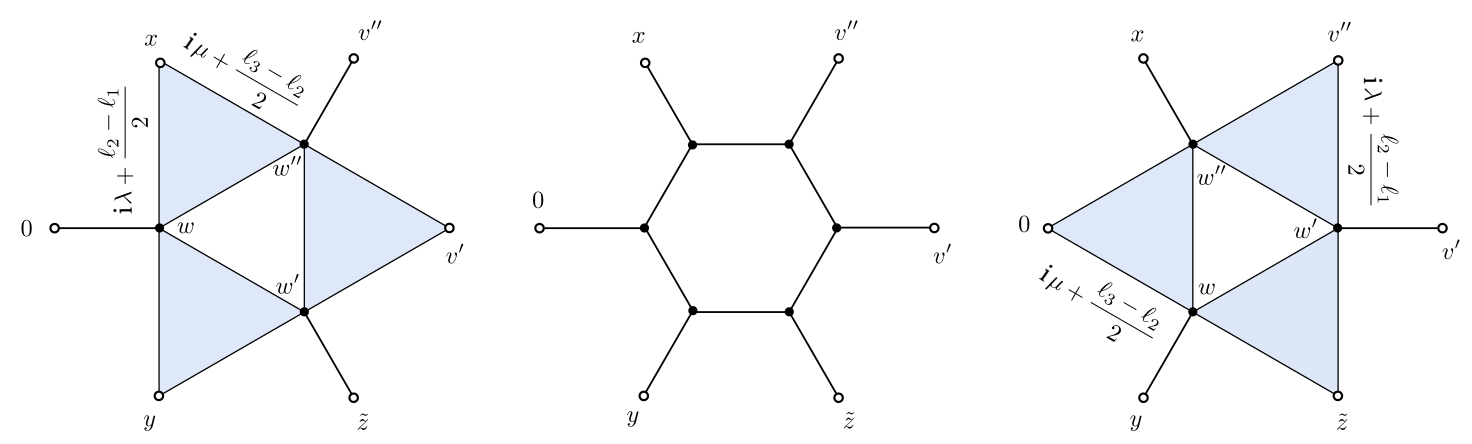

Figure 9. The kernels depicted on the left and on the right are related respectively to the l.h.s. and r.h.s. of the Yang-Baxter equation (2.63) in the integral representation for the R-matrix elements (2.3), and the equality of the two kernels is equivalent to the YBE. Both kernels are shown to be proportional to the one in the middle, via star-triangle identity applied to the blue triangles. Keeping track of the proportionality constants shows that l.h.s.=r.h.s.

the change of variables $\left(w^{\prime}, v^{\prime}\right) \mapsto\left(y-w^{\prime}, y-v^{\prime}\right)$, so that we obtain

$$
\begin{aligned}
& {\left[\mathbb{R}_{l_{1}, l_{2}}(\lambda) \mathbb{R}_{l_{1}, l_{3}}(\lambda+\mu) \mathbb{R}_{l_{2}, l_{3}}(\mu) \zeta^{\otimes l_{1}} \otimes \eta^{\otimes l_{2}} \otimes \theta^{\otimes l_{3}}\right] \cdot x^{\otimes l_{1}} \otimes y^{\otimes l_{2}} \otimes z^{\otimes l_{3}}} \\
& = \\
& \quad F_{l_{1}, l_{2}}(\lambda) F_{l_{1}, l_{3}}(\lambda+\mu) F_{l_{2}, l_{3}}(\mu) A_{0}\left(d-1+\frac{l_{1}+l_{2}}{2}-\mathrm{i} \lambda\right) A_{0}\left(\mathrm{i} \lambda+\mathrm{i} \mu+\frac{l_{31}}{2}\right) A_{0}\left(1-\frac{l_{2}+l_{3}}{2}-\mathrm{i} \mu\right) \\
& \quad \times \int \frac{\pi^{-\frac{d}{2}} \mathrm{~d}^{d} w}{\left.(w-x)^{2\left(\mathrm{i} \lambda+\frac{l_{2}-l_{1}}{2}\right.}\right)(w-y)^{2\left(\mathrm{i} \lambda+\frac{l_{1}-l_{2}}{2}\right)} w^{2\left(1-\frac{l_{1}+l_{2}}{2}-\mathrm{i} \lambda\right)}\left(w^{\prime}-w^{\prime \prime}\right)^{2\left(1-\frac{d+l_{1}+l_{2}}{2}+\mathrm{i} \lambda\right)}} \\
& \quad \times \frac{\pi^{-d} \mathrm{~d}^{d} w^{\prime} \mathrm{d}^{d} v^{\prime}}{\left(w^{\prime \prime}-w\right)^{2\left(\frac{d+l_{1}-l_{3}}{2}-\mathrm{i} \lambda-\mathrm{i} \mu\right)}\left(w^{\prime}-\tilde{z}\right)^{2\left(\mathrm{i} \lambda+\mathrm{i} \mu+\frac{l_{1}-l_{3}}{2}\right)}\left(w^{\prime}-y\right)^{2\left(1-\frac{l_{1}+l_{3}}{2}-\mathrm{i} \lambda-\mathrm{i} \mu\right)}\left(w^{\prime}-v^{\prime}\right)^{2\left(d-1+\frac{l_{1}+l_{3}}{2}-\mathrm{i} \lambda-\mathrm{i} \mu\right)}} \\
& \quad \times \frac{\left(\zeta \cdot\left(\tilde{z}+v^{\prime}\right)\right)^{l_{1}}\left(\eta \cdot\left(v^{\prime}-v^{\prime \prime}\right)\right)^{l_{2}}\left(\theta \cdot\left(x-v^{\prime \prime}\right)\right)^{l_{3}} \pi^{-d} \mathrm{~d}^{d} w^{\prime \prime} \mathrm{d}^{d} v^{\prime \prime}}{\left(w^{\prime \prime}-x\right)^{2\left(\mathrm{i} \mu+\frac{l_{3}-l_{2}}{2}\right)}\left(w^{\prime \prime}-v^{\prime}\right)^{2\left(\mathrm{i} \mu+\frac{l_{2}-l l_{3}}{2}\right)}\left(w-w^{\prime}\right)^{2\left(\frac{d+l_{2}+l_{3}}{2}-1+\mathrm{i} \mu\right)}\left(w^{\prime \prime}-v^{\prime \prime}\right)^{2\left(d-1+\frac{l_{2}+l_{3}}{2}-\mathrm{i} \mu\right)}} .
\end{aligned}
$$

Similar manipulations for the right-hand side of the Yang-Baxter relation give

$$
\begin{aligned}
& {\left[\mathbb{R}_{l_{2}, l_{3}}(\mu) \mathbb{R}_{l_{1}, l_{3}}(\lambda+\mu) \mathbb{R}_{l_{1}, l_{2}}(\lambda) \zeta^{\otimes l_{1}} \otimes \eta^{\otimes l_{2}} \otimes \theta^{\otimes l_{3}}\right] \cdot x^{\otimes l_{1}} \otimes y^{\otimes l_{2}} \otimes z^{\otimes l_{3}}} \\
& =F_{l_{1}, l_{2}}(\lambda) F_{l_{1}, l_{3}}(\lambda+\mu) F_{l_{2}, l_{3}}(\mu) A_{0}\left(d-1+\frac{l_{2}+l_{3}}{2}-\mathrm{i} \mu\right) A_{0}\left(\mathrm{i} \lambda+\mathrm{i} \mu+\frac{l_{13}}{2}\right) A_{0}\left(1-\frac{l_{1}+l_{2}}{2}-\mathrm{i} \lambda\right) \\
& \times \int \frac{\pi^{-\frac{d}{2}} \mathrm{~d}^{d} w}{w^{2\left(\mathrm{i} \mu+\frac{l_{3}-l_{2}}{2}\right)}(w-\tilde{z})^{2\left(\mathrm{i} \mu+\frac{l_{2}-l_{3}}{2}\right)}(w-y)^{2\left(1-\frac{l_{2}+l_{3}}{2}-\mathrm{i} \mu\right)}\left(w^{\prime}-w^{\prime \prime}\right)^{2\left(1-\frac{d+l_{2}+l_{3}}{2}+\mathrm{i} \mu\right)}} \\
& \times \frac{\pi^{-d} \mathrm{~d}^{d} w^{\prime \prime} \mathrm{d}^{d} v^{\prime \prime}}{\left(w^{\prime \prime}-x\right)^{2\left(\mathrm{i} \lambda+\mathrm{i} \mu+\frac{l_{3}-l_{1}}{2}\right)}\left(w-w^{\prime}\right)^{2\left(\frac{d+l_{3}-l_{1}}{2}-\mathrm{i} \lambda-\mathrm{i} \mu\right)} w^{\prime \prime 2\left(1-\frac{l_{1}+l_{3}}{2}-\mathrm{i} \lambda-\mathrm{i} \mu\right)}\left(w^{\prime \prime}-v^{\prime \prime}\right)^{2\left(d-1+\frac{l_{1}+l_{3}}{2}-\mathrm{i} \lambda-\mathrm{i} \mu\right)}} \\
& \times \frac{\left(\zeta \cdot\left(\tilde{z}+v^{\prime}\right)\right)^{l_{1}}\left(\eta \cdot\left(v^{\prime}-v^{\prime \prime}\right)\right)^{l_{2}}\left(\theta \cdot\left(x-v^{\prime \prime}\right)\right)^{l_{3}} \pi^{-d} \mathrm{~d}^{d} w^{\prime} \mathrm{d}^{d} v^{\prime}}{\left(w^{\prime}-v^{\prime \prime}\right)^{2\left(\mathrm{i} \lambda+\frac{l_{2}-l_{1}}{2}\right)}\left(w^{\prime}-\tilde{z}\right)^{2\left(\mathrm{i} \lambda+\frac{l_{1}-l_{2}}{2}\right)}\left(w-w^{\prime \prime}\right)^{2\left(\frac{d+l_{1}+l_{2}}{2}-1+\mathrm{i} \lambda\right)}\left(w^{\prime}-v^{\prime}\right)^{2\left(d-1+\frac{l_{1}+l_{2}}{2}-\mathrm{i} \lambda\right)} .}
\end{aligned}
$$

Notice now that the numerators in the integrands of the last two formulas are the same, and that these do not involve $w, w^{\prime}$, or $w^{\prime \prime}$. Consequently, if we can prove that the integrals over these three variables coincide, then we are done. This is actually a straightforward application of the star-triangle identity (A.10), as depicted in figure 9. 

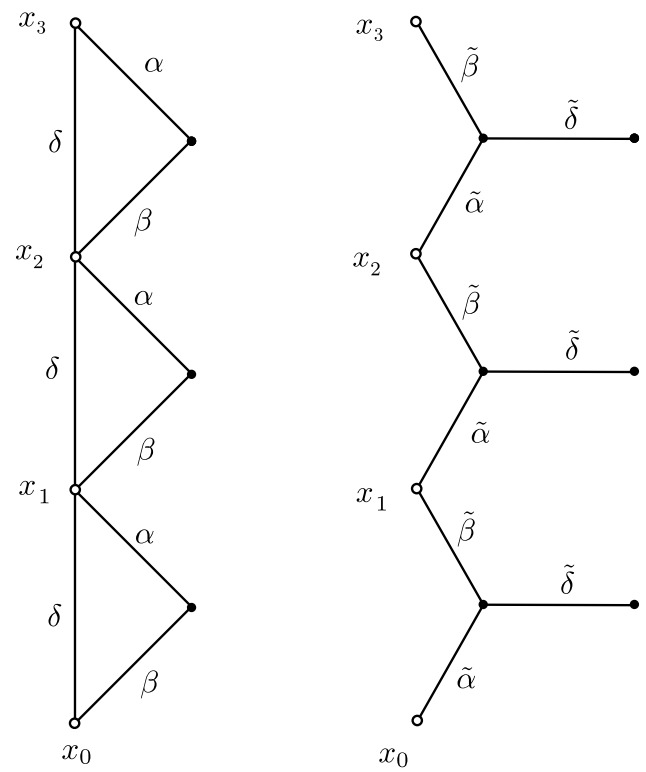

Figure 10. Transfer-matrix operator $\mathbf{Q}_{3}(u)$ as an integral operator with the kernel represented in Feynman diagram notation. The two equivalent forms are related by star-triangle identity.

\section{Diagonalisation of graph-building operators}

\subsection{Construction of the eigenvectors}

In this section, we will work with the choice $\delta \in \mathrm{i} \mathbb{R}$, corresponding to a representation of the unitary principal series of the conformal group [50].

We will eventually restore the fishnet framework $0<\delta<\frac{d}{2}$ by analytic continuation. We also introduce, in our computations, a reference point $x_{0} \in \mathbb{R}^{d}$ (one could set it to 0 for instance). Let us define the lattice transfer-matrix $\mathbf{Q}_{N}(u)$ we want to diagonalise, where $N \in \mathbb{N}^{*}$ is the lattice width and $u \in \mathbb{C}$ is the spectral parameter. The transfer-matrix $\mathbf{Q}_{N}(u)$ is an operator acting on functions $\Phi$ of $N$ points as

$$
\begin{aligned}
& {\left[\mathbf{Q}_{N}(u) \Phi\right]\left(x_{1}, \ldots, x_{N}\right)} \\
& =\int \frac{\Phi\left(y_{1}, \ldots, y_{N}\right)}{\prod_{k=1}^{N}\left(x_{k k+1}\right)^{2 \delta}\left(y_{k}-x_{k}\right)^{2 \alpha}\left(y_{k}-x_{k-1}\right)^{2 \beta}} \prod_{j=1}^{N} \frac{\mathrm{d}^{d} y_{j}}{\pi^{\frac{d}{2}}} \\
& =\left[A_{0}(\delta) A_{0}(\alpha(u)) A_{0}(\beta(u))\right]^{N} \int \frac{\Phi\left(y_{1}, \ldots, y_{N}\right)}{\prod_{k=1}^{N}\left(w_{k}-y_{k}\right)^{2 \tilde{\delta}}\left(w_{k}-x_{k}\right)^{2 \tilde{\beta}(u)}\left(w_{k}-x_{k-1}\right)^{2 \tilde{\alpha}(u)}} \prod_{j=1}^{N} \frac{\mathrm{d}^{d} w_{j} \mathrm{~d}^{d} y_{j}}{\pi^{d}},
\end{aligned}
$$

where $x_{k k+1}=x_{k}-x_{k+1}$, and we recall that the functions $\alpha$ and $\beta$ of the spectral parameter have been defined in (2.40). The inner product between two functions of $N$ points $\Phi$ and $\Psi$ is defined by

$$
\langle\Phi \mid \Psi\rangle=\int\left\langle\Phi \mid x_{1}, \ldots, x_{N}\right\rangle\left\langle x_{1}, \ldots, x_{N} \mid \Psi\right\rangle \prod_{k=1}^{N} \frac{\mathrm{d}^{d} x_{k}}{\pi^{\frac{d}{2}}}=\int \Phi^{*}\left(x_{1}, \ldots, x_{N}\right) \Psi\left(x_{1}, \ldots, x_{N}\right) \prod_{k=1}^{N} \frac{\mathrm{d}^{d} x_{k}}{\pi^{\frac{d}{2}}} .
$$


With the definition (3.2), the constant $\pi^{-\frac{d}{2}}$ is included in the integration measure over space-time, i.e. $|x\rangle$ is such that $\langle x \mid y\rangle=\pi^{\frac{d}{2}} \delta(x-y)$. As a consequence, one can write

$$
\left\langle x_{1}, \ldots, x_{N}\left|\mathbf{Q}_{N}(u)\right| y_{1}, \ldots, y_{N}\right\rangle=\frac{1}{\prod_{k=1}^{N} x_{k, k+1}^{2 \delta}\left(y_{k}-x_{k}\right)^{2 \alpha}\left(y_{k}-x_{k-1}\right)^{2 \beta}}
$$

for the kernel of the graph-building operator, which is represented by the diagram of figure 10.

A particular case of the family of operators (3.1) - for $x_{0}=0$ - is the graph-building operator of the square-lattice fishnet

$$
\mathbf{B}_{N, \tilde{\delta}} \equiv \mathbf{Q}_{N}\left(\mathrm{i} \frac{\tilde{\delta}}{2}\right)
$$

The operators (3.1) computed at different values of the spectral parameter commute

$$
\left[\mathbf{Q}_{N}(u), \mathbf{Q}_{N}\left(u^{\prime}\right)\right]=0
$$

the proof follows all the steps of the one presented for $d=2$ dimensions in [12, 44], and it is ultimately based on the star-triangle identity (A.10). We show it in figure 11 for completeness. The notation of Feynman diagrams used in figure 10 maps lengthy manipulations of integral kernels into simple moves of lines and vertices, it will therefore be the language of many calculations of this section.

We shall construct iteratively the eigenvectors of $\mathbf{Q}_{N}$, starting from $N=1$. Since these operators commute with global rotations and dilations, the eigenvectors of $\mathbf{Q}_{1}$ are constrained to be

$$
\left\langle x \mid \mathbf{u}_{1} ; C\right\rangle=\frac{C\left(x-x_{0}\right)}{\left(x-x_{0}\right)^{2\left(\tilde{\beta}_{1}+\frac{l_{1}}{2}\right)}}, \quad \mathbf{u}_{1}=\left(u_{1}, l_{1}\right) \in \mathbb{C} \times \mathbb{N},
$$

where

$$
C(y)=C^{\mu_{1} \ldots \mu_{l_{1}}} y_{\mu_{1}} \ldots y_{\mu_{l_{1}}},
$$

and $C \in \mathbb{V}_{l}$ is a symmetric traceless tensors of rank $l_{1}$. The spectral equation reads

$$
\mathbf{Q}_{1}(u)\left|\mathbf{u}_{1} ; C\right\rangle=Q_{l_{1}}\left(u \mid u_{1}\right)\left|\mathbf{u}_{1} ; C\right\rangle
$$

and the eigenvalue, computed using the identity (A.11), is

$$
Q_{l}\left(u \mid u^{\prime}\right)=A_{0}(\alpha) A_{l}\left(\tilde{\alpha}^{\prime}\right) A_{l}\left(\beta+\tilde{\beta}^{\prime}\right)
$$

For $N>1$, we find the eigenvectors after the definition of a recursive step. For $\mathbf{u} \in \mathbb{C} \times \mathbb{N}$ and $C \in \mathbb{V}_{l}$ we introduce the layer operator $C \cdot \boldsymbol{\Lambda}_{N}(\mathbf{u})$ acting on functions of 

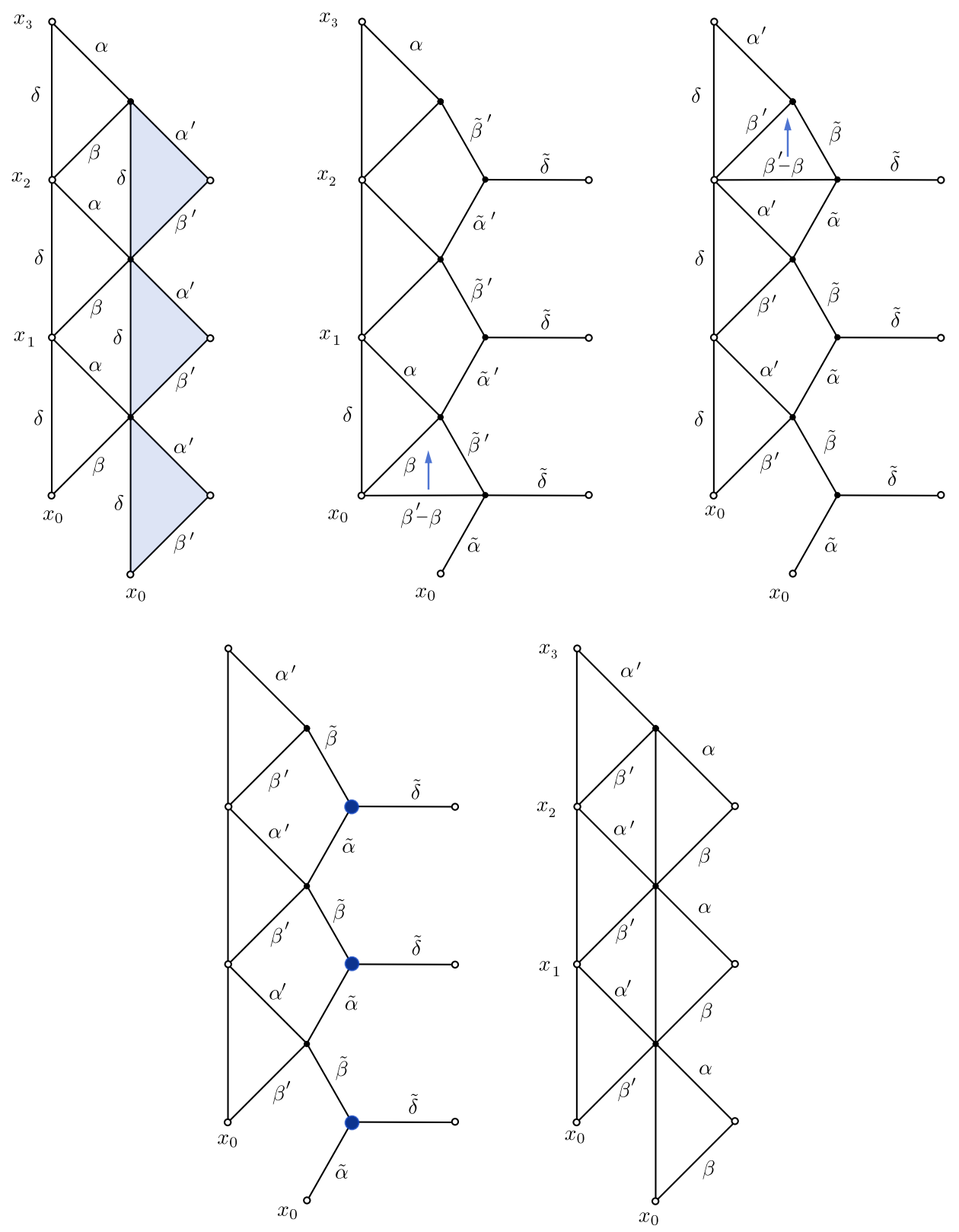

Figure 11. Commutation of operators $\mathbf{Q}_{3}$ computed at different values $u \neq u^{\prime}$ of the spectral parameter. Up left: the blue triangles are replaced by star integrals. Up center: upwards movement of the horizontal line of power $\beta-\beta^{\prime}$, by a chain of star-triangle identitites. Up right: the last passage involves star-triangle and chain-rule indentity. Down left: integration of the blue vertices by star-triangle identity. Down right: the final result is equal to the first picture with exchanged $u$ and $u^{\prime}$, i.e. $(\alpha, \beta) \leftrightarrow\left(\alpha^{\prime}, \beta^{\prime}\right)$. 
$N-1$ points and returning functions of $N$ points:

$$
\begin{aligned}
& {\left[C \cdot \boldsymbol{\Lambda}_{N}(\mathbf{u}) \Phi\right]\left(x_{1}, \ldots, x_{N}\right)} \\
& =\left[A_{0}\left(1-\alpha-\frac{l}{2}\right) A_{0}\left(\beta+\frac{l}{2}\right)\right]^{N-1} \\
& \quad \times \int \frac{C\left(\frac{x_{1}-x_{0}}{\left(x_{1}-x_{0}\right)^{2}}-\frac{y_{1}-x_{0}}{\left(y_{1}-x_{0}\right)^{2}}\right)}{\left(x_{N}-w_{N-1}\right)^{2\left(\tilde{\beta}-\frac{l}{2}\right)} \prod_{k=1}^{N-1}\left(x_{k}-w_{k}\right)^{2\left(\tilde{\alpha}+\frac{l}{2}\right)}\left(x_{k}-w_{k-1}\right)^{2\left(\tilde{\beta}-\frac{l}{2}\right)}} \\
& \quad \times \frac{\Phi\left(y_{1}, \ldots, y_{N-1}\right)}{\prod_{k=1}^{N-1}\left(y_{k}-w_{k}\right)^{2\left(\alpha+\frac{d+l}{2}-1\right)}\left(y_{k}-w_{k-1}\right)^{2\left(1-\tilde{\beta}-\frac{l}{2}\right)}} \prod_{k=1}^{N-1} \frac{\mathrm{d}^{d} w_{k}}{\pi^{\frac{d}{2}}} \frac{\mathrm{d}^{d} y_{k}}{\pi^{\frac{d}{2}}}
\end{aligned}
$$

with $w_{0}=x_{0}$. The scalar prefactor in (3.10) leads to a convenient normalisation for the eigenvectors, that simplifies the form of their symmetry property and inner products. Strictly speaking, the integrals (3.10) are ill-defined if $l>0$ and they should be understood as analytic continuations. Despite that, we can perform on them all the needed manipulations via integral identities presented in appendix A. The operator $\boldsymbol{\Lambda}_{N}(\mathbf{u})$ carries $l$ symmetric traceless tensor indices,

$$
\boldsymbol{\Lambda}_{N}(\mathbf{u})^{\mu_{1} \ldots \mu_{l}},
$$

and its pairing with the tensor $C$ can be encoded in the action of a differential operator, according to (A.4):

$$
C \cdot \boldsymbol{\Lambda}_{N}(\mathbf{u})=\frac{C\left(\nabla_{x_{0}}\right)}{2^{l}\left(\tilde{\beta}-\frac{l}{2}\right)_{l}} \Lambda_{N}(\mathbf{u}) .
$$

The kernel of this last operator $\Lambda_{N}(\mathbf{u})$ is represented by the diagram of figure 12 for $N=4$. The crucial relation satisfied by the layer operator is (see the proof in figure 13 and figure 14)

$$
\mathbf{Q}_{N}(u) C_{N} \cdot \boldsymbol{\Lambda}_{N}\left(\mathbf{u}_{N}\right)=Q_{l_{N}}\left(u \mid u_{N}\right) C_{N} \cdot \boldsymbol{\Lambda}_{N}\left(\mathbf{u}_{N}\right) \mathbf{Q}_{N-1}(u),
$$

and the eigenvectors of $\mathbf{Q}_{N}(u)$ are therefore constructed iteratively as

$$
\left|\mathbf{u}_{1}, \ldots, \mathbf{u}_{N} ; C_{1} \otimes \cdots \otimes C_{N}\right\rangle=C_{N} \cdot \boldsymbol{\Lambda}_{N}\left(\mathbf{u}_{N}\right) \cdots C_{2} \cdot \boldsymbol{\Lambda}_{2}\left(\mathbf{u}_{2}\right)\left|\mathbf{u}_{1} ; C_{1}\right\rangle
$$

with arbitrarily chosen $C_{i} \in \mathbb{V}_{l_{i}}$. The spectral equation for the graph-building transfermatrix reads

$$
\mathbf{Q}_{N}(u)\left|\mathbf{u}_{1}, \ldots, \mathbf{u}_{N} ; C\right\rangle=\prod_{k=1}^{N} Q_{l_{k}}\left(u \mid u_{k}\right)\left|\mathbf{u}_{1}, \ldots, \mathbf{u}_{N} ; C\right\rangle
$$

for an arbitrary tensor $C \in \mathbb{V}_{l_{1}} \otimes \cdots \otimes \mathbb{V}_{l_{N}}$, in agreement with the invariance of $\mathbf{Q}_{N}(u)$ under $O(d)$ rotations. The spectrum of the transfer-matrix is factorized into $N$ identical contributions of the type found at $N=1$, each depending on a rapidity $u_{i}$ and a Lorentz spin $l_{i}$, and is symmetric with respect to permutations of these quantum numbers. 

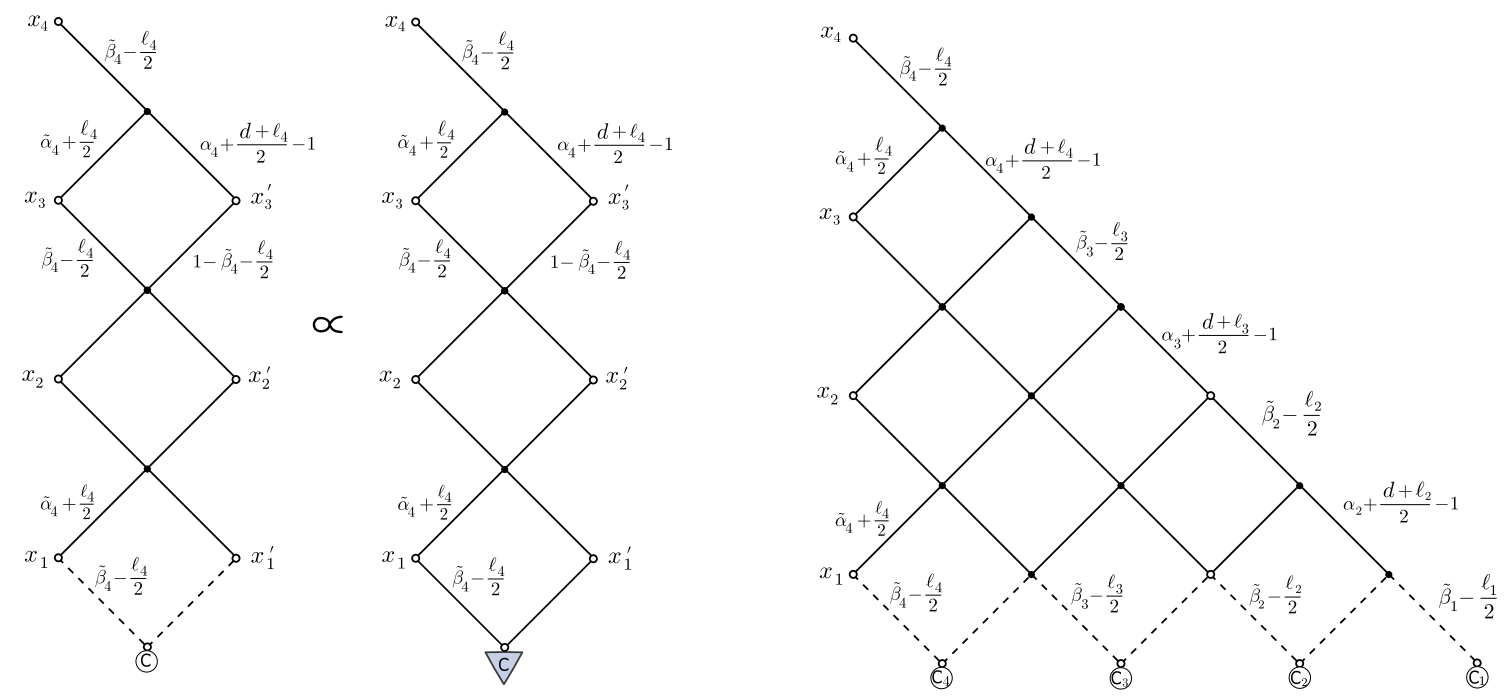

Figure 12. Left: graphical representation of the layer operator $C \cdot \boldsymbol{\Lambda}_{4}\left(\mathbf{u}_{4}\right) \propto C\left(\nabla_{x_{0}}\right) \cdot \Lambda_{4}\left(\mathbf{u}_{4}\right)$. The blue triangle stands for the differential operator $C\left(\nabla_{x_{0}}\right)$. Right: graphical representation of the eigenvector $\left|\mathbf{u}_{1}, \ldots, \mathbf{u}_{4} ; C\right\rangle$.

\section{$3.2 \quad$ Symmetry property}

The symmetry of the spectrum of $\mathbf{Q}_{N}(u)$ with respect to permutations of quantum numbers $\mathbf{u}_{i}=\left(u_{i}, l_{i}\right)$ has its counterpart at the level of the eigenvectors. Using the integral representation (2.43) of the R-matrix, it is possible to show the following commutation relation:

$$
C \cdot \boldsymbol{\Lambda}_{N+1}\left(\mathbf{u}_{1}\right) \otimes \boldsymbol{\Lambda}_{N}\left(\mathbf{u}_{2}\right)=\left[\mathbb{S}_{l_{1}, l_{2}}\left(u_{1}-u_{2}\right) C\right] \cdot \boldsymbol{\Lambda}_{N+1}\left(\mathbf{u}_{2}\right) \otimes \boldsymbol{\Lambda}_{N}\left(\mathbf{u}_{1}\right)
$$

where $N \geqslant 2, C \in \mathbb{V}_{l_{1}} \otimes \mathbb{V}_{l_{2}}$, and the tensor product notation concerns only the finitedimensional spaces $\mathbb{V}_{l_{1}}$ and $\mathbb{V}_{l_{2}}$. The matrix $\mathbb{S}_{l_{1}, l_{2}}(u)$ coincides with the fused R-matrix up to a scalar phase:

$$
\mathbb{S}_{l, l^{\prime}}(u)=(-1)^{l+l^{\prime}} \frac{\Gamma\left(1+\frac{l+l^{\prime}}{2}-\mathrm{i} u\right)}{\Gamma\left(1+\frac{l+l^{\prime}}{2}+\mathrm{i} u\right)} \frac{\Gamma\left(\frac{d}{2}-1+\frac{l+l^{\prime}}{2}-\mathrm{i} u\right)}{\Gamma\left(\frac{d}{2}-1+\frac{l+l^{\prime}}{2}+\mathrm{i} u\right)} \mathbb{R}_{l, l^{\prime}}(u) .
$$

The proof of (3.16) is heavily based on the main interchange relation (figure 4) and presented in figure 15. A very similar computation applies to the $N=1$ case, and proves that

$$
\left|\mathbf{u}_{1}, \mathbf{u}_{2} ; C\right\rangle=\left|\mathbf{u}_{2}, \mathbf{u}_{1} ; \mathbb{P}_{12} \mathbb{S}_{l_{1}, l_{2}}\left(u_{2}-u_{1}\right) C\right\rangle
$$

where $\mathbb{P}_{12}: \mathbb{V}_{l_{1}} \otimes \mathbb{V}_{l_{2}} \rightarrow \mathbb{V}_{l_{2}} \otimes \mathbb{V}_{l_{1}}$ is the exchange of the two factors.

The property (3.16) relates two eigenvectors with exchanged $\mathbf{u}_{k}, \mathbf{u}_{k+1}$. For a generic permutation $\sigma \in \mathfrak{S}_{N}$, the matrix $\mathbb{S}_{l_{k}, l_{k+1}}\left(u_{k}-u_{k+1}\right)$ is replaced by an operator $\mathbb{S}\left(\mathbf{u}_{1}, \ldots, \mathbf{u}_{N} ; \sigma\right)$ acting on $\mathbb{V}_{l_{1}} \otimes \cdots \otimes \mathbb{V}_{l_{N}}$. First, we set

$$
\mathbb{S}\left(\mathbf{u}_{1}, \ldots, \mathbf{u}_{N} ; \mathrm{id}\right)=\operatorname{Id}_{l_{1}} \otimes \cdots \otimes \operatorname{Id}_{l_{N}}
$$

moreover, if $\sigma$ is the transposition $(k, k+1)$, we impose

$$
\mathbb{S}\left(\mathbf{u}_{1}, \ldots, \mathbf{u}_{N} ;(k k+1)\right)=\operatorname{Id}_{l_{1}} \otimes \cdots \otimes \operatorname{Id}_{l_{k-1}} \otimes \mathbb{S}_{l_{k}, l_{k+1}}\left(u_{k+1}-u_{k}\right) \otimes \operatorname{Id}_{l_{k+2}} \otimes \cdots \otimes \operatorname{Id}_{l_{N}} .
$$



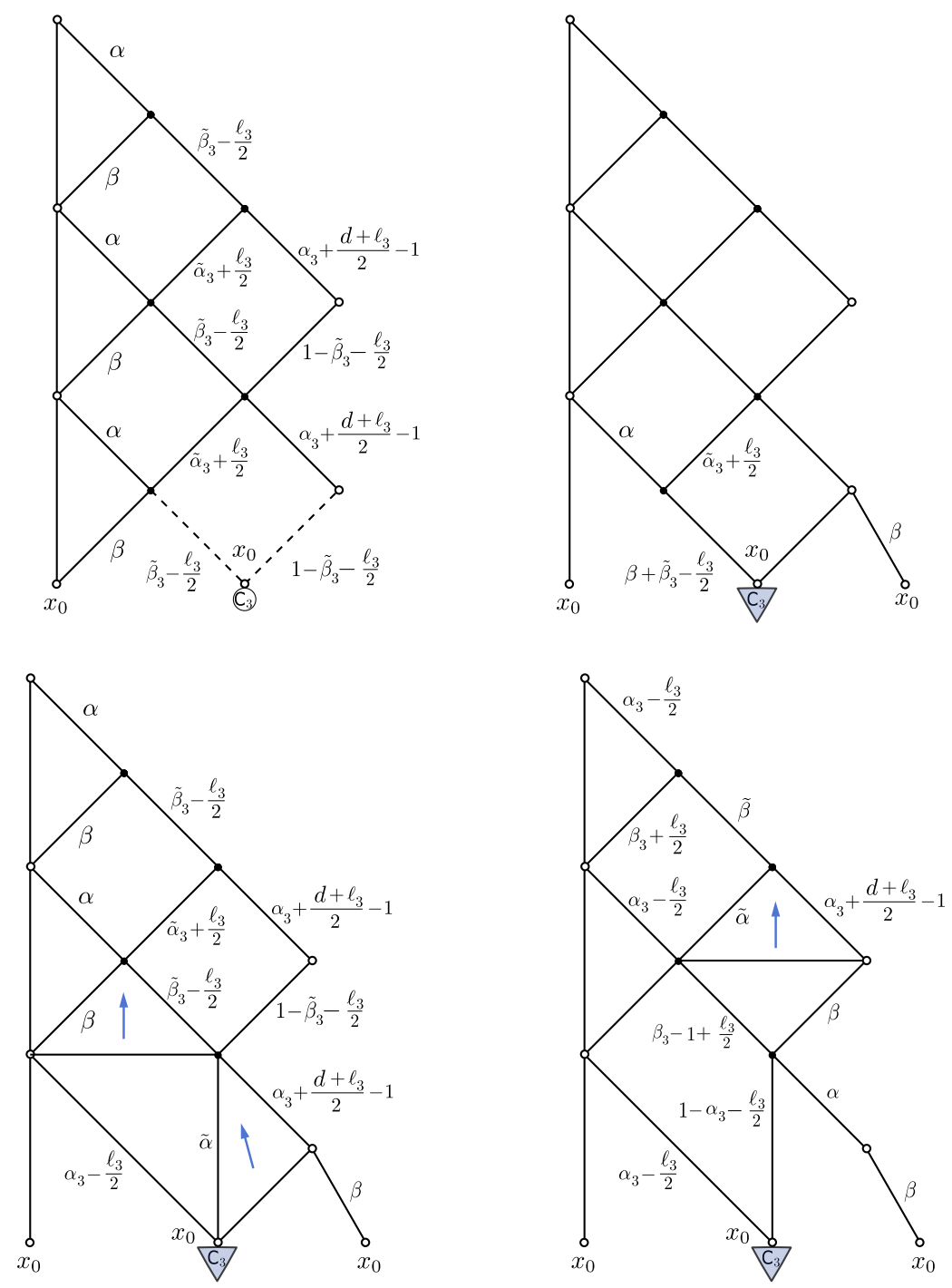

Figure 13. Top left: action of the transfer matrix $\mathbf{Q}_{3}(u)$ on the layer operator $C_{3} \cdot \boldsymbol{\Lambda}_{3}\left(u_{3}\right)$. Top right: the tensor structure is regarded as the action of a differential operator $C_{3}\left(\nabla_{x_{0}}\right)$ as in equation (A.4), and here represented by the blue triangle. The line with power $\beta$ and extreme $x_{0}$ is pulled under the action of the operator $C_{3}\left(\nabla_{x_{0}}\right)$ by means of the property (A.5). In the bottom drawings, lines are moved via several star-triangle and chain-rule identities, illustrated via arrows (see, for details, appendix A).

Finally, we require the factorisation property

$$
\mathbb{S}\left(\mathbf{u}_{1}, \ldots, \mathbf{u}_{N} ;(k k+1) \sigma\right)=\mathbb{S}\left(\mathbf{u}_{\sigma^{-1}(1)}, \ldots, \mathbf{u}_{\sigma^{-1}(N)} ;(k k+1)\right) \mathbb{S}\left(\mathbf{u}_{1}, \ldots, \mathbf{u}_{N} ; \sigma\right)
$$

for any $k \in\{1, \ldots, N-1\}$ and any permutation $\sigma$. Since any permutation can be decomposed into a product of transpositions of the form $(k k+1)$, this is enough to define $\mathbb{S}\left(\mathbf{u}_{1}, \ldots, \mathbf{u}_{\mathbf{N}} ; \sigma\right)$ for all $\sigma \in \mathfrak{S}_{N}$. Furthermore, there is no ambiguity in this definition because $\mathbb{S}_{l, l^{\prime}}$ satisfies the Yang-Baxter equation. 

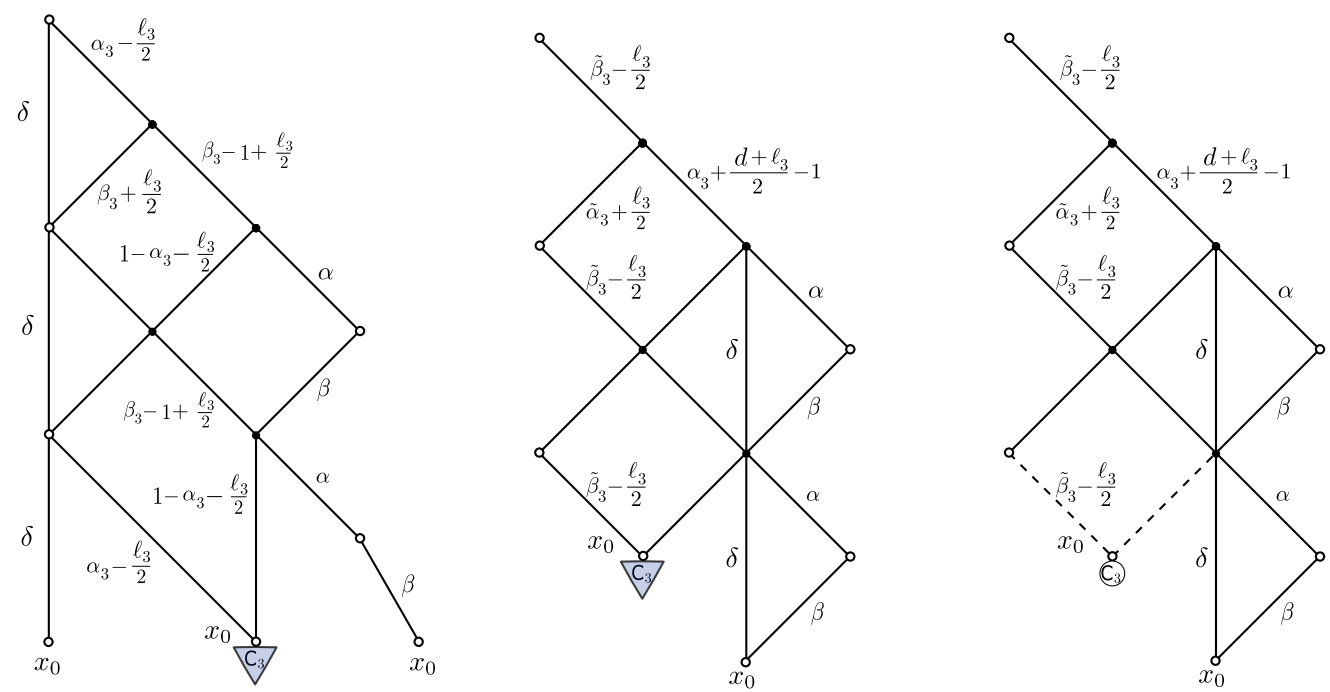

Figure 14. Last step of the diagonalisation procedure: in order to pass from the first to the second drawing, the vertical lines with indices $\delta$ have been moved to the right. The upper two were moved using star-triangle and chain relations, whereas the lower one is moved using property (A.5). The last picture corresponds to the expression $C_{3} \cdot \boldsymbol{\Lambda}_{3}\left(u_{3}\right) \mathbf{Q}_{2}(u)$.

The consequence of (3.14), (3.16), (3.18), and (3.21) on the eigenvectors is the following symmetry property: for any permutation of quantum numbers $\sigma \in \mathfrak{S}_{N}$, one has

$$
\left|\mathbf{u}_{1}, \ldots, \mathbf{u}_{N} ; C\right\rangle=\left|\mathbf{u}_{\sigma^{-1}(1)}, \ldots, \mathbf{u}_{\sigma^{-1}(N)} ; \mathbb{P}_{\sigma} \mathbb{S}\left(\mathbf{u}_{1}, \ldots, \mathbf{u}_{N} ; \sigma\right) C\right\rangle
$$

where $\mathbb{P}_{\sigma}: \mathbb{V}_{l_{1}} \otimes \cdots \otimes \mathbb{V}_{l_{N}} \rightarrow \mathbb{V}_{l_{\sigma^{-1}(1)}} \otimes \cdots \otimes \mathbb{V}_{l_{\sigma^{-1}(N)}}$ is the canonical isomorphism.

We point out that the exchange property (3.16) is one of the defining properties of the Zamolodchikovs-Faddeev algebra [20, 41]. Moreover, the symmetry property (3.22) would also be typical for eigenvectors of compact spin chains for instance. However, since we are now considering a model with continuous spectrum, the tensor $C$ and the rapidities can be chosen arbitrarily; there are no (nested) Bethe ansatz equations.

\subsection{Inner product}

The inner product for eigenvectors of the model of length $N=1$ is trivially computed to be

$$
\left\langle\mathbf{u} ; C \mid \mathbf{u}^{\prime} ; C^{\prime}\right\rangle=\int \frac{C^{*}\left(x-x_{0}\right) C^{\prime}\left(x-x_{0}\right)}{\left(x-x_{0}\right)^{2\left(\tilde{\beta}^{*}+\tilde{\beta}^{\prime}+\frac{l+l^{\prime}}{2}\right)}} \frac{\mathrm{d}^{d} x}{\pi^{\frac{d}{2}}}=\delta\left(\mathbf{u}-\mathbf{u}^{\prime}\right)\left\langle C \mid C^{\prime}\right\rangle,
$$

where $\delta\left(\mathbf{u}-\mathbf{u}^{\prime}\right)=\delta_{l l^{\prime}} \delta\left(u-u^{\prime}\right)$ and

$$
\left\langle C \mid C^{\prime}\right\rangle=\pi \int_{\mathbb{S}^{d-1}} C^{*}(n) C^{\prime}(n) \frac{\mathrm{d} n}{\pi^{\frac{d}{2}}}=\frac{l ! 2^{1-l} \pi}{\Gamma\left(\frac{d}{2}+l\right)} C_{\mu_{1} \ldots \mu_{l}}^{*} C^{\prime \mu_{1} \ldots \mu_{l}}
$$

is the inner product we choose on $\mathbb{V}_{l}$. The inner product of eigenvectors of length $N>1$ is computed based on the iterative construction via layer operators (3.10). In fact, under the assumption $\mathbf{u}^{\prime} \neq \mathbf{u}$, the overlap of two layer operators of length $N$ is expressed using 

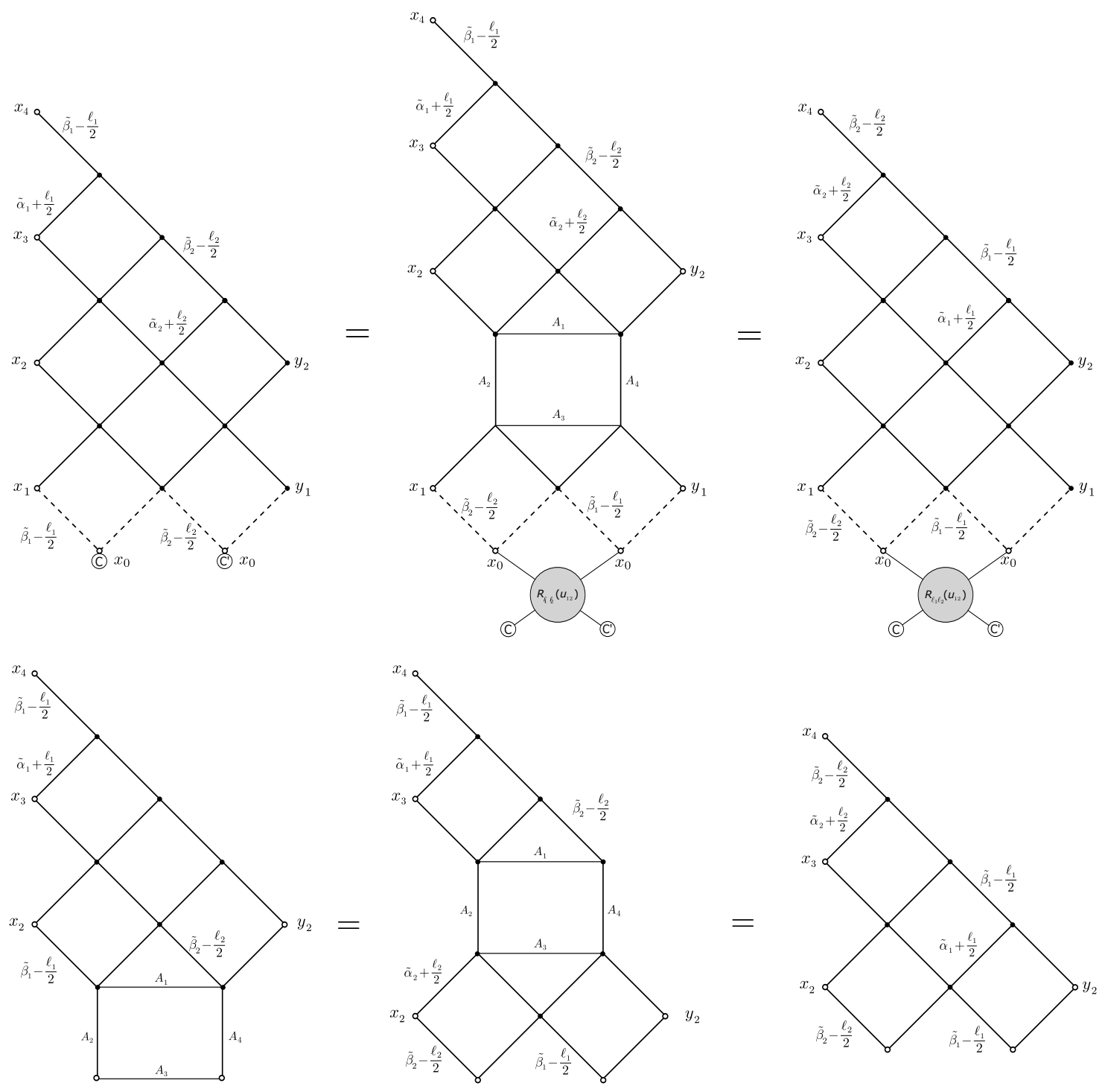

Figure 15. Proof of the symmetry of eigenvectors.

layers of length $N-1$ via

$$
\begin{aligned}
C^{\prime} \cdot \boldsymbol{\Lambda}_{N}^{\dagger}\left(\mathbf{u}^{\prime}\right) C \cdot \boldsymbol{\Lambda}_{N}(\mathbf{u}) & =\left(C^{\prime} \otimes C\right) \cdot \boldsymbol{\Lambda}_{N}^{\dagger}\left(\mathbf{u}^{\prime}\right) \otimes \boldsymbol{\Lambda}_{N}(\mathbf{u}) \\
& =\frac{\left[t_{2} \mathbb{S}_{l, l^{\prime}}\left(u-u^{\prime}\right) C \otimes C^{\prime}\right] \cdot \boldsymbol{\Lambda}_{N-1}(\mathbf{u}) \otimes \boldsymbol{\Lambda}_{N-1}^{\dagger}\left(\mathbf{u}^{\prime}\right)}{\left[\left(u-u^{\prime}\right)^{2}+\frac{\left(l-l^{\prime}\right)^{2}}{4}\right]\left[\left(u-u^{\prime}\right)^{2}+\frac{\left(d-2+l+l^{\prime}\right)^{2}}{4}\right]}, \quad \text { if } \quad N>2,
\end{aligned}
$$

and

$$
\begin{aligned}
\left\langle x\left|C^{\prime} \cdot \boldsymbol{\Lambda}_{2}^{\dagger}\left(\mathbf{u}^{\prime}\right) C \cdot \boldsymbol{\Lambda}_{2}(\mathbf{u})\right| y\right\rangle= & \frac{1}{\left[\left(u-u^{\prime}\right)^{2}+\frac{\left(l-l^{\prime}\right)^{2}}{4}\right]\left[\left(u-u^{\prime}\right)^{2}+\frac{\left(d-2+l+l^{\prime}\right)^{2}}{4}\right]} \times \\
& \times \frac{\left[{ }^{t_{2}} \mathbb{S}_{l, l^{\prime}}\left(u-u^{\prime}\right) C \otimes C^{\prime}\right] \cdot\left(x-x_{0}\right)^{\otimes l} \otimes\left(y-x_{0}\right)^{\otimes l^{\prime}}}{\left(x-x_{0}\right)^{2\left(\tilde{\beta}+\frac{l}{2}\right)}\left(y-x_{0}\right)^{2\left(\beta^{\prime}+\frac{l^{\prime}}{2}\right)}} .
\end{aligned}
$$


The properties (3.26) and (3.25) are obtained using the integral representation (2.43) of the R-matrix and crossing symmetry (2.62), and are shown by the diagrams in figure 16. From the iteration of (3.25) and the symmetry property (3.22), the overlap of two eigenvectors reads

$$
\left\langle\mathbf{u}_{1}, \ldots, \mathbf{u}_{N} ; C \mid \mathbf{u}_{1}^{\prime}, \ldots, \mathbf{u}_{N}^{\prime} ; C^{\prime}\right\rangle=\frac{\sum_{\sigma \in \mathfrak{S}_{N}} \prod_{k=1}^{N} \delta\left(\mathbf{u}_{\sigma(k)}-\mathbf{u}_{k}^{\prime}\right)\left\langle C \mid \mathbb{P}_{\sigma} \mathbb{S}\left(\mathbf{u}_{1}^{\prime}, \ldots, \mathbf{u}_{N}^{\prime} ; \sigma\right) C^{\prime}\right\rangle}{\mu\left(\mathbf{u}_{1}, \ldots, \mathbf{u}_{N}\right)},
$$

where the measure $\mu$ is defined as

$$
\mu\left(\mathbf{u}_{1}, \ldots, \mathbf{u}_{N}\right)=\prod_{1 \leqslant j<k \leqslant N}\left[\left(u_{j}-u_{k}\right)^{2}+\frac{\left(l_{j}-l_{k}\right)^{2}}{4}\right]\left[\left(u_{j}-u_{k}\right)^{2}+\frac{\left(d-2+l_{j}+l_{k}\right)^{2}}{4}\right] .
$$

Let us understand this formula with an explicit example. For $N=3$, the inner product is

$$
\begin{aligned}
& \left\langle\mathbf{u}_{1}, \mathbf{u}_{2}, \mathbf{u}_{3} ; C_{1} \otimes C_{2} \otimes C_{3} \mid \mathbf{u}_{1}^{\prime}, \mathbf{u}_{2}^{\prime}, \mathbf{u}_{3}^{\prime} ; C_{1}^{\prime} \otimes C_{2}^{\prime} \otimes C_{3}^{\prime}\right\rangle= \\
& =\left\langle\mathbf{u}_{1} ; C_{1}\left|C_{2}^{*} \cdot \boldsymbol{\Lambda}_{2}^{\dagger}\left(\mathbf{u}_{2}\right) C_{3}^{*} \cdot \boldsymbol{\Lambda}_{3}^{\dagger}\left(\mathbf{u}_{3}\right) C_{3}^{\prime} \cdot \boldsymbol{\Lambda}_{3}\left(\mathbf{u}_{3}^{\prime}\right) C_{2}^{\prime} \cdot \boldsymbol{\Lambda}_{2}\left(\mathbf{u}_{2}^{\prime}\right)\right| \mathbf{u}_{1}^{\prime} ; C_{1}^{\prime}\right\rangle= \\
& =\int\left\langle\mathbf{u}_{1} ; C_{1} \mid x\right\rangle\left\langle x\left|C_{2}^{*} \cdot \boldsymbol{\Lambda}_{2}^{\dagger}\left(\mathbf{u}_{2}\right) C_{3}^{*} \cdot \boldsymbol{\Lambda}_{3}^{\dagger}\left(\mathbf{u}_{3}\right) C_{3}^{\prime} \cdot \boldsymbol{\Lambda}_{3}\left(\mathbf{u}_{3}^{\prime}\right) C_{2}^{\prime} \cdot \boldsymbol{\Lambda}_{2}\left(\mathbf{u}_{2}^{\prime}\right)\right| y\right\rangle\left\langle y \mid \mathbf{u}_{1}^{\prime} ; C_{1}^{\prime}\right\rangle \frac{\mathrm{d}^{d} x \mathrm{~d}^{d} y}{\pi^{d}} .
\end{aligned}
$$

If we assume that $\mathbf{u}_{3} \neq \mathbf{u}_{3}^{\prime}, \mathbf{u}_{3} \neq \mathbf{u}_{2}^{\prime}$, and $\mathbf{u}_{2} \neq \mathbf{u}_{3}^{\prime}$, then, thanks the overlap formula (3.25), one can write

$$
\begin{aligned}
& \left\langle\mathbf{u}_{1}, \mathbf{u}_{2}, \mathbf{u}_{3} ; C_{1} \otimes C_{2} \otimes C_{3} \mid \mathbf{u}_{1}^{\prime}, \mathbf{u}_{2}^{\prime}, \mathbf{u}_{3}^{\prime} ; C_{1}^{\prime} \otimes C_{2}^{\prime} \otimes C_{3}^{\prime}\right\rangle \\
& \propto \int\left[t_{2} \mathbb{S}_{l_{3}^{\prime}, l_{2}}\left(u_{3}^{\prime}-u_{2}\right)^{t_{3}} \mathbb{S}_{l_{2}^{\prime}, l_{3}}\left(u_{2}^{\prime}-u_{3}\right)^{t_{3}} \mathbb{S}_{l_{3}^{\prime}, l_{3}}\left(u_{3}^{\prime}-u_{3}\right) C_{2}^{\prime} \otimes C_{3}^{\prime} \otimes C_{2}^{*} \otimes C_{3}^{*}\right](z ; x ; z ; y) \\
& \quad \times \frac{C_{1}^{*}(x) C_{1}^{\prime}(y)}{x^{2\left(\tilde{\beta}_{1}^{*}+\tilde{\beta}_{3}^{\prime}+\frac{l_{1}+l_{3}^{\prime}}{2}\right)} y_{y}{ }^{2\left(\tilde{\beta}_{3}^{*}+\tilde{\beta}_{1}^{\prime}+\frac{l_{3}+l_{1}^{\prime}}{2}\right)} z_{z}^{2\left(\tilde{\beta}_{2}^{*}+\tilde{\beta}_{2}^{\prime}+\frac{l_{2}+l_{2}^{\prime}}{2}\right)} \frac{\mathrm{d}^{d} x \mathrm{~d}^{d} y \mathrm{~d}^{d} z}{\pi^{\frac{3 d}{2}}}} .
\end{aligned}
$$

We have used the following notation: for $C \in \mathbb{V}_{l_{1}} \otimes \cdots \otimes \mathbb{V}_{l_{N}}$ and $x_{1}, \ldots, x_{N}$ arbitrary

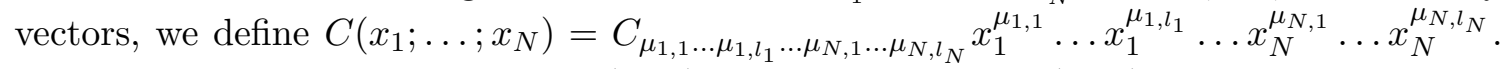
The integrals over $x, y$, and $z$ in (3.30) are of the form of (3.23), and their computation yields

$$
\begin{aligned}
& \left\langle\mathbf{u}_{1}, \mathbf{u}_{2}, \mathbf{u}_{3} ; C_{1} \otimes C_{2} \otimes C_{3} \mid \mathbf{u}_{1}^{\prime}, \mathbf{u}_{2}^{\prime}, \mathbf{u}_{3}^{\prime} ; C_{1}^{\prime} \otimes C_{2}^{\prime} \otimes C_{3}^{\prime}\right\rangle \propto \delta\left(\mathbf{u}_{1}-\mathbf{u}_{3}^{\prime}\right) \delta\left(\mathbf{u}_{2}-\mathbf{u}_{2}^{\prime}\right) \delta\left(\mathbf{u}_{3}-\mathbf{u}_{1}^{\prime}\right) \\
& \times\left\langle C_{3} \otimes C_{2} \otimes C_{1} \mid \mathbb{S}_{l_{2}^{\prime}, l_{3}^{\prime}}\left(u_{3}^{\prime}-u_{2}^{\prime}\right) \mathbb{S}_{l_{1}^{\prime}, l_{3}^{\prime}}\left(u_{3}^{\prime}-u_{1}^{\prime}\right) \mathbb{S}_{l_{1}^{\prime}, l_{2}^{\prime}}\left(u_{2}^{\prime}-u_{1}^{\prime}\right) C_{1}^{\prime} \otimes C_{2}^{\prime} \otimes C_{3}^{\prime}\right\rangle
\end{aligned}
$$

Thanks to the delta functions, the prefactor is actually exactly $\mu\left(\mathbf{u}_{1}, \mathbf{u}_{2}, \mathbf{u}_{3}\right)^{-1}$. It remains to notice that

$\mathbb{S}_{l_{2}^{\prime}, l_{3}^{\prime}}\left(u_{3}^{\prime}-u_{2}^{\prime}\right) \mathbb{S}_{l_{1}^{\prime}, l_{3}^{\prime}}\left(u_{3}^{\prime}-u_{1}^{\prime}\right) \mathbb{S}_{l_{1}^{\prime}, l_{2}^{\prime}}\left(u_{2}^{\prime}-u_{1}^{\prime}\right)=\mathbb{S}\left(\mathbf{u}_{1}^{\prime}, \mathbf{u}_{2}^{\prime}, \mathbf{u}_{3}^{\prime} ;(12)(23)(12)\right)=\mathbb{S}\left(\mathbf{u}_{1}^{\prime}, \mathbf{u}_{2}^{\prime}, \mathbf{u}_{3}^{\prime} ;\right.$ 
because of (3.21). On the other hand, when $\mathbf{u}_{3} \neq \mathbf{u}_{3}^{\prime}, \mathbf{u}_{3} \neq \mathbf{u}_{2}^{\prime}$, and $\mathbf{u}_{2} \neq \mathbf{u}_{3}^{\prime}$, formula (3.27) also reduces to

$$
\begin{aligned}
& \left\langle\mathbf{u}_{1}, \mathbf{u}_{2}, \mathbf{u}_{3} ; C_{1} \otimes C_{2} \otimes C_{3} \mid \mathbf{u}_{1}^{\prime}, \mathbf{u}_{2}^{\prime}, \mathbf{u}_{3}^{\prime} ; C_{1}^{\prime} \otimes C_{2}^{\prime} \otimes C_{3}^{\prime}\right\rangle=\mu\left(\mathbf{u}_{1}, \mathbf{u}_{2}, \mathbf{u}_{3}\right)^{-1} \\
& \times \delta\left(\mathbf{u}_{1}-\mathbf{u}_{3}^{\prime}\right) \delta\left(\mathbf{u}_{2}-\mathbf{u}_{2}^{\prime}\right) \delta\left(\mathbf{u}_{3}-\mathbf{u}_{1}^{\prime}\right)\left\langle C_{3} \otimes C_{2} \otimes C_{1} \mid \mathbb{S}\left(\mathbf{u}_{1}^{\prime}, \mathbf{u}_{2}^{\prime}, \mathbf{u}_{3}^{\prime} ;(13)\right) C_{1}^{\prime} \otimes C_{2}^{\prime} \otimes C_{3}^{\prime}\right\rangle .
\end{aligned}
$$

The other terms of (3.27) appear when requiring, following (3.22), that the full result for the inner product be invariant under

$$
\left|\mathbf{u}_{1}, \mathbf{u}_{2}, \mathbf{u}_{3} ; C_{1} \otimes C_{2} \otimes C_{3}\right\rangle \longmapsto\left|\mathbf{u}_{\sigma^{-1}(1)}, \mathbf{u}_{\sigma^{-1}(2)}, \mathbf{u}_{\sigma^{-1}(3)} ; \mathbb{P}_{\sigma} \mathbb{S}\left(\mathbf{u}_{1}, \mathbf{u}_{2}, \mathbf{u}_{3} ; \sigma\right) C_{1} \otimes C_{2} \otimes C_{3}\right\rangle
$$

for all the permutations $\sigma \in \mathfrak{S}_{3}$. This whole procedure is generalized to arbitrary $N$ thanks the iterative form of the property (3.25).

\subsection{Completeness}

Let us fix $\left\{C_{m, l}\right\}_{1 \leqslant m \leqslant d_{l}}$ an orthonormal basis of $\mathbb{V}_{l}$ with respect to the inner product defined in (3.24) $\left(d_{l}\right.$ is the dimension of $\left.\mathbb{V}_{l}\right)$. We postulate that for any $N$, the following resolution of the identity holds:

$$
\begin{aligned}
& \sum_{\substack{0 \leqslant l_{1}<+\infty \\
1 \leqslant m_{1} \leqslant d_{l_{1}}}} \ldots \sum_{\substack{0 \leqslant l_{N}<+\infty \\
1 \leqslant m_{N} \leqslant d_{l_{N}}}} \int \ldots \int \frac{\mu\left(\mathbf{u}_{1}, \ldots, \mathbf{u}_{N}\right)}{N !}\left\langle x_{1}, \ldots, x_{N} \mid \mathbf{u}_{1}, \ldots, \mathbf{u}_{N} ; C_{m_{1}, l_{1}} \otimes \cdots \otimes C_{m_{N}, l_{N}}\right\rangle \\
& \times\left\langle\mathbf{u}_{1}, \ldots, \mathbf{u}_{N} ; C_{m_{1}, l_{1}} \otimes \cdots \otimes C_{m_{N}, l_{N}} \mid y_{1}, \ldots, y_{N}\right\rangle \prod_{k=1}^{N} \mathrm{~d} u_{k}=\prod_{k=1}^{N} \pi^{\frac{d}{2}} \delta\left(x_{k}-y_{k}\right) .
\end{aligned}
$$

The integrals are over the real line: $u_{k} \in \mathbb{R}$. The power of $\pi$ in the right-hand side comes from the fact that we have defined $|x\rangle$ such that $\langle x \mid y\rangle=\pi^{\frac{d}{2}} \delta(x-y)$ (see beginning of section 3). This completeness relation is easily verified in the case $N=1$, as it coincides with the expansion of a radial function in $d$-dimensions in Gegenbauer polynomials on the sphere $S^{d-1}$. We conjecture its validity for $N>1$.

\section{Basso-Dixon diagrams}

In this section, we investigate the application of obtained basis of eigenvectors and corresponding spectral decomposition of the graph-building operator to the computations of some fishnet Feynman integrals presented in figure 17. Up to a trivial normalization factor the Feynman graph of the left panel has an interpretation as a four-point correlator in the fishnet theory:

$$
G_{M, N}^{(d, \delta)}\left(x_{1}, x_{2}, x_{3}, x_{4}\right) \propto\left\langle\operatorname{Tr}\left(X^{N}\left(x_{1}\right) Z^{M}\left(x_{2}\right) X^{\dagger N}\left(x_{3}\right) Z^{\dagger M}\left(x_{4}\right)\right)\right\rangle .
$$

Because of the conformal invariance of the integral it is equivalent to compute the integral associated to the right panel of the figure. A simple change of variables indeed shows that

$$
G_{M, N}^{(d, \delta)}\left(x_{1}, x_{2}, x_{3}, x_{4}\right)=\frac{1}{\left(x_{24}^{2}\right)^{M \delta}\left(x_{14}^{2} x_{34}^{2}\right)^{N \tilde{\delta}}} I_{M, N}^{(d, \delta)}\left(\frac{x_{14}}{x_{14}^{2}}-\frac{x_{24}}{x_{24}^{2}}, \frac{x_{34}}{x_{34}^{2}}-\frac{x_{24}}{x_{24}^{2}}\right) .
$$



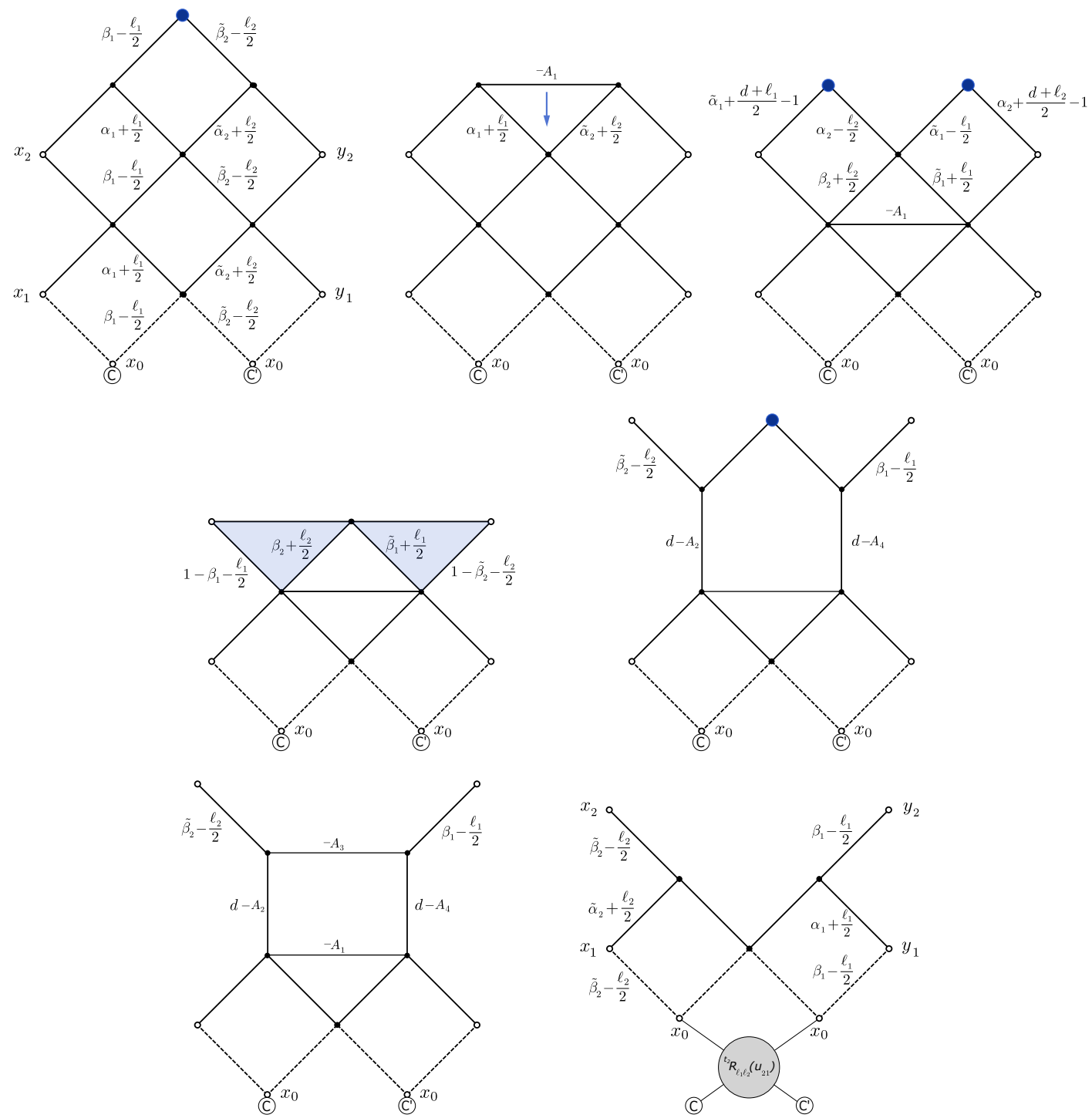

Figure 16. Proof of the inner product formula. Up left to down right: the overlap of two layer operators $C^{\prime} \cdot \boldsymbol{\Lambda}_{3}\left(u_{2}\right)$ and $C \cdot \boldsymbol{\Lambda}_{3}\left(u_{1}\right)$ is transformed via application of star-triangle identities and, at the last step, the interchange relation. The notation of blue dots, blue triangles and arrows is the same as in figure 11 and refers to different application of the star-triangle and chain-rule identities. The final expression coincides with the r.h.s. of (3.25). It shows that the overlap of two layers amounts to the scattering of the two excitations $\mathbf{u}_{\mathbf{1}}$ and $\mathbf{u}_{\mathbf{2}}$ across each other, this is expressed by the fused R-matrix $\mathbb{R}_{l_{1}, l_{2}}\left(u_{12}\right)$. 

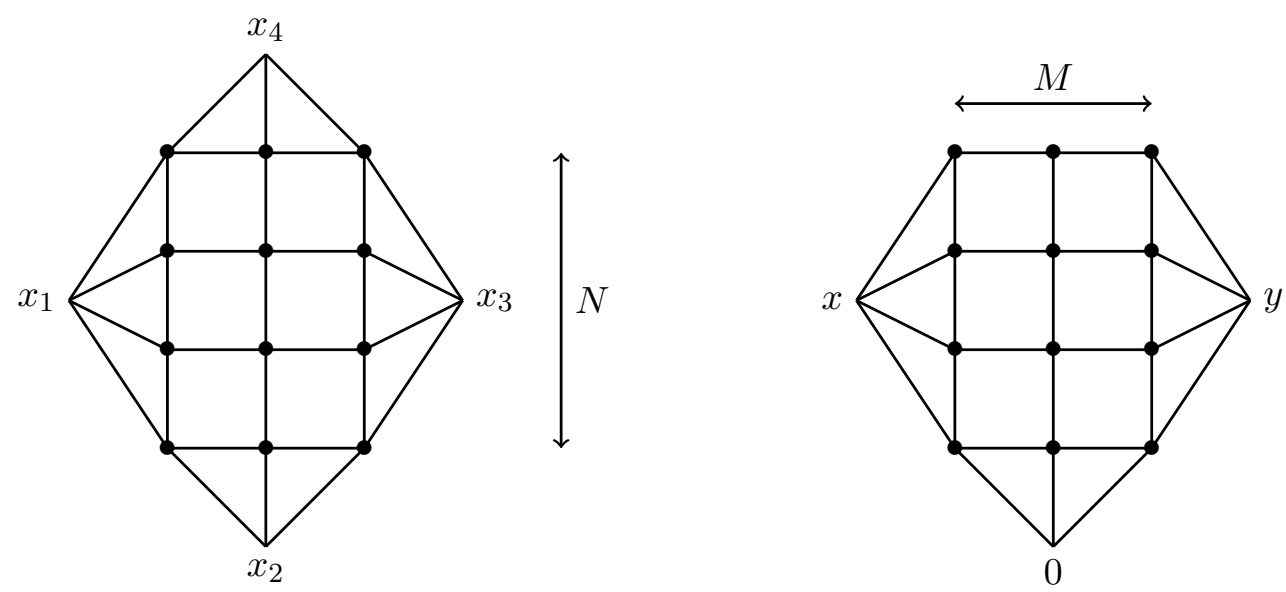

Figure 17. Feyman graphs investigated in section 4 in the case $(M, N)=(3,4)$. We call $G_{M, N}^{(d, \delta)}\left(x_{1}, x_{2}, x_{3}, x_{4}\right)$ and $I_{M, N}^{(d, \delta)}(x, y)$ the integrals represented by the graph on the left and on the right respectively. All the vertical (or ending on $x_{2}, x_{4}$ or 0 ) segments have weight $\delta$ whereas all the horizontal (or ending on $x_{1}, x_{3}, x$ or $y$ ) ones have weight $\tilde{\delta}$.

In turn, the integral $I_{M, N}^{(d, \delta)}$ is almost a matrix element of the $(M+1)$-th power of the graph-building operator $\mathbf{B}_{N, \tilde{\delta}}=\mathbf{Q}_{N}\left(\mathrm{i} \frac{\tilde{\delta}}{2}\right)$ :

$$
I_{M, N}^{(d, \delta)}(x, y)=\pi^{\frac{N d}{2}}\left\langle x, \ldots, x\left|\left(\prod_{i=1}^{N} \hat{x}_{i-1, i}^{2 \delta}\right) \mathbf{B}_{N, \tilde{\delta}}^{M+1}\right| y, \ldots, y\right\rangle
$$

where we have set $x_{0}=0$, and $\hat{x}_{i-1, i}^{2}$ is the operator of multiplication by $x_{i-1, i}^{2}$. It thus seems natural to use the spectral decomposition of the graph-building operator $\mathbf{Q}_{N}\left(\mathrm{i} \frac{\tilde{\delta}}{2}\right)$ to try to express these integrals in a simpler form. This was successfully achieved in two dimensions in [12] and in four dimensions in [14, 15]. For higher dimensions the result is actually more complicated and we are going to discuss it in a separate paper. Now we shall consider the simplest examples to illustrate how the general scheme works in the case of higher dimensions.

As we have seen above, the eigenvalue of $\mathbf{Q}_{N}\left(\mathrm{i} \frac{\tilde{\delta}}{2}\right)$ factorises into a product of

$$
Q_{l}(u)=Q_{l}\left(\mathrm{i} \frac{\tilde{\delta}}{2} \mid u\right)=\frac{\Gamma(\delta) \Gamma\left(\frac{d}{4}-\frac{\delta}{2}+\frac{l}{2}-\mathrm{i} u\right) \Gamma\left(\frac{d}{4}-\frac{\delta}{2}+\frac{l}{2}+\mathrm{i} u\right)}{\Gamma(\tilde{\delta}) \Gamma\left(\frac{d}{4}+\frac{\delta}{2}+\frac{l}{2}+\mathrm{i} u\right) \Gamma\left(\frac{d}{4}+\frac{\delta}{2}+\frac{l}{2}-\mathrm{i} u\right)} .
$$

\subsection{Ladder diagrams}

We first give the expressions for the so-called ladder diagrams [51-54] in arbitrary dimension:

$$
I_{M, 1}^{(d, \delta)}(x, y)=\frac{\Gamma\left(\frac{d}{2}\right)}{\left(x^{2} y^{2}\right)^{\frac{\tilde{\delta}}{2}}} \sum_{l=0}^{+\infty} \frac{2 l+d-2}{d-2} C_{l}^{\left(\frac{d-2}{2}\right)}(\cos \theta) \int \frac{\mathrm{d} u}{2 \pi}\left(\frac{x^{2}}{y^{2}}\right)^{\mathrm{i} u} Q_{l}^{M+1}(u),
$$


where $Q_{l}$ is given in equation (4.4), $C_{l}^{(\mu)}$ are Gegenbauer polynomials and $\cos \theta=x \cdot y /|x||y|$. We assume $d \geqslant 3$. This formula is a straightforward consequence of the completeness formula (3.35) and (4.13) below, hence we omit its proof.

The integral is straightforwardly computed by residues but the eigenvalue $Q_{l}$ generically has infinitely many poles. However, when $\delta$ is a positive integer, $Q_{l}^{-1}$ is a polynomial of degree $2 \delta$ and there is a finite number of poles. If $\delta=1$, one has $Q_{l}(u)^{-1}=$ $\Gamma\left(\frac{d-2}{2}\right)\left(u^{2}+(l+(d-2) / 2)^{2} / 4\right)$ and performing the integral yields

$$
I_{M, 1}^{(d, 1)}(x, y)=\frac{\Gamma\left(\frac{d-2}{2}\right)^{-M}}{\left(x^{2} y^{2}\right)^{\frac{d-2}{4}}} \sum_{k=M}^{2 M} \frac{k !(-2 \ln r)^{2 M-k}}{M !(k-M) !(2 M-k) !} \sum_{l=0}^{+\infty} C_{l}^{\left(\frac{d-2}{2}\right)}(\cos \theta) \frac{r^{l+\frac{d-2}{2}}}{\left(l+\frac{d-2}{2}\right)^{k}}
$$

with $r=\sqrt{y^{2} / x^{2}}$.

When $d$ is even we also have the following property of the Gegenbauer polynomials

$$
\Gamma\left(\frac{d-2}{2}\right) C_{l}^{\left(\frac{d-2}{2}\right)}(x)=2^{\frac{4-d}{2}}\left(\frac{\mathrm{d}}{\mathrm{d} x}\right)^{\frac{d-4}{2}}\left[C_{l+\frac{d-4}{2}}^{(1)}(x)\right] .
$$

Consequently, for even $d>2$, we can write $\left(z=r \mathrm{e}^{\mathrm{i} \theta}\right)$

$$
I_{M, 1}^{(d, 1)}(x, y)=\frac{2^{\frac{4-d}{2}} \Gamma\left(\frac{d-2}{2}\right)^{-M-1}}{\left(x^{2} y^{2}\right)^{\frac{d-2}{4}}}\left(\frac{\mathrm{d}}{\mathrm{d} \cos \theta}\right)^{\frac{d-4}{2}}\left[\frac{L_{M}(z, \bar{z})}{\mathrm{e}^{\mathrm{i} \theta}-\mathrm{e}^{-\mathrm{i} \theta}}\right],
$$

where we have introduced the ladder function $L_{M}$ defined for $M>0$ by

$$
L_{M}(z, \bar{z})=\sum_{k=M}^{2 M} \frac{k ![-\ln (z \bar{z})]^{2 M-k}}{M !(k-M) !(2 M-k) !}\left[\operatorname{Li}_{k}(z)-\operatorname{Li}_{k}(\bar{z})\right]
$$

with $\operatorname{Li}_{k}(z)=\sum_{n=1}^{\infty} \frac{z^{n}}{n^{k}}$ the polylogarithm.

\subsection{Two-layer diagrams}

Inserting the resolution of the identity in the expression (4.3) of $I_{M, 2}^{(d, \delta)}$, it becomes

$$
\begin{aligned}
I_{M, 2}^{(d, \delta)}(x, y)= & \sum_{\substack{0 \leqslant l_{1}<+\infty \\
1 \leqslant m_{1} \leqslant d_{l_{1}}}} \sum_{\substack{0 \leqslant l_{2}<+\infty \\
1 \leqslant m_{2} \leqslant d_{l_{2}}}} \int \mathrm{d} u_{1} \mathrm{~d} u_{2}\left[Q_{l_{1}}\left(u_{1}\right) Q_{l_{2}}\left(u_{2}\right)\right]^{M+1} \frac{\mu\left(\mathbf{u}_{1}, \mathbf{u}_{2}\right)}{2} \\
& \times\left\langle x, x\left|\hat{x}_{12}^{2 \delta} \hat{x}_{1}^{2 \delta}\right| \mathbf{u}_{1}, \mathbf{u}_{2} ; C_{m_{1}, l_{1}} \otimes C_{m_{2}, l_{2}}\right\rangle\left\langle\mathbf{u}_{1}, \mathbf{u}_{2} ; C_{m_{1}, l_{1}} \otimes C_{m_{2}, l_{2}} \mid y, y\right\rangle
\end{aligned}
$$

One can show that

$$
\begin{gathered}
\left\langle x, x\left|\hat{x}_{12}^{2 \delta} \hat{x}_{1}^{2 \delta}\right| \mathbf{u}_{1}, \mathbf{u}_{2} ; C\right\rangle=A_{0}(\tilde{\delta}) A_{l_{1}}\left(\tilde{\alpha}_{1}\right) A_{l_{2}}\left(\tilde{\alpha}_{2}\right) \frac{4^{1-\frac{d}{2}+\mathrm{i} u_{21}} \mathrm{i}^{l_{1}+l_{2}}}{x^{2\left(2 \alpha_{1}-\frac{d}{2}+1\right)}} \int \frac{\mathrm{d}^{d} p}{\pi^{\frac{d}{2}}} \frac{C(p, p) \mathrm{e}^{\mathrm{i} p \cdot x}}{p^{2\left(1+\frac{l_{1}+l_{2}}{2}+\mathrm{i} u_{21}\right)}}, \\
\left\langle y, y \mid \mathbf{u}_{1}, \mathbf{u}_{2} ; C\right\rangle=A_{0}\left(\frac{d}{2}+\delta\right) A_{l_{1}}\left(\beta_{1}\right) A_{l_{2}}\left(\beta_{2}\right) \frac{4^{1-\frac{d}{2}+\mathrm{i} u_{21}} \mathrm{i}^{l_{1}+l_{2}}}{y^{2\left(2 \tilde{\beta}_{1}-\frac{d}{2}+1\right)}} \int \frac{\mathrm{d}^{d} p}{\pi^{\frac{d}{2}}} \frac{C(p, p) \mathrm{e}^{\mathrm{i} p \cdot y}}{p^{2\left(1+\frac{l_{1}+l_{2}}{2}+\mathrm{i} u_{21}\right)}},
\end{gathered}
$$


where $u_{21}=-u_{12}=u_{2}-u_{1}$. Both equations can be rewritten using the operator $\mathbb{O}_{l_{1}, l_{2}}$ introduced in (2.56), the first one becomes for instance

$$
\left\langle x, x\left|\hat{x}_{12}^{2 \delta} \hat{x}_{1}^{2 \delta}\right| \mathbf{u}_{1}, \mathbf{u}_{2} ; C\right\rangle=A_{0}(\tilde{\delta}) A_{l_{1}}\left(\tilde{\alpha}_{1}\right) A_{l_{2}}\left(\tilde{\alpha}_{2}\right)(-1)^{l_{1}+l_{2}} A_{l_{1}+l_{2}}\left(1+\mathrm{i} u_{21}\right) \frac{\left[\mathbb{O}_{l_{1}, l_{2}}\left(u_{21}\right) C\right] \cdot x^{\otimes\left(l_{1}+l_{2}\right)}}{x^{2\left(\alpha_{1}+\alpha_{2}+\frac{l_{1}+l_{2}}{2}\right)}}
$$

If we don't want to use the operator $\mathbb{O}_{l_{1}, l_{2}}$, we have to remark that, necessarily, whatever orthonormal basis of symmetric traceless tensors we chose,

$$
\sum_{m=1}^{d_{l}} C_{m, l}(p) C_{m, l}^{*}(q)=\frac{\Gamma\left(\frac{d}{2}\right)(2 l+d-2)}{2 \pi(d-2)}|p|^{l}|q|^{l} C_{l}^{\left(\frac{d-2}{2}\right)}\left(\frac{p \cdot q}{|p||q|}\right)
$$

so that it is possible to rewrite $I_{M, 2}^{(d, \delta)}$ as

$$
\begin{aligned}
I_{M, 2}^{(d, \delta)}(x, y)= & \frac{\Gamma\left(\frac{d}{2}\right)^{2}}{2} \sum_{l_{1}, l_{2}} \int \frac{\mathrm{d} u_{1} \mathrm{~d} u_{2}}{(2 \pi)^{2}} \frac{4^{2-d}\left[Q_{l_{1}}\left(u_{1}\right) Q_{l_{2}}\left(u_{2}\right)\right]^{M+2}}{x^{2\left(2 \alpha_{1}-\frac{d}{2}+1\right)} y^{2\left(2 \beta_{1}-\frac{d}{2}+1\right)}} \\
& \times\left[u_{12}^{2}+\frac{l_{12}^{2}}{4}\right]\left[u_{12}^{2}+\frac{\left(l_{1}+l_{2}+d-2\right)^{2}}{4}\right] \frac{\left(2 l_{1}+d-2\right)\left(2 l_{2}+d-2\right)}{(d-2)^{2}} \\
& \times \int \frac{\mathrm{d}^{d} p \mathrm{~d}^{d} q}{\pi^{d}} \frac{C_{l_{1}}^{\left(\frac{d-2}{2}\right)}\left(\frac{p \cdot q}{|p| q \mid}\right) C_{l_{2}}^{\left(\frac{d-2}{2}\right)}\left(\frac{p \cdot q}{|p| q \mid}\right)}{p^{2\left(1+\mathrm{i} u_{21}\right)} q^{2\left(1+\mathrm{i} u_{12}\right)}} \mathrm{e}^{\mathrm{i} p \cdot x-\mathrm{i} q \cdot y} .
\end{aligned}
$$

In order to perform the integrals over $p$ and $q$, we may proceed as follows: first one expands the product of two Gegenbauer polynomials according to [55]

$$
C_{l_{1}}^{\left(\frac{d-2}{2}\right)}\left(\frac{p \cdot q}{|p||q|}\right) C_{l_{2}}^{\left(\frac{d-2}{2}\right)}\left(\frac{p \cdot q}{|p||q|}\right)=\sum_{m=0}^{\min \left(l_{1}, l_{2}\right)} a_{l_{1}, l_{2}, m} C_{l_{1}+l_{2}-2 m}^{\left(\frac{d-2}{2}\right)}\left(\frac{p \cdot q}{|p||q|}\right)
$$

with

$$
a_{l_{1}, l_{2}, m}=\frac{\left(l_{1}+l_{2}-2 m+\frac{d-2}{2}\right)\left(l_{1}+l_{2}-2 m\right) !}{\left(l_{1}+l_{2}-m+\frac{d-2}{2}\right) m !\left(l_{1}-m\right) !\left(l_{2}-m\right) !} \frac{\left(\frac{d-2}{2}\right)_{m}\left(\frac{d-2}{2}\right)_{l_{1}-m}\left(\frac{d-2}{2}\right)_{l_{2}-m}}{\left(\frac{d-2}{2}\right)_{l_{1}+l_{2}-m}(d-2)_{l_{1}+l_{2}-2 m}}(d-2)_{l_{1}+l_{2}-m} .
$$

Then one uses the fact that $C_{l}^{\left(\frac{d-2}{2}\right)}\left(\frac{p \cdot q}{|p||q|}\right)$ is a spherical harmonic with respect to both $p$ and $q$ (see equation (4.13)) to compute the integrals over these variables using (A.7):

$$
\int \frac{\mathrm{d}^{d} p \mathrm{~d}^{d} q}{\pi^{d}} \frac{C_{l}^{\left(\frac{d-2}{2}\right)}\left(\frac{p \cdot q}{|p||q|}\right)}{p^{2\left(1-\mathrm{i} u_{12}\right)} q^{2\left(1+\mathrm{i} u_{12}\right)}} \mathrm{e}^{\mathrm{i} p \cdot x-\mathrm{i} q \cdot y}=\left|\frac{\Gamma\left(\frac{l+d-2}{2}+\mathrm{i} u_{12}\right)}{\Gamma\left(\frac{l+2}{2}+\mathrm{i} u_{12}\right)}\right|^{2} \frac{4^{d-2} C_{l}^{\left(\frac{d-2}{2}\right)}\left(\frac{x \cdot y}{|x||y|}\right)}{x^{2\left(\frac{d}{2}-1+\mathrm{i} u_{12}\right)} y^{2\left(\frac{d}{2}-1-\mathrm{i} u_{12}\right)}} .
$$


Consequently, one can write

$$
\begin{aligned}
I_{M, 2}^{(d, \delta)}(x, y)= & \frac{\Gamma\left(\frac{d}{2}\right)^{2}}{2\left(x^{2} y^{2}\right)^{\tilde{\delta}}} \sum_{l_{1}, l_{2}} \int \frac{\mathrm{d} u_{1} \mathrm{~d} u_{2}}{(2 \pi)^{2}}\left(\frac{x^{2}}{y^{2}}\right)^{\mathrm{i}\left(u_{1}+u_{2}\right)}\left[Q_{l_{1}}\left(u_{1}\right) Q_{l_{2}}\left(u_{2}\right)\right]^{M+2} \\
& \times\left[u_{12}^{2}+\frac{l_{12}^{2}}{4}\right]\left[u_{12}^{2}+\frac{\left(l_{1}+l_{2}+d-2\right)^{2}}{4}\right] \frac{\left(2 l_{1}+d-2\right)\left(2 l_{2}+d-2\right)}{(d-2)^{2}} \\
& \times \sum_{m=0}^{\min \left(l_{1}, l_{2}\right)} a_{l_{1}, l_{2}, m}\left|\frac{\Gamma\left(\frac{l_{1}+l_{2}-2 m+d-2}{2}+\mathrm{i} u_{12}\right)}{\Gamma\left(\frac{l_{1}+l_{2}-2 m+2}{2}+\mathrm{i} u_{12}\right)}\right|^{2} C_{l_{1}+l_{2}-2 m}^{\left(\frac{d-2}{2}\right)}(\cos \theta)
\end{aligned}
$$

with $x \cdot y=|x||y| \cos \theta$. Using the operator $\mathbb{O}_{l_{1}, l_{2}}$, this can actually be written in a more concise way:

$$
\begin{aligned}
I_{M, 2}^{(d, \delta)}(x, y)= & \frac{\Gamma\left(\frac{d}{2}\right)^{2}}{2\left(x^{2} y^{2}\right)^{\tilde{\delta}}} \sum_{l_{1}, l_{2}} \int \frac{\mathrm{d} u_{1} \mathrm{~d} u_{2}}{(2 \pi)^{2}}\left(\frac{x^{2}}{y^{2}}\right)^{\mathrm{i}\left(u_{1}+u_{2}\right)}\left[Q_{l_{1}}\left(u_{1}\right) Q_{l_{2}}\left(u_{2}\right)\right]^{M+2} \\
& \times\left[u_{12}^{2}+\frac{l_{12}^{2}}{4}\right]\left[u_{12}^{2}+\frac{\left(l_{1}+l_{2}+d-2\right)^{2}}{4}\right] \frac{\left(2 l_{1}+d-2\right)\left(2 l_{2}+d-2\right)}{(d-2)^{2}} \\
& \times\left|\frac{\Gamma\left(\frac{l_{1}+l_{2}+d-2}{2}+\mathrm{i} u_{12}\right)}{\Gamma\left(\frac{l_{1}+l_{2}+2}{2}+\mathrm{i} u_{12}\right)}\right|^{2} \frac{\left[\mathbb{O}_{l_{1}, l_{2}}\left(u_{21}\right)^{t} \mathbb{O}_{l_{1}, l_{2}}\left(u_{12}\right) x^{\otimes\left(l_{1}+l_{2}\right)}\right] \cdot y^{\otimes\left(l_{1}+l_{2}\right)}}{(|x||y|)^{l_{1}+l_{2}}} .
\end{aligned}
$$

Notice that since $\mathbb{O}_{l_{1}, l_{2}}$ goes from $\mathbb{V}_{l_{1}} \otimes \mathbb{V}_{l_{2}}$ to $S^{l_{1}+l_{2}}\left(\mathbb{C}^{d}\right)$ we can only multiply it with its transpose. This is what happens here where we need matrix elements of $\mathbb{O}_{l_{1}, l_{2}}\left(u_{21}\right)^{t} \mathbb{O}_{l_{1}, l_{2}}\left(u_{12}\right): S^{l_{1}+l_{2}}\left(\mathbb{C}^{d}\right) \rightarrow S^{l_{1}+l_{2}}\left(\mathbb{C}^{d}\right)$. It seems that expression (4.19) is the most natural for the generalization to the general case $I_{M, N}^{(d, \delta)}$.

Let us compare the expressions (4.18) for integral $I_{M, 2}^{(d, \delta)}$ in various dimensions. The limit $d \rightarrow 2$ is seemingly singular but one should remember that the Gegenbauer polynomials for $l>0$ tend to 0 in this limit so that

$$
\forall l \in \mathbb{N}, \quad \frac{2 l+d-2}{d-2} C_{l}^{\left(\frac{d-2}{2}\right)}(\cos \theta) \underset{d \rightarrow 2}{\longrightarrow} \frac{2}{1+\delta_{l, 0}} \cos l \theta .
$$

Thus, for $\min \left(l_{1}, l_{2}\right)>0$, one has

$$
\frac{\left(2 l_{1}+d-2\right)\left(2 l_{2}+d-2\right)}{(d-2)^{2}} a_{l_{1}, l_{2}, m} C_{l_{1}+l_{2}-2 m}^{\left(\frac{d-2}{2}\right)}(\cos \theta) \underset{d \rightarrow 2}{\longrightarrow} 2\left[\delta_{m, 0} \cos \left(l_{1}+l_{2}\right) \theta+\delta_{m, \min \left(l_{1}, l_{2}\right)} \cos \left(l_{1}-l_{2}\right) \theta\right] .
$$

In the end, $I_{M, 2}^{(2, \delta)}$ is finite (as it should be) and, using the additional symmetry $Q_{l}=Q_{-l}$ valid for $l \in \mathbb{Z}$ when $d=2$, one can extend the sum to $\left(l_{1}, l_{2}\right) \in \mathbb{Z}^{2}$ so that

$$
I_{M, 2}^{(2, \delta)}(x, y)=\frac{1}{2\left(x^{2} y^{2}\right)^{\tilde{\delta}}} \sum_{\left(l_{1}, l_{2}\right) \in \mathbb{Z}^{2}} \mathrm{e}^{\mathrm{i}\left(l_{1}+l_{2}\right) \theta} \int \frac{\mathrm{d} u_{1} \mathrm{~d} u_{2}}{(2 \pi)^{2}}\left(\frac{x^{2}}{y^{2}}\right)^{\mathrm{i}\left(u_{1}+u_{2}\right)}\left[Q_{l_{1}}\left(u_{1}\right) Q_{l_{2}}\left(u_{2}\right)\right]^{M+2}\left[u_{12}^{2}+\frac{l_{12}^{2}}{4}\right] .
$$

This coincides with the result of [12]. 
When $d=4$, the dependence on $u$ of the sum over $m$ disappears and the sum is then simply

$$
C_{l_{1}}^{(1)}(\cos \theta) C_{l_{2}}^{(1)}(\cos \theta)=\frac{\left(\mathrm{e}^{\mathrm{i}\left(l_{1}+1\right) \theta}-\mathrm{e}^{-\mathrm{i}\left(l_{1}+1\right) \theta}\right)\left(\mathrm{e}^{\mathrm{i}\left(l_{2}+1\right) \theta}-\mathrm{e}^{-\mathrm{i}\left(l_{2}+1\right) \theta}\right)}{\left(\mathrm{e}^{\mathrm{i} \theta}-\mathrm{e}^{-\mathrm{i} \theta}\right)^{2}} .
$$

Noticing that $Q_{l}=Q_{-l-2}$ when $d=4$ we can keep only one of the four terms in the equation above if we extend the summation to $\left(l_{1}, l_{2}\right) \in \mathbb{Z}^{2}$. We thus recover the known formula [7] (we have also replaced $l_{j}$ with $a_{j}=l_{j}+1$ ):

$$
\begin{aligned}
& I_{M, 2}^{(4, \delta)}(x, y)=\frac{1}{2\left(x^{2} y^{2}\right)^{\tilde{\delta}}} \frac{1}{\left(\mathrm{e}^{\mathrm{i} \theta}-\mathrm{e}^{-\mathrm{i} \theta}\right)^{2}} \sum_{\left(a_{1}, a_{2}\right) \in \mathbb{Z}^{2}} a_{1} a_{2} \mathrm{e}^{\mathrm{i}\left(a_{1}+a_{2}\right) \theta} \\
& \times \int \frac{\mathrm{d} u_{1} \mathrm{~d} u_{2}}{(2 \pi)^{2}}\left(\frac{x^{2}}{y^{2}}\right)^{\mathrm{i}\left(u_{1}+u_{2}\right)}\left[Q_{a_{1}-1}\left(u_{1}\right) Q_{a_{2}-1}\left(u_{2}\right)\right]^{M+2}\left[u_{12}^{2}+\frac{a_{12}^{2}}{4}\right]\left[u_{12}^{2}+\frac{\left(a_{1}+a_{2}\right)^{2}}{4}\right] .
\end{aligned}
$$

The next case we could investigate is $(d, \delta)=(6,1)$, the formula $(4.18)$ then reads

$$
\begin{aligned}
I_{M, 2}^{(6,1)}(x, y) & \frac{1}{2\left(x^{2} y^{2}\right)^{2}} \sum_{l_{1}, l_{2}} \int \frac{\mathrm{d} u_{1} \mathrm{~d} u_{2}}{(2 \pi)^{2}}\left(\frac{x^{2}}{y^{2}}\right)^{\mathrm{i}\left(u_{1}+u_{2}\right)} \frac{\left[u_{12}^{2}+\frac{l_{12}^{2}}{4}\right]\left[u_{12}^{2}+\frac{\left(l_{1}+l_{2}+4\right)^{2}}{4}\right]}{\left[\left(u_{1}^{2}+\frac{\left(l_{1}+2\right)^{2}}{4}\right)\left(u_{2}^{2}+\frac{\left(l_{2}+2\right)^{2}}{4}\right)\right]^{M+2}}\left(l_{1}+2\right)\left(l_{2}+2\right) \\
& \sum_{m=0}^{\min \left(l_{1}, l_{2}\right)} \frac{(m+1)\left(l_{1}-m+1\right)\left(l_{2}-m+1\right)\left(l_{1}+l_{2}-m+3\right)}{\left(l_{1}+l_{2}-2 m+1\right)\left(l_{1}+l_{2}-2 m+3\right)}\left[u_{12}^{2}+\frac{\left(l_{1}+l_{2}-2 m+2\right)^{2}}{4}\right] C_{l_{1}+l_{2}-2 m}^{(2)}(\cos \theta) .
\end{aligned}
$$

The integrals are rather easy, at least when $M$ is not too large, but the sums seem to be quite tedious to perform. We hope that this example of the first nontrivial integral $I_{M, 2}^{(d, \delta)}$ clearly illustrates the complications arising in higher dimensions.

\section{Conclusions}

In the present paper, we have constructed the generalised eigenvectors of the graph-building operator for fishnet integrals in $d$ dimensions. The spectral decomposition of the graphbuilding operator allowed us to derive a representation for the $d$-dimensional Basso-Dixon diagrams in terms of separated variables, i.e. the rapidities $u_{j}$ and the bound-state numbers $l_{j}$ of the fishnet lattice's excitations. According to that, the expression for the Basso-Dixon diagram is an integral over separated variables - with the corresponding Sklyanin measure that we computed for any $d$ from the overlap of eigenvectors, and it reproduces the results of $[7,12,14]$ in two and four dimensions. The integrand is given by the eigenvalues of the graph-building operator and by the reductions of the bra and ket eigenvectors corresponding to pinching their external coordinates to two points $x$ and $y$. The former is factorised into $N$ contributions, each depending on the quantum numbers of one excitation $\left(u_{j}, l_{j}\right)$, the latter have a more complicated structure for general $d$. In $d=2$ and $d=4$, the eigenvectors 
are drastically simplified by the reduction, but, as we have demonstrated in the last section, the analogous expression for the general $d$-dimensional situation is more involved.

The present construction of eigenvectors is based on the symmetric tensor representations of the group $O(d)$. As a consequence, the corresponding main interchange relation governing the symmetry of the eigenvectors involves $O(d)$-invariant R-matrix acting on the tensor product of two symmetric tensor representations. In this framework, the symmetry of eigenvectors and scalar product look simple. On the other hand the special reduction of the eigenvector we would need in order to write the Basso-Dixon diagrams in terms of known functions is quite complicated.

In appendix (E) we have discussed shortly the construction of the eigenvectors based on spinor representations of the group $O(d)$. In some sense these constructions are complementary and show opposite features: in the spinorial framework the symmetry properties and scalar products looks more complicated but the special reductions of eigenvectors is straightforward. A detailed discussion of such duality, comparison between the spinorial and tensorial frameworks, and the derivation of the general expression for the reduced eigenvectors is left to a future paper.

Our results constitute an important step for a formulation of integrability techniques for $n$-point functions $(n \geqslant 3)$ in conformal field theories in dimension $d \neq 4$. This claim is based on the fact that techniques of hexagonalisation [56-58] developed in the 4-dimensional $\mathcal{N}=4 \mathrm{SYM}$ theory took an important piece information from the knowledge of Fishnet integrals, and can even be derived from first principle for the strongly deformed (Fishnet) theory $[59,60]$. At the same time, nothing is known about similar techniques in other dimensions, with the exception of a worldsheet theory without a known field theory dual [61]. For example, one can wonder if and how hexagon form factors and octagon functions [3, 4] can be computed in $\mathcal{N}=6$ ABJM theory: a rich piece of information would come from an explicit computation of BD diagrams in $3 D$ together with the discovery of an analogue of its representation as a determinant of ladder integrals, following the observations in $d=2,4[12,13]$.

\section{Acknowledgments}

We are grateful to the referees for the detailed read of our manuscript and for several valuable suggestions. G.F. thanks B. Basso and V. Kazakov for numerous fruitful discussions, and D. Serban and M. Staudacher for their many useful comments on an early version of the manuscript. Research at the Perimeter Institute is supported in part by the Government of Canada through NSERC and by the Province of Ontario through MRI. This work was additionally supported by a grant from the Simons Foundation (Simons Collaboration on the Nonperturbative Bootstrap).

\section{A Basic integral relations}

We collect in this appendix various formulae which are used for the calculations of the Feynman diagrams [62-67]. We recall that for a complex number $a$ and an integer $l \geqslant 0$ 


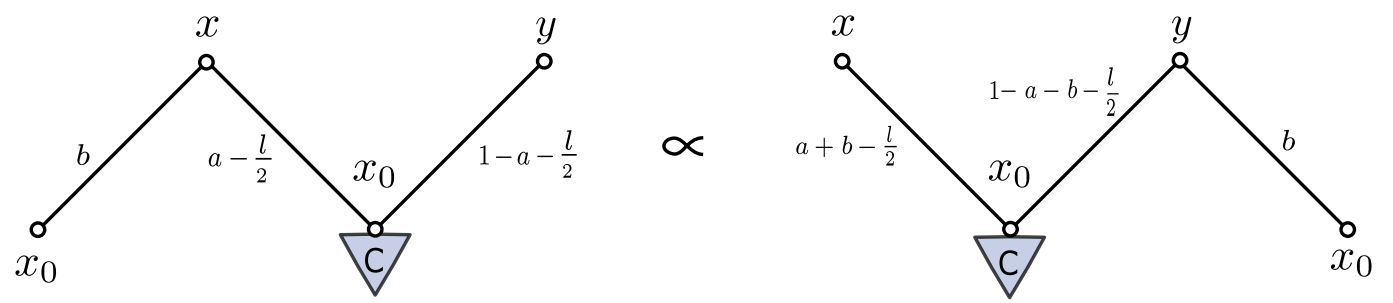

Figure 18. Graphical representation of the identity (A.5).

we define

$$
\tilde{a}=\frac{d}{2}-a, \quad A_{l}(a)=\frac{\Gamma\left(\tilde{a}+\frac{l}{2}\right)}{\Gamma\left(a+\frac{l}{2}\right)}=\frac{1}{A_{l}(\tilde{a})}, \quad(a)_{l}=\frac{\Gamma(a+l)}{\Gamma(a)}=\prod_{k=0}^{l-1}(a+k) .
$$

If $C$ is a symmetric traceless tensor of rank $l$, i.e. $C \in \mathbb{V}_{l}$, and $x \in \mathbb{R}^{d}$ we will also write

$$
C(x)=C^{\mu_{1} \cdots \mu_{l}} x_{\mu_{1}} \ldots x_{\mu_{l}} .
$$

Because $C$ is traceless the following two elementary but very useful properties hold for an arbitrary complex number $a$ :

$$
\frac{C\left(\frac{x-y}{|x-y|}\right)}{(x-y)^{2 a}}=\frac{C\left(\nabla_{x}\right)}{(-2)^{l}\left(a-\frac{l}{2}\right)_{l}} \frac{1}{(x-y)^{2\left(a-\frac{l}{2}\right)}}
$$

and

$$
\frac{C\left(\frac{x-x_{0}}{\left(x-x_{0}\right)^{2}}-\frac{y-x_{0}}{\left(y-x_{0}\right)^{2}}\right)}{\left(x-x_{0}\right)^{2\left(a-\frac{l}{2}\right)}\left(y-x_{0}\right)^{2\left(1-a-\frac{l}{2}\right)}}=\frac{C\left(\nabla_{x_{0}}\right)}{(-2)^{l}\left(a-\frac{l}{2}\right)_{l}} \frac{1}{\left(x-x_{0}\right)^{2\left(a-\frac{l}{2}\right)}\left(y-x_{0}\right)^{2\left(1-a-\frac{l}{2}\right)}} .
$$

The second property in particular implies that for arbitrary complex numbers $a$ and $b$ one has

$$
\begin{aligned}
& \left.\frac{1}{\left(x-x_{0}\right)^{2 b}} C\left(\nabla_{w_{0}}\right)\left[\frac{1}{\left(x-w_{0}\right)^{2\left(a-\frac{l}{2}\right)}\left(y-w_{0}\right)^{2\left(1-a-\frac{l}{2}\right)}}\right]\right|_{w_{0}=x_{0}} \\
& =\left.\frac{1}{\left(y-x_{0}\right)^{2 b}} \frac{\left(a-\frac{l}{2}\right)_{l}}{\left(a+b-\frac{l}{2}\right)_{l}} C\left(\nabla_{w_{0}}\right)\left[\frac{1}{\left(x-w_{0}\right)^{2\left(a+b-\frac{l}{2}\right)}\left(y-w_{0}\right)^{2\left(1-a-b-\frac{l}{2}\right)}}\right]\right|_{w_{0}=x_{0}} .
\end{aligned}
$$

This useful identity can be visualised in a graphic form in figure 18.

We also recall that, if $\Re(a)>0$, one can write

$$
\frac{1}{x^{2 a}}=\frac{1}{\Gamma(a)} \int_{0}^{+\infty} \mathrm{e}^{-u x^{2}} u^{a-1} \mathrm{~d} u .
$$

Fourier transform of a propagator. For $C$ a rank $l$ symmetric traceless tensor,

$$
\int \frac{C\left(\frac{p}{|p|}\right)}{p^{2 a}} \mathrm{e}^{\mathrm{i} p \cdot x} \frac{\mathrm{d}^{d} p}{\pi^{\frac{d}{2}}}=A_{l}(a) \mathrm{i}^{l} 4^{\tilde{a}} \frac{C\left(\frac{x}{|x|}\right)}{x^{2 \tilde{a}}} .
$$



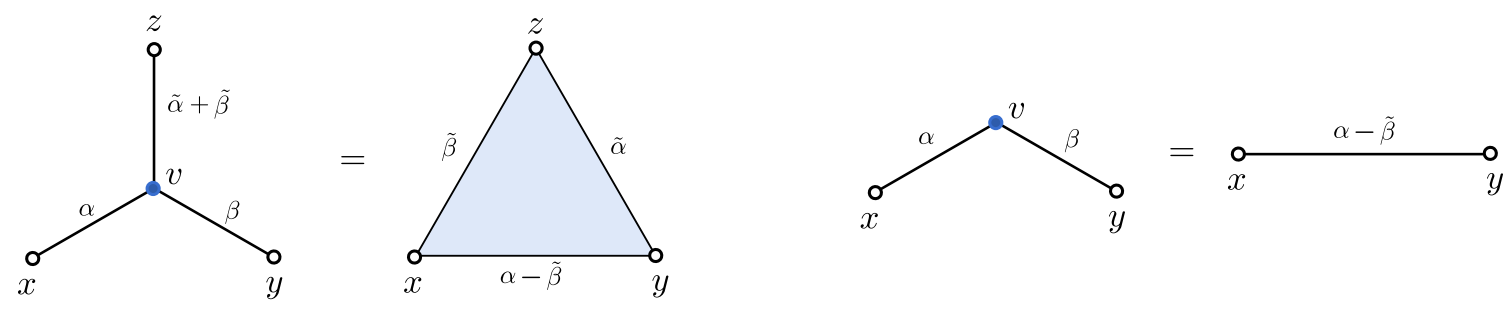

Figure 19. Left: star-triangle identity in graphical form. We recall that $\tilde{x}=\frac{d}{2}-x$. Right: chainrule identity in graphical form.
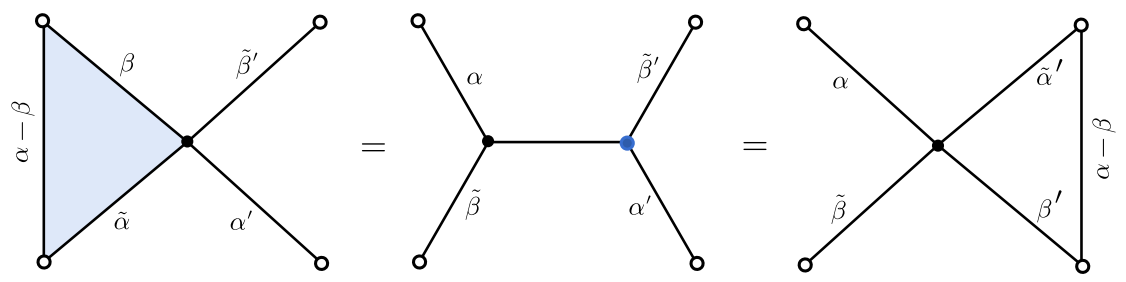

Figure 20. The exchange relation corresponding to the notation of blue arrow used in the main text amounts to consecutive triangle-star and star-triangle transformations, and holds under the assumption $\alpha+\beta^{\prime}=\alpha^{\prime}+\beta$. The equalities hold modulo numerical coefficients depending on $\alpha, \beta, \alpha^{\prime}, \beta^{\prime}$.

\section{Chain relation.}

$$
\int \frac{\pi^{-\frac{d}{2}} \mathrm{~d}^{d} z}{(x-z)^{2 a}(z-y)^{2 b}}=\frac{A_{0}(a) A_{0}(b) A_{0}(d-a-b)}{(x-y)^{2\left(a+b-\frac{d}{2}\right)}} .
$$

When $a+b=\frac{d}{2}$ the chain relation becomes

$$
\int \frac{\pi^{-\frac{d}{2}} \mathrm{~d}^{d} z}{(x-z)^{2 a}(z-y)^{2(d-a)}}=A_{0}(a) A_{0}(d-a) \pi^{\frac{d}{2}} \delta(x-y)
$$

Star-triangle relation. For $a+b+c=d$, one has

$$
\int \frac{\pi^{-\frac{d}{2}} \mathrm{~d}^{d} w}{(w-x)^{2 a}(w-y)^{2 b}(w-z)^{2 c}}=\frac{A_{0}(a) A_{0}(b) A_{0}(c)}{(x-y)^{2 \tilde{c}}(y-z)^{2 \tilde{a}}(z-x)^{2 \tilde{b}}} .
$$

Generalization of the chain relation. For $C \in \mathbb{V}_{l}$, one has

$$
\int \frac{C\left(\frac{x-z}{|x-z|}\right)}{(x-z)^{2 a}(z-y)^{2 b}} \frac{\mathrm{d}^{d} z}{\pi^{\frac{d}{2}}}=A_{l}(a) A_{0}(b) A_{l}(d-a-b) \frac{C\left(\frac{x-y}{|x-y|}\right)}{(x-y)^{2\left(a+b-\frac{d}{2}\right)}} .
$$




\section{B Equivalence (2.49) and (2.33)}

\section{B.1 Derivation of (2.50)}

In this section we derive representation (2.50) for the v-integral. For simplicity we shall use the compact notation $\lambda=1-\frac{l_{1}+l_{2}}{2}+\mathrm{i} u$. First step is the usual binomial expansion

$$
\begin{aligned}
\int \frac{\mathrm{d}^{d} v}{\pi^{\frac{d}{2}}} \frac{\left(\zeta \cdot\left(y-\frac{v}{v^{2}}\right)\right)^{l_{1}}\left(\eta \cdot\left(x-\frac{v}{v^{2}}\right)\right)^{l_{2}}}{(z-v)^{2(d-\lambda)} v^{2 \lambda}}= & \sum_{k, p}\left(\begin{array}{c}
l_{1} \\
k
\end{array}\right)\left(\begin{array}{c}
l_{2} \\
p
\end{array}\right)(-1)^{k+p} \\
& (\zeta \cdot y)^{l_{1}-k}(\eta \cdot x)^{l_{2}-p} \int \frac{\mathrm{d}^{d} v}{\pi^{\frac{d}{2}}} \frac{\left(\zeta \cdot \frac{v}{v^{2}}\right)^{k}\left(\eta \cdot \frac{v}{v^{2}}\right)^{p}}{(z-v)^{2(d-\lambda)} v^{2 \lambda}}
\end{aligned}
$$

On the second step we use representation

$$
\frac{\left(\zeta \cdot \frac{v}{v^{2}}\right)^{k}\left(\eta \cdot \frac{v}{v^{2}}\right)^{p}}{v^{2 \lambda}}=\left.\frac{1}{2^{k+p}(\lambda)_{k+p}} \partial_{t}^{k} \partial_{s}^{p} \frac{1}{\left(v^{2}-2 t \zeta \cdot v-2 s \eta \cdot v\right)^{\lambda}}\right|_{t=s=0}
$$

where the symbol $\left.\right|_{t=s=0}$ means that we have to put $t=0$ and $s=0$ after differentiation and series expansion $\left(\zeta^{2}=0\right.$ and $\left.\eta^{2}=0\right)$

$$
\frac{1}{\left(v^{2}-2 t \zeta \cdot v-2 s \eta \cdot v\right)^{\lambda}}=\frac{1}{\left((v-t \zeta-s \eta)^{2}-2 t s \zeta \cdot \eta\right)^{\lambda}}=\sum_{n} \frac{(\lambda)_{n}}{n !} \frac{(2 t s \zeta \cdot \eta)^{n}}{(v-t \zeta-s \eta)^{2(\lambda+n)}}
$$

to reduce our expression to the sum of the simpler integrals

$$
\begin{aligned}
& \int \frac{\mathrm{d}^{d} v}{\pi^{\frac{d}{2}}} \frac{\left(\zeta \cdot\left(y-\frac{v}{v^{2}}\right)\right)^{l_{1}}\left(\eta \cdot\left(x-\frac{v}{v^{2}}\right)\right)^{l_{2}}}{(z-v)^{2(d-\lambda)} v^{2 \lambda}} \\
& =\sum_{n, k, p}\left(\begin{array}{c}
l_{1} \\
k
\end{array}\right)\left(\begin{array}{c}
l_{2} \\
p
\end{array}\right)(-1)^{k+p} \frac{2^{n-k-p}(\lambda)_{n}}{n !(\lambda)_{k+p}}(\zeta \cdot y)^{l_{1}-k}(\eta \cdot x)^{l_{2}-p}(\zeta \cdot \eta)^{n} \\
& \left.\partial_{t}^{k} \partial_{s}^{p} t^{n} s^{n} \int \frac{\mathrm{d}^{d} v}{\pi^{\frac{d}{2}}} \frac{1}{(z-v)^{2(d-\lambda)}(v-t \zeta-s \eta)^{2(\lambda+n)}}\right|_{t=s=0}
\end{aligned}
$$

Next step we reduce remaining integral to the standard form

$$
\int \frac{\mathrm{d}^{d} v}{\pi^{\frac{d}{2}}} \frac{1}{v^{2(d-\lambda)}(v+z-t \zeta-s \eta)^{2 \lambda}}=\pi^{\frac{d}{2}} \frac{\Gamma\left(\lambda-\frac{d}{2}\right) \Gamma\left(\frac{d}{2}-\lambda\right)}{\Gamma(d-\lambda) \Gamma(\lambda)} \delta^{(d)}(z-t \zeta-s \eta)
$$


using external derivatives

$$
\begin{aligned}
\left(\partial_{z_{\mu}} \partial_{z_{\mu}}\right)^{n} \frac{1}{\left(z+z_{0}\right)^{2 \lambda}} & =4^{n}(\lambda)_{n}\left(\lambda-\frac{d}{2}+1\right)_{n} \frac{1}{\left(z+z_{0}\right)^{2(\lambda+n)}} \\
& \int \frac{\mathrm{d}^{d} v}{\pi^{\frac{d}{2}}} \frac{1}{v^{2(d-\lambda)}(v+z-t \zeta-s \eta)^{2(\lambda+n)}} \\
& =\frac{\left(\partial_{z_{\mu}} \partial_{z_{\mu}}\right)^{n}}{4^{n}(\lambda)_{n}\left(\lambda-\frac{d}{2}+1\right)_{n}} \int \frac{\mathrm{d}^{d} v}{\pi^{\frac{d}{2}}} \frac{1}{v^{2(d-\lambda)}(v+z-t \zeta-s \eta)^{2 \lambda}} \\
& =\frac{\pi^{\frac{d}{2}} \Gamma\left(\lambda-\frac{d}{2}\right) \Gamma\left(\frac{d}{2}-\lambda\right)}{4^{n}(\lambda)_{n}\left(\lambda-\frac{d}{2}+1\right)_{n} \Gamma(d-\lambda) \Gamma(\lambda)}\left(\partial_{z_{\mu}} \partial_{z_{\mu}}\right)^{n} \delta^{(d)}(z-t \zeta-s \eta)
\end{aligned}
$$

We have

$$
\begin{aligned}
\int \frac{\mathrm{d}^{d} v}{\pi^{\frac{d}{2}}} \frac{\left(\zeta \cdot\left(y-\frac{v}{v^{2}}\right)\right)^{l_{1}}\left(\eta \cdot\left(x-\frac{v}{v^{2}}\right)\right)^{l_{2}}}{(z-v)^{2(d-\lambda)} v^{2 \lambda}} \\
=\sum_{n, k, p}\left(\begin{array}{c}
l_{1} \\
k
\end{array}\right)\left(\begin{array}{c}
l_{2} \\
p
\end{array}\right)(-1)^{k+p} \frac{2^{-n-k-p} \pi^{\frac{d}{2}} \Gamma\left(\lambda-\frac{d}{2}\right) \Gamma\left(\frac{d}{2}-\lambda\right)}{n !(\lambda)_{k+p}\left(\lambda-\frac{d}{2}+1\right)_{n} \Gamma(d-\lambda) \Gamma(\lambda)} \\
\left.\quad(\zeta \cdot y)^{l_{1}-k}(\eta \cdot x)^{l_{2}-p}(\zeta \cdot \eta)^{n} \partial_{t}^{k} \partial_{s}^{p} t^{n} s^{n}\left(\partial_{z_{\mu}} \partial_{z_{\mu}}\right)^{n} \delta^{(d)}(z-t \zeta-s \eta)\right|_{t=s=0}
\end{aligned}
$$

The last transformation: using evident formula

$$
\left.\partial_{t}^{k} t^{n} F(t)\right|_{t=0}=\left.\frac{k !}{(k-n) !} \partial_{t}^{k-n} F(t)\right|_{t=0}
$$

and similar ones for s-derivative and shifting summation indices $k \rightarrow k+n$ and $p \rightarrow p+n$ one obtains

$$
\begin{aligned}
& \int \frac{\mathrm{d}^{d} v}{\pi^{\frac{d}{2}}} \frac{\left(\zeta \cdot\left(y-\frac{v}{v^{2}}\right)\right)^{l_{1}}\left(\eta \cdot\left(x-\frac{v}{v^{2}}\right)\right)^{l_{2}}}{(z-v)^{2(d-\lambda)} v^{2 \lambda}} \\
= & \frac{\pi^{\frac{d}{2}} \Gamma\left(\lambda-\frac{d}{2}\right) \Gamma\left(\frac{d}{2}-\lambda\right)}{\Gamma(d-\lambda) \Gamma(\lambda)} \sum_{n, k, p}\left(\begin{array}{c}
l_{1} \\
k+n
\end{array}\right)\left(\begin{array}{c}
l_{2} \\
p+n
\end{array}\right) \frac{(k+n) !(p+n) !}{k ! p !} \frac{2^{-3 n-k-p}(-1)^{k+p}}{n !(\lambda)_{k+p+2 n}\left(\lambda-\frac{d}{2}+1\right)_{n}} \\
& \left.(\zeta \cdot y)^{l_{1}-k-n}(\eta \cdot x)^{l_{2}-p-n}(\zeta \cdot \eta)^{n} \partial_{t}^{k} \partial_{s}^{p}\left(\partial_{z_{\mu}} \partial_{z_{\mu}}\right)^{n} \delta^{(d)}(z-t \zeta-s \eta)\right|_{t=s=0}
\end{aligned}
$$

It is exactly expression (2.50) and

$$
A_{l_{1}, l_{2}}(u)=\frac{\pi^{\frac{d}{2}} \Gamma\left(\lambda-\frac{d}{2}\right) \Gamma\left(\frac{d}{2}-\lambda\right)}{\Gamma(d-\lambda) \Gamma(\lambda)} ; \lambda=1-\frac{l_{1}+l_{2}}{2}+\mathrm{i} u
$$




\section{B.2 Equivalence}

Now we are going to calculate sum in the right hand side of (2.51) and let us continue to use for simplicity notation $\lambda=1-\frac{l_{1}+l_{2}}{2}+\mathrm{i} u$. We have

$$
\begin{aligned}
& F_{l_{1}, l_{2}}(u)=\frac{\Gamma\left(2-\lambda-l_{1}-l_{2}\right) \Gamma\left(\lambda+l_{1}+l_{2}\right) \Gamma(d-\lambda)}{\Gamma(2-\lambda) \Gamma\left(\frac{d}{2}-\lambda\right) \Gamma\left(\lambda-\frac{d}{2}\right)}, \quad A_{l_{1}, l_{2}}(u)=\frac{\pi^{\frac{d}{2}} \Gamma\left(\lambda-\frac{d}{2}\right) \Gamma\left(\frac{d}{2}-\lambda\right)}{\Gamma(d-\lambda) \Gamma(\lambda)}, \\
& \pi^{-\frac{d}{2}} F_{l_{1}, l_{2}}(u) A_{l_{1}, l_{2}}(u)=\frac{\Gamma\left(2-\lambda-l_{1}-l_{2}\right) \Gamma\left(\lambda+l_{1}+l_{2}\right)}{\Gamma(2-\lambda) \Gamma(\lambda)}=(-1)^{l_{1}+l_{2}} \frac{\lambda-1+l_{1}+l_{2}}{\lambda-1}
\end{aligned}
$$

so that

$$
\begin{aligned}
& {\left[\mathbb{R}_{l_{1}, l_{2}}(u) \zeta^{\otimes l_{1}} \otimes \eta^{\otimes l_{2}}\right] \cdot x^{\otimes l_{1}} \otimes y^{\otimes l_{2}}} \\
& =\frac{\Gamma\left(2-\lambda-l_{1}-l_{2}\right) \Gamma\left(\lambda+l_{1}+l_{2}\right)}{\Gamma(2-\lambda) \Gamma(\lambda)} \\
& \quad \sum_{n, k, p} \frac{l_{1} ! l_{2} !(-1)^{k+p} 2^{-k-p-3 n}}{\left(l_{1}-k-n\right) !\left(l_{2}-p-n\right) ! k ! p ! n !} \frac{(\zeta \cdot \eta)^{n}(\zeta \cdot y)^{l_{1}-k-n}(\eta \cdot x)^{l_{2}-p-n}}{(\lambda)_{k+p+2 n}\left(\lambda-\frac{d}{2}+1\right)_{n}} \\
& \left.\quad \partial_{t}^{k} \partial_{s}^{p}\left(\partial_{z_{\mu}} \partial_{z_{\mu}}\right)^{n}\left(1-2 z \cdot x+z^{2} x^{2}\right)^{1-\lambda-l_{2}}\left(1-2 z \cdot y+z^{2} y^{2}\right)^{1-\lambda-l_{1}}\right|_{z=t \zeta+s \eta}
\end{aligned}
$$

The symbol $\left.\right|_{z=t \zeta+s \eta}$ means that we have to substitute $z=t \zeta+s \eta$ after all z-differentiation and then to put $t=s=0$ after all s- and t-differentiations.

The first step is the calculation of the expression in the last line. We introduce Schwinger parameters

$$
\begin{aligned}
& \left(1-2 z \cdot x+z^{2} x^{2}\right)^{1-\lambda-l_{2}}\left(1-2 z \cdot y+z^{2} y^{2}\right)^{1-\lambda-l_{1}} \\
& =\frac{1}{\Gamma\left(\lambda-1+l_{2}\right) \Gamma\left(\lambda-1+l_{1}\right)} \int_{0}^{\infty} \mathrm{d} \alpha \alpha^{\lambda-2+l_{2}} e^{-\alpha} \int_{0}^{\infty} \mathrm{d} \beta \beta^{\lambda-2+l_{1}} e^{-\beta} e^{2 z \cdot(\alpha x+\beta y)-z^{2}\left(\alpha x^{2}+\beta y^{2}\right)}
\end{aligned}
$$

and then calculate z-derivatives using formula

$$
e^{\gamma \partial_{z_{\mu}} \partial_{z_{\mu}}} e^{-b z^{2}+z \cdot c}=(1+4 \gamma b)^{-\frac{d}{2}} e^{\frac{\gamma c^{2}}{1+4 \gamma b}} e^{\frac{-b z^{2}+z \cdot c}{1+4 \gamma b}}
$$

This formula can be easily obtained using Gaussian integral and in our case $b=\alpha x^{2}+\beta y^{2}$ and $c=2(\alpha x+\beta y)$. We have

$$
\begin{aligned}
& \left(\partial_{z_{\mu}} \partial_{z_{\mu}}\right)^{n} e^{-b z^{2}+z \cdot c} \\
& =\left.\partial_{\gamma}^{n}(1+4 \gamma b)^{-\frac{d}{2}} e^{\frac{\gamma c^{2}}{1+4 \gamma b}} e^{\frac{-b z^{2}+z \cdot c}{1+4 \gamma b}}\right|_{\gamma=0} \\
& =\left.4^{n} b^{n} \partial_{\gamma}^{n}(1+\gamma)^{-\frac{d}{2}} e^{\frac{\gamma}{1+\gamma} \frac{c^{2}}{4 b}} e^{\frac{-b z^{2}+z \cdot c}{1+\gamma}}\right|_{\gamma=0} \\
& =\left.\sum_{m} \frac{1}{m !} \partial_{\gamma}^{n} \gamma^{m}(1+\gamma)^{-\frac{d}{2}-m} 4^{n-m} b^{n-m}\left(c^{2}\right)^{m} e^{\frac{-b z^{2}+z \cdot c}{1+\gamma}}\right|_{\gamma=0}
\end{aligned}
$$


Now it is possible to substitute $z=t \zeta+s \eta$ so that $z^{2}=2 t s \zeta \cdot \eta$ and $z \cdot c=t \zeta \cdot c+s \eta \cdot c$ and calculate t- and s-derivatives:

$$
\begin{aligned}
& \left.\partial_{t}^{k} \partial_{s}^{p} e^{\frac{-2 t s b \zeta \cdot \eta+t \zeta \cdot c+s \eta \cdot c}{1+\gamma}}\right|_{t=s=0} \\
& =\left.(1+\gamma)^{-k} \partial_{s}^{p}(-2 s b \zeta \cdot \eta+\zeta \cdot c)^{k} e^{\frac{s \eta \cdot c}{1+\gamma}}\right|_{s=0} \\
& =\left.(1+\gamma)^{-k} \sum_{m_{1}}\left(\begin{array}{c}
k \\
m_{1}
\end{array}\right)(-2 b \zeta \cdot \eta)^{m_{1}}(\zeta \cdot c)^{k-m_{1}} \partial_{s}^{p} s^{m_{1}} e^{\frac{s \eta \cdot c}{1+\gamma}}\right|_{s=0} \\
& =\sum_{m_{1}}\left(\begin{array}{c}
k \\
m_{1}
\end{array}\right) \frac{p !}{\left(p-m_{1}\right) !}(1+\gamma)^{-k-p+m_{1}}(-2 b \zeta \cdot \eta)^{m_{1}}(\zeta \cdot c)^{k-m_{1}}(\eta \cdot c)^{p-m_{1}}
\end{aligned}
$$

Using formula for $\gamma$-derivative

$$
\left.\partial_{\gamma}^{n} \gamma^{m}(1+\gamma)^{-\frac{d}{2}-m-k-p+m_{1}}\right|_{\gamma=0}=(-1)^{n-m} \frac{n !\left(\frac{d}{2}+m+k+p-m_{1}\right)_{n-m}}{(n-m) !}
$$

and collecting all terms we obtain

$$
\begin{aligned}
\left.\partial_{t}^{k} \partial_{s}^{p}\left(\partial_{z_{\mu}} \partial_{z_{\mu}}\right)^{n} e^{-b z^{2}+z \cdot c}\right|_{z=t \zeta+s \eta} \\
=\sum_{m, m_{1}}(-1)^{n-m+m_{1}} \frac{n ! k ! p !\left(\frac{d}{2}+m+k+p-m_{1}\right)_{n-m}}{m ! m_{1} !(n-m) !\left(k-m_{1}\right) !\left(p-m_{1}\right) !} 4^{n-m_{1}} 2^{m_{1}} \\
b^{n-m+m_{1}}\left(c^{2}\right)^{m}(\zeta \cdot \eta)^{m_{1}}(\zeta \cdot c)^{k-m_{1}}(\eta \cdot c)^{p-m_{1}}
\end{aligned}
$$

The symbol $\left.\right|_{z=t \zeta+s \eta}$ here and below means that we have to substitute $z=t \zeta+s \eta$ after all z-differentiation and then to put $t=s=0$ after all s- and t-differentiations.

Let us return to our calculation. We have

$$
\begin{aligned}
& \left.\partial_{t}^{k} \partial_{s}^{p}\left(\partial_{z_{\mu}} \partial_{z_{\mu}}\right)^{n} e^{2 z \cdot(\alpha x+\beta y)-z^{2}\left(\alpha x^{2}+\beta y^{2}\right)}\right|_{z=t \zeta+s \eta} \\
& =\sum_{m, m_{1}}(-1)^{n-m+m_{1}} \frac{n ! k ! p !\left(\frac{d}{2}+m+k+p-m_{1}\right)_{n-m}}{m ! m_{1} !(n-m) !\left(k-m_{1}\right) !\left(p-m_{1}\right) !} 4^{n} 2^{k+p-m_{1}}(\zeta \cdot \eta)^{m_{1}} \\
& \quad\left(\alpha x^{2}+\beta y^{2}\right)^{n-m+m_{1}}\left(\alpha^{2} x^{2}+2 \alpha \beta x \cdot y+\beta^{2} y^{2}\right)^{m}(\alpha \zeta \cdot x+\beta \zeta \cdot y)^{k-m_{1}}(\alpha \eta \cdot x+\beta \eta \cdot y)^{p-m_{1}}
\end{aligned}
$$

so that it remains to use binomial expansions

$$
\begin{aligned}
\left(\alpha x^{2}+\beta y^{2}\right)^{n-m+m_{1}} & =\sum_{k_{1}}\left(\begin{array}{c}
n-m+m_{1} \\
k_{1}
\end{array}\right) \alpha^{k_{1}} \beta^{n-m+m_{1}-k_{1}}\left(x^{2}\right)^{k_{1}}\left(y^{2}\right)^{n-m+m_{1}-k_{1}}, \\
(\alpha \zeta \cdot x+\beta \zeta \cdot y)^{k-m_{1}} & =\sum_{k_{2}}\left(\begin{array}{c}
k-m_{1} \\
k_{2}
\end{array}\right) \alpha^{k_{2}} \beta^{k-m_{1}-k_{2}}(\zeta \cdot x)^{k_{2}}(\zeta \cdot y)^{k-m_{1}-k_{2}}, \\
(\alpha \eta \cdot x+\beta \eta \cdot y)^{p-m_{1}} & =\sum_{k_{3}}\left(\begin{array}{c}
p-m_{1} \\
k_{3}
\end{array}\right) \alpha^{k_{3}} \beta^{p-m_{1}-k_{3}}(\eta \cdot x)^{k_{3}}(\eta \cdot y)^{p-m_{1}-k_{3}}, \\
\left(\alpha^{2} x^{2}+2 \alpha \beta x \cdot y+\beta^{2} y^{2}\right)^{m} & =\sum_{s_{1}, s_{2}} \frac{2^{s_{2}} m ! \alpha^{2 s_{1}+s_{2}} \beta^{2 m-2 s_{1}-s_{2}}}{\left(m-s_{1}-s_{2}\right) ! s_{1} ! s_{2} !}\left(x^{2}\right)^{s_{1}}(x \cdot y)^{s_{2}}\left(y^{2}\right)^{m-s_{1}-s_{2}}
\end{aligned}
$$


and then calculate $\alpha$ - and $\beta$-integrals

$$
\begin{aligned}
& \frac{1}{\Gamma\left(\lambda-1+l_{2}\right)} \int_{0}^{\infty} \mathrm{d} \alpha \alpha^{\lambda-2+l_{2}} \alpha^{L} e^{-\alpha}=\left(\lambda-1+l_{2}\right)_{L} \\
& \frac{1}{\Gamma\left(\lambda-1+l_{1}\right)} \int_{0}^{\infty} \mathrm{d} \beta \beta^{\lambda-2+l_{1}} \beta^{n+k+p+m-L-m_{1}} e^{-\beta}=\left(\lambda-1+l_{1}\right)_{n+k+p+m-L-m_{1}}
\end{aligned}
$$

where for simplicity we denote $L=k_{1}+k_{2}+k_{3}+2 s_{1}+s_{2}$. Collecting all pieces together we obtain the following intermediate result

$$
\begin{aligned}
\left.\partial_{t}^{k} \partial_{s}^{p}\left(\partial_{z_{\mu}} \partial_{z_{\mu}}\right)^{n}\left(1-2 z \cdot x+z^{2} x^{2}\right)^{1-\lambda-l_{2}}\left(1-2 z \cdot y+z^{2} y^{2}\right)^{1-\lambda-l_{1}}\right|_{z=t \zeta+s \eta} \\
=\sum \frac{2^{2 n+k+p-m_{1}+s_{2}}(-1)^{n-m+m_{1}} n ! k ! p !\left(n-m+m_{1}\right) !}{m_{1} !(n-m) !\left(n-m+m_{1}-k_{1}\right) !\left(k-m_{1}-k_{2}\right) !\left(p-m_{1}-k_{3}\right) !\left(m-s_{1}-s_{2}\right) ! k_{1} ! k_{2} ! k_{3} ! s_{1} ! s_{2} !} \\
\quad\left(\frac{d}{2}+m+k+p-m_{1}\right)_{n-m}\left(\lambda-1+l_{2}\right)_{L}\left(\lambda-1+l_{1}\right)_{n+k+p+m-L-m_{1}} \\
\quad\left(x^{2}\right)^{k_{1}+s_{1}}\left(y^{2}\right)^{n+m_{1}-k_{1}-s_{1}-s_{2}}(\zeta \cdot \eta)^{m_{1}}(x \cdot y)^{s_{2}}(\zeta \cdot x)^{k_{2}}(\zeta \cdot y)^{k-m_{1}-k_{2}}(\eta \cdot x)^{k_{3}}(\eta \cdot y)^{p-m_{1}-k_{3}}
\end{aligned}
$$

where summation is performed over $m, m_{1}, k_{1}, k_{2}, k_{3}, s_{1}, s_{2}$ and for simplicity we do not show it explicitly. Substitution of this expression in B.7 gives

$$
\begin{aligned}
& {\left[\mathbb{R}_{l_{1}, l_{2}}(u) \zeta^{\otimes l_{1}} \otimes \eta^{\otimes l_{2}}\right] \cdot x^{\otimes l_{1}} \otimes y^{\otimes l_{2}} } \\
= & \frac{\Gamma\left(2-\lambda-l_{1}-l_{2}\right) \Gamma\left(\lambda+l_{1}+l_{2}\right)}{\Gamma(2-\lambda) \Gamma(\lambda)} \\
& \sum_{n, k, p} \frac{l_{1} ! l_{2} !(-1)^{k+p}}{\left(l_{1}-k-n\right) !\left(l_{2}-p-n\right) !} \frac{1}{(\lambda)_{k+p+2 n}\left(\lambda-\frac{d}{2}+1\right)_{n}} \\
& \sum_{\frac{(-1)^{n-m+m_{1}}\left(n-m+m_{1}\right) !}{m_{1} !(n-m) !\left(n-m+m_{1}-k_{1}\right) !\left(k-m_{1}-k_{2}\right) !\left(p-m_{1}-k_{3}\right) !\left(m-s_{1}-s_{2}\right) ! k_{1} ! k_{2} ! k_{3} ! s_{1} ! s_{2} !}} \\
& \left(\frac{d}{2}+m+k+p-m_{1}\right)_{n-m}\left(\lambda-1+l_{2}\right)_{L}\left(\lambda-1+l_{1}\right)_{n+k+p+m-L-m_{1}} \\
& \left(\frac{x^{2}}{2}\right)^{k_{1}+s_{1}}\left(\frac{y^{2}}{2}\right)^{n+m_{1}-k_{1}-s_{1}-s_{2}}(\zeta \cdot \eta)^{n+m_{1}}(x \cdot y)^{s_{2}} \\
& (\zeta \cdot x)^{k_{2}}(\zeta \cdot y)^{l_{1}-n-m_{1}-k_{2}}(\eta \cdot x)^{l_{2}-p-n+k_{3}}(\eta \cdot y)^{p-m_{1}-k_{3}}
\end{aligned}
$$

Now we are going to show that some sequence of resummations allows to transform this expression to the form (2.33). We shall use two variants of Gauss summation formula

$$
\begin{aligned}
\sum_{k}\left(\begin{array}{l}
l \\
k
\end{array}\right)(-1)^{k} \frac{\Gamma(A+k)}{\Gamma(B+k)} & =\frac{\Gamma(A) \Gamma(B-A+l)}{\Gamma(B-A) \Gamma(B+l)} \\
\sum_{k}\left(\begin{array}{l}
l \\
k
\end{array}\right) \frac{1}{\Gamma(A+k) \Gamma(B-k)} & =\frac{\Gamma(A+B+l-1)}{\Gamma(B) \Gamma(A+B-1) \Gamma(A+l)}
\end{aligned}
$$


The first step: transformation $p \rightarrow p+m_{1}, k \rightarrow k+m_{1}$ and $n \rightarrow n-m_{1}$

$$
\begin{aligned}
& \sum \frac{l_{1} ! l_{2} !(-1)^{k+p}}{\left(l_{1}-k-n\right) !\left(l_{2}-p-n\right) !} \frac{1}{(\lambda)_{k+p+2 n}\left(\lambda-\frac{d}{2}+1\right)_{n-m_{1}}} \\
& \frac{(-1)^{n-m}(n-m) !}{m_{1} !\left(n-m-m_{1}\right) !\left(n-m-k_{1}\right) !\left(k-k_{2}\right) !\left(p-k_{3}\right) !\left(m-s_{1}-s_{2}\right) ! k_{1} ! k_{2} ! k_{3} ! s_{1} ! s_{2} !} \\
& \left(\frac{d}{2}+m+k+p+m_{1}\right)_{n-m-m_{1}}\left(\lambda-1+l_{2}\right)_{L}\left(\lambda-1+l_{1}\right)_{n+k+p+m-L} \\
& \left(\frac{x^{2}}{2}\right)^{k_{1}+s_{1}}\left(\frac{y^{2}}{2}\right)^{n-k_{1}-s_{1}-s_{2}}(\zeta \cdot \eta)^{n}(x \cdot y)^{s_{2}}(\zeta \cdot x)^{k_{2}}(\zeta \cdot y)^{l_{1}-n-k_{2}}(\eta \cdot x)^{l_{2}-p-n+k_{3}}(\eta \cdot y)^{p-k_{3}}
\end{aligned}
$$

and then summation over $m_{1}$ using B.11

$$
\sum_{m_{1}} \frac{(n-m) !}{m_{1} !\left(n-m-m_{1}\right) !} \frac{\left(\frac{d}{2}+m+k+p+m_{1}\right)_{n-m-m_{1}}}{\left(\lambda-\frac{d}{2}+1\right)_{n-m_{1}}}=\frac{(\lambda)_{k+p+2 n}}{(\lambda)_{k+p+n+m}\left(\lambda-\frac{d}{2}+1\right)_{n}}
$$

leads to expression

$$
\begin{aligned}
& \sum \frac{l_{1} ! l_{2} !(-1)^{k+p}}{\left(l_{1}-k-n\right) !\left(l_{2}-p-n\right) !} \frac{1}{(\lambda)_{k+p+n+m}\left(\lambda-\frac{d}{2}+1\right)_{n}} \\
& \frac{(-1)^{n-m}}{\left(n-m-k_{1}\right) !\left(k-k_{2}\right) !\left(p-k_{3}\right) !\left(m-s_{1}-s_{2}\right) ! k_{1} ! k_{2} ! k_{3} ! s_{1} ! s_{2} !} \\
& \left(\lambda-1+l_{2}\right)_{L}\left(\lambda-1+l_{1}\right)_{n+k+p+m-L} \\
& \left(\frac{x^{2}}{2}\right)^{k_{1}+s_{1}}\left(\frac{y^{2}}{2}\right)^{n-k_{1}-s_{1}-s_{2}}(\zeta \cdot \eta)^{n}(x \cdot y)^{s_{2}}(\zeta \cdot x)^{k_{2}}(\zeta \cdot y)^{l_{1}-n-k_{2}}(\eta \cdot x)^{l_{2}-p-n+k_{3}}(\eta \cdot y)^{p-k_{3}}
\end{aligned}
$$

Now it is possible to perform summation over $m$ using B.10

$$
\begin{aligned}
& \sum_{m} \frac{(-1)^{m}\left(\lambda-1+l_{1}\right)_{n+k+p+m-L}}{\left(n-m-k_{1}\right) !\left(m-s_{1}-s_{2}\right) !(\lambda)_{k+p+n+m}} \\
& =\frac{(-1)^{s_{1}+s_{2}}\left(k_{2}+k_{3}+n+s_{1}-l_{1}\right) !\left(\lambda-1+l_{1}\right)_{n+k+p-k_{1}-k_{2}-k_{3}-s_{1}}}{\left(n-k_{1}-s_{1}-s_{2}\right) !\left(L-l_{1}\right) !(\lambda)_{k+p+2 n-k_{1}}}
\end{aligned}
$$

so that one obtains

$$
\begin{aligned}
& \sum \frac{l_{1} ! l_{2} !(-1)^{k+p}}{\left(l_{1}-k-n\right) !\left(l_{2}-p-n\right) !} \frac{1}{(\lambda)_{k+p+2 n-k_{1}}\left(\lambda-\frac{d}{2}+1\right)_{n}} \\
& \frac{(-1)^{n+s_{1}+s_{2}}\left(k_{2}+k_{3}+n+s_{1}-l_{1}\right) !}{\left(n-k_{1}-s_{1}-s_{2}\right) !\left(L-l_{1}\right) !\left(k-k_{2}\right) !\left(p-k_{3}\right) ! k_{1} ! k_{2} ! k_{3} ! s_{1} ! s_{2} !} \\
& \left(\lambda-1+l_{2}\right)_{L}\left(\lambda-1+l_{1}\right)_{n+k+p-k_{1}-k_{2}-k_{3}-s_{1}} \\
& \left(\frac{x^{2}}{2}\right)^{k_{1}+s_{1}}\left(\frac{y^{2}}{2}\right)^{n-k_{1}-s_{1}-s_{2}}(\zeta \cdot \eta)^{n}(x \cdot y)^{s_{2}}(\zeta \cdot x)^{k_{2}}(\zeta \cdot y)^{l_{1}-n-k_{2}}(\eta \cdot x)^{l_{2}-p-n+k_{3}}(\eta \cdot y)^{p-k_{3}}
\end{aligned}
$$


The second step: transformation $k_{1} \rightarrow k_{1}-s_{1}$ and $p \rightarrow p+k_{3}$

$$
\begin{aligned}
& \sum \frac{l_{1} ! l_{2} !(-1)^{k+p+k_{3}}}{\left(l_{1}-k-n\right) !\left(l_{2}-p-n-k_{3}\right) !} \frac{1}{(\lambda)_{k+p+2 n-k_{1}+s_{1}+k_{3}}\left(\lambda-\frac{d}{2}+1\right)_{n}} \\
& \frac{(-1)^{n+s_{1}+s_{2}}\left(k_{2}+k_{3}+n+s_{1}-l_{1}\right) !}{\left(n-k_{1}-s_{2}\right) !\left(k_{1}+k_{2}+k_{3}+s_{1}+s_{2}-l_{1}\right) !\left(k-k_{2}\right) ! p !\left(k_{1}-s_{1}\right) ! k_{2} ! k_{3} ! s_{1} ! s_{2} !} \\
& \left(\lambda-1+l_{2}\right)_{k_{1}+k_{2}+k_{3}+s_{1}+s_{2}}\left(\lambda-1+l_{1}\right)_{n+k+p-k_{1}-k_{2}} \\
& \left(\frac{x^{2}}{2}\right)^{k_{1}}\left(\frac{y^{2}}{2}\right)^{n-k_{1}-s_{2}}(\zeta \cdot \eta)^{n}(x \cdot y)^{s_{2}}(\zeta \cdot x)^{k_{2}}(\zeta \cdot y)^{l_{1}-n-k_{2}}(\eta \cdot x)^{l_{2}-p-n}(\eta \cdot y)^{p}
\end{aligned}
$$

then transformation $k_{3} \rightarrow k_{3}-s_{1}$

$$
\begin{aligned}
& \sum \frac{l_{1} ! l_{2} !(-1)^{k+p+k_{3}}}{\left(l_{1}-k-n\right) !\left(l_{2}-p-n-k_{3}+s_{1}\right) !} \frac{1}{(\lambda)_{k+p+2 n-k_{1}+k_{3}}\left(\lambda-\frac{d}{2}+1\right)_{n}} \\
& \frac{(-1)^{n+s_{2}}\left(k_{2}+k_{3}+n-l_{1}\right) !}{\left(n-k_{1}-s_{2}\right) !\left(k_{1}+k_{2}+k_{3}+s_{2}-l_{1}\right) !\left(k-k_{2}\right) ! p !\left(k_{1}-s_{1}\right) ! k_{2} !\left(k_{3}-s_{1}\right) ! s_{1} ! s_{2} !} \\
& \left(\lambda-1+l_{2}\right)_{k_{1}+k_{2}+k_{3}+s_{2}}\left(\lambda-1+l_{1}\right)_{n+k+p-k_{1}-k_{2}} \\
& \left(\frac{x^{2}}{2}\right)^{k_{1}}\left(\frac{y^{2}}{2}\right)^{n-k_{1}-s_{2}}(\zeta \cdot \eta)^{n}(x \cdot y)^{s_{2}}(\zeta \cdot x)^{k_{2}}(\zeta \cdot y)^{l_{1}-n-k_{2}}(\eta \cdot x)^{l_{2}-p-n}(\eta \cdot y)^{p}
\end{aligned}
$$

and summation over $s_{1}$ using B.11

finally gives

$$
\begin{aligned}
& \sum_{s_{1}} \frac{1}{s_{1} !\left(k_{1}-s_{1}\right) !} \frac{1}{\left(l_{2}-p-n-k_{3}+s_{1}\right) !\left(k_{3}-s_{1}\right) !} \\
& =\frac{\left(l_{2}-p-n+k_{1}\right) !}{k_{1} ! k_{3} !\left(l_{2}-p-n+k_{1}-k_{3}\right) !\left(l_{2}-p-n\right) !}
\end{aligned}
$$

$$
\begin{aligned}
& \sum \frac{l_{1} ! l_{2} !(-1)^{k+p+k_{3}}}{\left(l_{1}-k-n\right) !\left(l_{2}-p-n\right) !} \frac{1}{(\lambda)_{k+p+2 n-k_{1}+k_{3}}\left(\lambda-\frac{d}{2}+1\right)_{n}} \\
& \frac{(-1)^{n+s_{2}}\left(k_{2}+k_{3}+n-l_{1}\right) !\left(l_{2}-p-n+k_{1}\right) !}{\left(l_{2}-p-n+k_{1}-k_{3}\right) !\left(n-k_{1}-s_{2}\right) !\left(k_{1}+k_{2}+k_{3}+s_{2}-l_{1}\right) !\left(k-k_{2}\right) ! p ! k_{1} ! k_{2} ! k_{3} ! s_{2} !} \\
& \left(\lambda-1+l_{2}\right)_{k_{1}+k_{2}+k_{3}+s_{2}}\left(\lambda-1+l_{1}\right)_{n+k+p-k_{1}-k_{2}} \\
& \left(\frac{x^{2}}{2}\right)^{k_{1}}\left(\frac{y^{2}}{2}\right)^{n-k_{1}-s_{2}}(\zeta \cdot \eta)^{n}(x \cdot y)^{s_{2}}(\zeta \cdot x)^{k_{2}}(\zeta \cdot y)^{l_{1}-n-k_{2}}(\eta \cdot x)^{l_{2}-p-n}(\eta \cdot y)^{p}
\end{aligned}
$$

Now it is possible to perform summation over $k$ using B.10

$$
\begin{aligned}
& \sum_{k} \frac{(-1)^{k}}{\left(l_{1}-k-n\right) !\left(k-k_{2}\right) !} \frac{\left(\lambda-1+l_{1}\right)_{n+k+p-k_{1}-k_{2}}}{(\lambda)_{k+p+2 n-k_{1}+k_{3}}} \\
& =\frac{(-1)^{k_{2}} k_{3} !\left(\lambda-1+l_{1}\right)_{n+p-k_{1}}}{\left(l_{1}-k_{2}-n\right) !\left(n+k_{2}+k_{3}-l_{1}\right) !(\lambda)_{p+n+l_{1}+k_{3}-k_{1}}}
\end{aligned}
$$


so that we obtain

$$
\begin{aligned}
& \sum \frac{l_{1} ! l_{2} !(-1)^{k_{2}+p+k_{3}}}{\left(l_{1}-k_{2}-n\right) !\left(l_{2}-p-n\right) !} \frac{1}{(\lambda)_{p+n+l_{1}+k_{3}-k_{1}}\left(\lambda-\frac{d}{2}+1\right)_{n}} \\
& \frac{(-1)^{n+s_{2}}\left(l_{2}-p-n+k_{1}\right) !}{\left(l_{2}-p-n+k_{1}-k_{3}\right) !\left(n-k_{1}-s_{2}\right) !\left(k_{1}+k_{2}+k_{3}+s_{2}-l_{1}\right) ! p ! k_{1} ! k_{2} ! s_{2} !} \\
& \left(\lambda-1+l_{2}\right)_{k_{1}+k_{2}+k_{3}+s_{2}}\left(\lambda-1+l_{1}\right)_{n+p-k_{1}} \\
& \left(\frac{x^{2}}{2}\right)^{k_{1}}\left(\frac{y^{2}}{2}\right)^{n-k_{1}-s_{2}}(\zeta \cdot \eta)^{n}(x \cdot y)^{s_{2}}(\zeta \cdot x)^{k_{2}}(\zeta \cdot y)^{l_{1}-n-k_{2}}(\eta \cdot x)^{l_{2}-p-n}(\eta \cdot y)^{p}
\end{aligned}
$$

and arrive to the last step - summation over $k_{3}$ using B.10

$$
\begin{aligned}
& \sum_{k_{3}} \frac{(-1)^{k_{3}}}{\left(l_{2}-p-n+k_{1}-k_{3}\right) !\left(k_{1}+k_{2}+k_{3}+s_{2}-l_{1}\right) !} \frac{\left(\lambda-1+l_{2}\right)_{k_{1}+k_{2}+k_{3}+s_{2}}}{(\lambda)_{p+n+l_{1}+k_{3}-k_{1}}} \\
& =\frac{\Gamma(\lambda) \Gamma\left(\lambda-1+l_{1}+l_{2}\right)}{\Gamma\left(\lambda-1+l_{2}\right) \Gamma\left(\lambda+l_{1}+l_{2}\right)} \frac{(-1)^{k_{1}+k_{2}+s_{2}-l_{1}}}{\left(l_{1}-l_{2}-2 k_{1}-k_{2}-s_{2}+p+n\right) !\left(l_{2}-l_{1}+2 k_{1}+k_{2}+s_{2}-p-n\right) !}
\end{aligned}
$$

We see that this summation results in the key restriction $s_{2}+2 k_{1}+k_{2}+l_{2}=l_{1}+p+n$ which fixes right homogeneity properties of our polynomial as function $x$ and $y$. The sum now is over four indices and it is easy to check that after appropriate redefinition of summation variables one obtains the expression (2.33) exactly.

\section{Equivalence (2.3) and (2.54)}

When $x$ and $y$ are null vectors, the Symanzik trick allows to reduce representation (2.46) to the simpler form

$$
\begin{aligned}
& {\left[\mathbb{R}_{l_{1}, l_{2}}(u) \zeta^{\otimes l_{1}} \otimes \eta^{\otimes l_{2}}\right] \cdot\left(x^{\otimes l_{1}} \otimes y^{\otimes l_{2}}\right)} \\
& =\frac{\Gamma\left(\frac{d}{2}+l_{1}-1\right) \Gamma\left(\frac{d}{2}+l_{2}-1\right)\left(\mathrm{i} u+\frac{l_{1}+l_{2}}{2}\right) \Gamma\left(\mathrm{i} u-\frac{l_{1}+l_{2}}{2}\right)}{\Gamma\left(-\mathrm{i} u+\frac{d-2+l_{1}+l_{2}}{2}\right) \Gamma\left(\mathrm{i} u+\frac{l_{1}-l_{2}}{2}\right) \Gamma\left(\mathrm{i} u+\frac{l_{2}-l_{1}}{2}\right)} \\
& \quad \times(x-y)^{2\left(-\mathrm{i} u+\frac{d+l_{1}+l_{2}-2}{2}\right)} \int \frac{(\zeta \cdot(v-y))^{l_{1}}(\eta \cdot(v-x))^{l_{2}}}{v^{2\left(1-\mathrm{i} u-\frac{l_{1}+l_{2}}{2}\right.}(y-v)^{2\left(\frac{d}{2}+l_{1}-1\right)}(x-v)^{2\left(\frac{d}{2}+l_{2}-1\right)}} \frac{\mathrm{d}^{d} v}{\pi^{\frac{d}{2}}} .
\end{aligned}
$$

This integral is perfectly well-defined. After having stripped the right-hand side (r.h.s. ) of the $(x, y)$-independent prefactor it can be represented as (it is important to notice that 
one can impose $x^{2}=y^{2}=0$ only after having taken the derivatives):

r.h.s.

$$
\begin{aligned}
& =(x-y)^{2\left(-\mathrm{i} u+\frac{d+l_{1}+l_{2}-2}{2}\right)} \frac{\left(\zeta \cdot \nabla_{y}\right)^{l_{1}}\left(\eta \cdot \nabla_{x}\right)^{l_{2}}}{2^{l_{1}+l_{2}}\left(\frac{d}{2}-1\right)_{l_{1}}\left(\frac{d}{2}-1\right)_{l_{2}}} \int \frac{v^{2\left(\mathrm{i} u+\frac{l_{1}+l_{2}}{2}-1\right)}}{(x-v)^{2\left(\frac{d}{2}-1\right)}(y-v)^{2\left(\frac{d}{2}-1\right)}} \frac{\mathrm{d}^{d} v}{\pi^{\frac{d}{2}}} \\
& =(x-y)^{2\left(-\mathrm{i} u+\frac{d+l_{1}+l_{2}-2}{2}\right)} \frac{\Gamma\left(-\mathrm{i} u+\frac{d-2-l_{1}-l_{2}}{2}\right)\left(\zeta \cdot \nabla_{y}\right)^{l_{1}}\left(\eta \cdot \nabla_{x}\right)^{l_{2}}}{2^{l_{1}+l_{2}} \Gamma\left(\frac{d}{2}+l_{1}-1\right) \Gamma\left(\frac{d}{2}+l_{2}-1\right) \Gamma\left(1-\mathrm{i} u-\frac{l_{1}+l_{2}}{2}\right)} \\
& \int_{[0,1]^{3}} \alpha_{1}^{-\mathrm{i} u-\frac{l_{1}+l_{2}}{2}}\left(\alpha_{2} \alpha_{3}\right)^{\frac{d}{2}-2}\left[\alpha_{1} \alpha_{2} y^{2}+\alpha_{1} \alpha_{3} x^{2}+\alpha_{2} \alpha_{3}(x-y)^{2}\right]^{\mathrm{i} u+\frac{2+l_{1}+l_{2}-d}{2}} \delta\left(1-\sum_{k=1}^{3} \alpha_{k}\right) \prod_{k=1}^{3} \mathrm{~d} \alpha_{k} \\
& =\frac{\Gamma\left(-\mathrm{i} u+\frac{d-2-l_{1}-l_{2}}{2}\right)}{\Gamma\left(\frac{d}{2}+l_{1}-1\right) \Gamma\left(\frac{d}{2}+l_{2}-1\right) \Gamma\left(1-\mathrm{i} u-\frac{l_{1}+l_{2}}{2}\right)} \sum_{k} \frac{l_{1} ! l_{2} !}{k !\left(l_{1}-k\right) !\left(l_{2}-k\right) !} \frac{(x-y)^{2 k}}{(-2)^{k}} \\
& \times(\zeta \cdot \eta)^{k} \frac{\Gamma\left(\mathrm{i} u+\frac{4-d+l_{1}+l_{2}}{2}\right)}{\Gamma\left(\mathrm{i} u+\frac{4-d-l_{1}-l_{2}}{2}+k\right)} \int_{[0,1]^{3}}\left(\zeta \cdot\left(\alpha_{1} y+\alpha_{3}(y-x)\right)\right)^{l_{1}-k}\left(\eta \cdot\left(\alpha_{1} x+\alpha_{2}(x-y)\right)\right)^{l_{2}-k}
\end{aligned}
$$

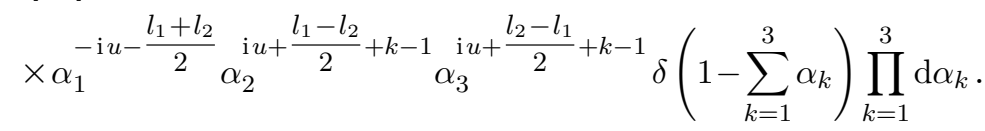

Since $\alpha_{1}+\alpha_{2}+\alpha_{3}=1$ one can write

$$
\begin{aligned}
\left(\zeta \cdot\left(\alpha_{1} y+\alpha_{3}(y-x)\right)\right)^{l_{1}-k} & =\sum_{m=0}^{l_{1}-k}\left(\begin{array}{c}
l_{1}-k \\
m
\end{array}\right)\left(\left(1-\alpha_{2}\right) \zeta \cdot y\right)^{m}\left(-\alpha_{3} \zeta \cdot x\right)^{l_{1}-k-m}, \\
\left(\eta \cdot\left(\alpha_{1} x+\alpha_{2}(x-y)\right)\right)^{l_{2}-k} & =\sum_{n=0}^{l_{2}-k}\left(\begin{array}{c}
c_{2}-k \\
n
\end{array}\right)\left(\left(1-\alpha_{3}\right) \eta \cdot x\right)^{n}\left(-\alpha_{2} \eta \cdot y\right)^{l_{2}-k-n} .
\end{aligned}
$$

The integral that then appears is of the form

$$
\begin{aligned}
& \int_{[0,1]^{3}} \alpha_{1}^{a-1} \alpha_{2}^{b-1} \alpha_{3}^{c-1}\left(1-\alpha_{2}\right)^{m}\left(1-\alpha_{3}\right)^{n} \delta\left(1-\alpha_{1}-\alpha_{2}-\alpha_{3}\right) \mathrm{d} \alpha_{1} \mathrm{~d} \alpha_{2} \mathrm{~d} \alpha_{3} \\
& =\Gamma(a) \sum_{p=0}^{m} \sum_{q=0}^{n}\left(\begin{array}{c}
m \\
p
\end{array}\right)\left(\begin{array}{c}
n \\
q
\end{array}\right)(-1)^{p+q} \frac{\Gamma(b+p) \Gamma(c+q)}{\Gamma(a+b+c+p+q)} \\
& =\Gamma(a) \Gamma(c) \sum_{p=0}^{m}\left(\begin{array}{c}
m \\
p
\end{array}\right)(-1)^{p} \frac{\Gamma(b+p)(a+b+p)_{n}}{\Gamma(a+b+c+n+p)}=\Gamma(a) \Gamma(b) \sum_{q=0}^{n}\left(\begin{array}{c}
n \\
q
\end{array}\right)(-1)^{q} \frac{\Gamma(c+q)(a+c+q)_{m}}{\Gamma(a+b+c+m+q)}
\end{aligned}
$$

where we used the Gauss identity (2.29) in the form $\sum_{l=0}^{r}\left(\begin{array}{c}r \\ l\end{array}\right)(-1)^{l} \frac{\Gamma(A+l)}{\Gamma(B+l)}=\frac{\Gamma(A)}{\Gamma(B+r)}(B-A)_{r}$. In our case the parameters actually are $a=-\mathrm{i} u+\frac{2-l_{1}-l_{2}}{2}, b=\mathrm{i} u+\frac{l_{1}+l_{2}}{2}-n$ and $c=\mathrm{i} u+\frac{l_{1}+l_{2}}{2}-m$. In particular $a+b=1-n$ so that $(a+b+p)_{n}=(1+p-n)_{n}=0$ unless $p \geqslant n$ and one of the formulas above for the integral shows that it vanishes unless $m \geqslant n$. Similarly $a+c=1-m$ so that we also need $n \geqslant m$. In the end, the integral is

$$
\begin{aligned}
& \int_{[0,1]^{3}} \alpha_{1}^{-\mathrm{i} u-\frac{l_{1}+l_{2}}{2}} \alpha_{2}^{\mathrm{i} u+\frac{l_{1}+l_{2}}{2}-n-1} \alpha_{3}^{\mathrm{i} u+\frac{l_{1}+l_{2}}{2}-m-1}\left(1-\alpha_{2}\right)^{m}\left(1-\alpha_{3}\right)^{n} \delta\left(1-\sum_{k=1}^{3} \alpha_{k}\right) \prod_{k=1}^{3} \mathrm{~d} \alpha_{k} \\
& =\delta_{m, n} n ! \frac{\Gamma\left(-\mathrm{i} u+\frac{2-l_{1}-l_{2}}{2}\right) \Gamma\left(\mathrm{i} u+\frac{l_{1}+l_{2}}{2}\right)}{\left(\mathrm{i} u+\frac{l_{1}+l_{2}}{2}\right)\left(-\mathrm{i} u+\frac{2-l_{1}-l_{2}}{2}\right)_{n}} .
\end{aligned}
$$


Putting everything together yields (we also use $(x-y)^{2}=-2 x \cdot y$ )

$$
\begin{aligned}
& \text { r.h.s. } \\
&=\frac{(-1)^{l_{1}+l_{2}} \Gamma\left(-\mathrm{i} u+\frac{d-2-l_{1}-l_{2}}{2}\right) \Gamma\left(\mathrm{i} u+\frac{l_{1}+l_{2}}{2}\right)}{\Gamma\left(\frac{d}{2}+l_{1}-1\right) \Gamma\left(\frac{d}{2}+l_{2}-1\right)\left(\mathrm{i} u+\frac{l_{1}+l_{2}}{2}\right)} \sum_{k+n \leqslant \min \left(l_{1}, l_{2}\right)} \frac{l_{1} ! l_{2} !(x \cdot y \zeta \cdot \eta)^{k}}{k ! n !\left(l_{1}-k-n\right) !\left(l_{2}-k-n\right) !} \\
& \times \frac{\Gamma\left(\mathrm{i} u+\frac{4-d+l_{1}+l_{2}}{2}\right)}{\Gamma\left(\mathrm{i} u+\frac{4-d-l_{1}-l_{2}}{2}+k\right)} \frac{(\zeta \cdot y \eta \cdot x)^{n}}{\left(-\mathrm{i} u+\frac{2-l_{1}-l_{2}}{2}\right)_{n}}(\zeta \cdot x)^{l_{1}-k-n}(\eta \cdot y)^{l_{2}-k-n} \\
&= \frac{(-1)^{l_{1}+l_{2}} \Gamma\left(-\mathrm{i} u+\frac{d-2-l_{1}-l_{2}}{2}\right) \Gamma\left(\mathrm{i} u+\frac{4-d+l_{1}+l_{2}}{2}\right) \Gamma\left(\mathrm{i} u+\frac{l_{1}-l_{2}}{2}\right) \Gamma\left(\mathrm{i} u+\frac{l_{2}-l_{1}}{2}\right)}{\Gamma\left(\frac{d}{2}+l_{1}-1\right) \Gamma\left(\frac{d}{2}+l_{2}-1\right)\left(\mathrm{i} u+\frac{l_{1}+l_{2}}{2}\right) \Gamma\left(\mathrm{i} u+\frac{4-d-l_{1}-l_{2}}{2}\right) \Gamma\left(\mathrm{i} u-\frac{l_{1}+l_{2}}{2}\right)} \\
& \times\left[\mathbb{R}_{l_{1}, l_{2}}(u) \zeta^{\otimes l_{1}} \otimes \eta^{\otimes l_{2}}\right] \cdot\left(x^{\otimes l_{1}} \otimes y^{\otimes l_{2}}\right) \\
&= \frac{\Gamma\left(-\mathrm{i} u+\frac{d-2+l_{1}+l_{2}}{2}\right) \Gamma\left(\mathrm{i} u+\frac{l_{1}-l_{2}}{2}\right) \Gamma\left(\mathrm{i} u+\frac{l_{2}-l_{1}}{2}\right)}{\Gamma\left(\frac{d}{2}+l_{1}-1\right) \Gamma\left(\frac{d}{2}+l_{2}-1\right)\left(\mathrm{i} u+\frac{l_{1}+l_{2}}{2}\right) \Gamma\left(\mathrm{i} u-\frac{l_{1}+l_{2}}{2}\right)}\left[\mathbb{R}_{l_{1}, l_{2}}(u) \zeta^{\otimes l_{1}} \otimes \eta^{\otimes l_{2}}\right] \cdot\left(x^{\otimes l_{1}} \otimes y^{\otimes l_{2}}\right) .
\end{aligned}
$$

\section{Derivative identity}

For $\zeta$ and $\eta$ two null vectors, it holds that

$$
\begin{aligned}
(\zeta \cdot \nabla)^{l_{1}}(\eta \cdot \nabla)^{l_{2}} x^{2\left(\frac{l_{1}+l_{2}+2-d}{2}+\lambda\right)}= & \frac{\left(\frac{4-l_{1}-l_{2}-d}{2}+\lambda\right)_{l_{1}+l_{2}}}{\left(\frac{4-l_{1}-l_{2}-d}{2}-\lambda\right)_{l_{1}+l_{2}}}\left(x^{2}\right)^{2 \lambda} \\
& \times\left[\mathbb{R}_{l_{1}, l_{2}}(-\mathrm{i} \lambda) \zeta^{\otimes l_{1}} \otimes \eta^{\otimes l_{2}}\right] \cdot \nabla^{\otimes\left(l_{1}+l_{2}\right)} x^{2\left(\frac{l_{1}+l_{2}+2-d}{2}-\lambda\right)} .
\end{aligned}
$$

In order to prove it one first needs to compute $y^{\otimes\left(l_{1}+l_{2}\right)} \cdot\left[\mathbb{R}_{l_{1}, l_{2}}(-\mathrm{i} \lambda) \zeta^{\otimes l_{1}} \otimes \eta^{\otimes l_{2}}\right]$ for arbitrary $y$. We use equation (2.46) to write (after having performed the integral over $z$ using the star-triangle relation)

$$
\begin{aligned}
& y^{\otimes\left(l_{1}+l_{2}\right)} \cdot\left[\mathbb{R}_{l_{1}, l_{2}}(-\mathrm{i} \lambda) \zeta^{\otimes l_{1}} \otimes \eta^{\otimes l_{2}}\right]=\frac{\Gamma\left(\frac{d}{2}-2 \lambda\right) \Gamma\left(\frac{d+l_{1}+l_{2}}{2}-1+\lambda\right) \Gamma\left(1+\frac{l_{1}+l_{2}}{2}+\lambda\right)}{\Gamma(2 \lambda) \Gamma\left(1+\frac{l_{1}+l_{2}}{2}-\lambda\right) \Gamma\left(\frac{d+l_{1}+l_{2}}{2}-1-\lambda\right)} \\
& \times y^{2\left(\frac{d+l_{1}+l_{2}}{2}-1-\lambda\right)} \int \frac{(\zeta \cdot(y-v))^{l_{1}}(\eta \cdot(y-v))^{l_{2}}}{v^{2\left(\frac{d}{2}-2 \lambda\right)}(v-y)^{2\left(\frac{d+l_{1}+l_{2}}{2}+\lambda-1\right)}} \frac{\mathrm{d}^{d} v}{\pi^{\frac{d}{2}}} \\
& =\sum_{k} \frac{l_{1} ! l_{2} !}{k !\left(l_{1}-k\right) !\left(l_{2}-k\right) !} \frac{(2 \lambda)_{k}\left(y^{2} \zeta \cdot \eta\right)^{k}(y \cdot \zeta)^{l_{1}-k}(y \cdot \eta)^{l_{2}-k}}{2^{k}\left(\lambda-\frac{l_{1}+l_{2}}{2}\right)_{k}\left(\lambda+\frac{4-l_{1}-l_{2}-d}{2}\right)_{k}} \text {. }
\end{aligned}
$$


Returning to the proof of (2.55), we can write

$$
\begin{aligned}
& {\left[\mathbb{R}_{l_{1}, l_{2}}(-\mathrm{i} \lambda) \zeta^{\otimes l_{1}} \otimes \eta^{\otimes l_{2}}\right] \cdot \nabla^{\otimes\left(l_{1}+l_{2}\right)} x^{2\left(\frac{l_{1}+l_{2}+2-d}{2}-\lambda\right)} } \\
= & \sum_{k} \frac{l_{1} ! l_{2} !}{k !\left(l_{1}-k\right) !\left(l_{2}-k\right) !}(2 \zeta \cdot \eta)^{k} \\
& \times \frac{(2 \lambda)_{k}\left(\lambda+\frac{d-2-l_{1}-l_{2}}{2}\right)_{k}}{\left(\lambda+\frac{4-l_{1}-l_{2}-d}{2}\right)_{k}}(\zeta \cdot \nabla)^{l_{1}-k}(\eta \cdot \nabla)^{l_{2}-k} x^{2\left(\frac{l_{1}+l_{2}+2-d}{2}-k-\lambda\right)} \\
= & \sum_{k, j} \frac{l_{1} ! l_{2} ! 2^{l_{1}+l_{2}-k-j}}{k ! j !\left(l_{1}-k-j\right) !\left(l_{2}-k-j\right) !}(\zeta \cdot \eta)^{k+j}(\zeta \cdot x)^{l_{1}-k-j}(\eta \cdot x)^{l_{2}-k-j} \\
& \times x^{2\left(\frac{2-l_{1}-l_{2}-d}{2}+k+j-\lambda\right)} \frac{(2 \lambda)_{k}(-1)^{k}}{\left(\lambda+\frac{4-l_{1}-l_{2}-d}{2}\right)_{k}}\left(\frac{4-l_{1}-l_{2}-d}{2}+k+j-\lambda\right)_{l_{1}+l_{2}-k-j} \\
= & \sum_{p} \frac{l_{1} ! l_{2} ! 2^{l_{1}+l_{2}-p}}{\left(l_{1}-p\right) !\left(l_{2}-p\right) !}(\zeta \cdot \eta)^{p}(\zeta \cdot x)^{l_{1}-p}(\eta \cdot x)^{l_{2}-p} x^{2\left(\frac{2-l_{1}-l_{2}-d}{2}+p-\lambda\right)} \\
& \times\left(\frac{4-l_{1}-l_{2}-d}{2}+p-\lambda\right)_{l_{1}+l_{2}-p} \sum_{k=0}^{p} \frac{1}{k !(p-k) !} \frac{(2 \lambda)_{k}(-1)^{k}}{\left(\lambda+\frac{4-l_{1}-l_{2}-d}{2}\right)_{k}} \\
= & \left(\frac{4-l_{1}-l_{2}-d}{2}-\lambda\right)_{l_{1}+l_{2}} \sum_{p} \frac{l_{1} ! l_{2} ! 2^{l_{1}+l_{2}-p}}{p !\left(l_{1}-p\right) !\left(l_{2}-p\right) !} \frac{(\zeta \cdot \eta)^{p}(\zeta \cdot x)^{l_{1}-p}(\eta \cdot x)^{l_{2}-p}}{\left(\lambda+\frac{4-l_{1}-l_{2}-d}{2}\right)_{p}} x^{2\left(\frac{2-l_{1}-l_{2}-d}{2}+p-\lambda\right)} .
\end{aligned}
$$

On the other hand, one has

$$
\begin{aligned}
(\zeta \cdot \nabla)^{l_{1}}(\eta \cdot \nabla)^{l_{2}} x^{2\left(\frac{l_{1}+l_{2}+2-d}{2}+\lambda\right)}= & \sum_{p} \frac{l_{1} ! l_{2} ! 2^{l_{1}+l_{2}-p}}{p !\left(l_{1}-p\right) !\left(l_{2}-p\right) !}\left(\frac{4-l_{1}-l_{2}-d}{2}+p+\lambda\right)_{l_{1}+l_{2}-p} \\
& \times(\zeta \cdot \eta)^{p}(\zeta \cdot x)^{l_{1}-p}(\eta \cdot x)^{l_{2}-p} x^{2\left(\frac{2-l_{1}-l_{2}-d}{2}+p+\lambda\right)} \quad \text { (D.2) }
\end{aligned}
$$

and since

$$
\left(\frac{4-l_{1}-l_{2}-d}{2}+p+\lambda\right)_{l_{1}+l_{2}-p}=\frac{\left(\frac{4-l_{1}-l_{2}-d}{2}+\lambda\right)_{l_{1}+l_{2}}}{\left(\frac{4-l_{1}-l_{2}-d}{2}+\lambda\right)_{p}}
$$

equation (2.55) does hold.

\section{E Spinor basis}

The eigenvectors of the graph-building operator (3.4) for the square-lattice fishnet have been first constructed in $d=2,4$ for any number of sites $N$ in $[12,14]$, according to the iterative formula

$$
\Psi\left(x_{1}, \ldots, x_{N}\right)=\boldsymbol{\Lambda}_{N}\left(u_{N}, l_{N}\right) \boldsymbol{\Lambda}_{N-1}\left(u_{N-1}, l_{N-1}\right) \cdots \boldsymbol{\Lambda}_{1}\left(u_{1}, l_{1}\right)
$$


where the layer operator $\boldsymbol{\Lambda}_{k}(u, n)$ acts on $k-1$ coordinates $x_{1}, \ldots, x_{k-1}$ and is defined by its integral kernel in $d=2 r$ Euclidean space

$$
\begin{aligned}
\boldsymbol{\Lambda}_{1}(u, n) & =\frac{U_{n}\left(x-x_{0}\right)}{\left(x-x_{0}\right)^{2\left(r-\frac{\tilde{\delta}}{2}-i u\right)}}, \\
\boldsymbol{\Lambda}_{k}(u, n) & =\mathbb{T}_{12}^{(n)}(i u) \mathbb{T}_{23}^{(n)}(i u) \cdots \mathbb{T}_{k-1 k}^{(n)}(i u) \frac{U_{n}\left(x_{k}-x_{0}\right)}{\left(x_{k}-x_{0}\right)^{2\left(r-\frac{\tilde{\delta}}{2}-i u\right)}},
\end{aligned}
$$

and the elementary building blocks in $d=2 r$ dimensions are

$$
\begin{aligned}
{\left[\mathbb{T}_{i j}^{(n)}(w)\right] \Phi\left(x_{i}, x_{j}\right) } & =\int d y T_{w}^{(n)}\left(x_{i}, x_{j} \mid y\right) \Phi\left(y, x_{j}\right) \\
T_{w}^{(n)}\left(x_{i}, x_{j} \mid y\right) & =\frac{U_{n}\left(x_{i}-y\right) U_{n}\left(y-x_{j}\right)^{\dagger}}{\left(x_{i}-x_{j}\right)^{2(r-\tilde{\delta})}\left(x_{i}-y\right)^{2\left(-w+\frac{\tilde{\delta}}{2}\right)}\left(y-x_{j}\right)^{2\left(w+\frac{\tilde{\delta}}{2}\right)}}
\end{aligned}
$$

The matrices $U_{n}(x)$ belong to the $n$-symmetric representation of the unitary groups $\mathrm{U}(1)$ for $2 d$ and $\mathrm{SU}(2)$ for $4 d$. For $n=1$ they defined respectively as

$$
U^{(2)}(x)=\frac{x_{1}+i x_{2}}{x_{1}-i x_{2}}=e^{i \phi}, U^{(4)}(x)=\frac{x_{\mu} \sigma^{\mu}}{\sqrt{x^{2}}}=\hat{x}_{\mu} \boldsymbol{\sigma}^{\mu},
$$

where $\boldsymbol{\sigma}_{k}=i \sigma_{k}$ for $k=1,2,3$ and $\boldsymbol{\sigma}_{4}=\mathbb{1}$, and the matrix $U_{n}(x)$ is the $n$-fold tensor products

$$
U_{n}(x)=\mathrm{U}(x) \otimes \cdots \otimes \mathrm{U}(x),
$$

namely

$$
U_{n}^{(2)}(x)=e^{i \phi n}, U_{n}^{(4)}(x)=\hat{x}_{\mu_{1}} \cdots \hat{x}_{\mu_{n}} \boldsymbol{\sigma}^{\mu_{1}} \otimes \cdots \otimes \boldsymbol{\sigma}^{\mu_{n}}
$$

The definitions E.6 can actually be extended to any even dimension $d=2 r$, for a unitary matrix $U_{n}^{(2 r)}(x)$ in the $n$-fold symmetric representation of the group $\mathrm{SU}\left(2^{r-1}\right)$

$$
U^{(2 r)}(x)=\frac{x_{\mu} \Sigma^{\mu}}{\sqrt{x^{2}}}=\hat{x}_{\mu} \Sigma^{\mu}
$$

where the matrices $\boldsymbol{\Sigma}_{\mu}$ and $\overline{\boldsymbol{\Sigma}}_{\mu}=\boldsymbol{\Sigma}_{\mu}^{\dagger}=\boldsymbol{\Sigma}_{\mu}^{-1}$ realize the Weyl spinor representation of Clifford algebra in $2 r$ dimensions

$$
\Gamma_{\mu}^{(r)}=\left(\begin{array}{cc}
0 & \boldsymbol{\Sigma}_{\mu}^{(r)} \\
\overline{\boldsymbol{\Sigma}}_{\mu}^{(r)} & 0
\end{array}\right), \quad\left\{\Gamma_{\mu}, \Gamma_{\nu}\right\}=2 \delta_{\mu \nu} \mathbb{1}_{2^{r}},
$$

that is

$$
\boldsymbol{\Sigma}_{\mu} \overline{\boldsymbol{\Sigma}}_{\nu}+\boldsymbol{\Sigma}_{\nu} \overline{\boldsymbol{\Sigma}}_{\mu}=\overline{\boldsymbol{\Sigma}}_{\mu} \boldsymbol{\Sigma}_{\nu}+\overline{\boldsymbol{\Sigma}}_{\nu} \boldsymbol{\Sigma}_{\mu}=2 \delta_{\mu \nu} \mathbb{1}_{2^{r-1}} .
$$


The concrete definition of matrices $\boldsymbol{\Sigma}_{\mu}$ and $\overline{\boldsymbol{\Sigma}}_{\mu}$ can be done recursively starting from $r=2$, according to the recipe

$$
\begin{aligned}
\Gamma_{\mu}^{(r)} & =\left(\begin{array}{cc}
0 & i \\
-i & 0
\end{array}\right) \otimes \Gamma_{\mu}^{(r-1)}, \quad \mu=1, \ldots, 2 r-2, \\
\Gamma_{2 r-1}^{(r)} & =\left(\begin{array}{cc}
0 & i \\
-i & 0
\end{array}\right) \otimes\left(\begin{array}{cc}
1 & 0 \\
0 & -1
\end{array}\right) \otimes \mathbb{1}_{2^{r-2}}, \\
\Gamma_{2 r}^{(r)} & =\left(\begin{array}{cc}
0 & 1 \\
1 & 0
\end{array}\right) \otimes \mathbb{1}_{2^{r-1}} \\
\Gamma_{k}^{(2)} & =\left(\begin{array}{cc}
0 & \boldsymbol{\sigma}_{k} \\
\overline{\boldsymbol{\sigma}}_{k} & 0
\end{array}\right), \quad \mu=1,2,3,4 .
\end{aligned}
$$

It is possible to check that with such definition $\operatorname{det}\left(x_{\mu} \boldsymbol{\Sigma}^{\mu}\right)=\operatorname{det}\left(x_{\mu} \overline{\boldsymbol{\Sigma}}^{\mu}\right)=\left(x_{\mu} x^{\mu}\right)^{2^{r-2}}$, and for a normalized vector $\hat{x}_{\mu}$ the matrices belong to the special unitary group. The definitions of layer operators in $2 d, 4 d$ provide a concrete realization of a symmetric and traceless tensor in the coordinates $x^{\mu}$ as it follows from their definition and the Fierz identity

$$
\boldsymbol{\sigma}_{\mu} \otimes \boldsymbol{\sigma}^{\mu}=2 \mathbb{1}-2 \mathbb{P}, \quad \mathbb{P}|\alpha\rangle \otimes|\beta\rangle=|\beta\rangle \otimes|\alpha\rangle .
$$

For the general $d=2 r$ situation, the same identity for the matrices $\boldsymbol{\Sigma}_{\mu}^{(r)}$ does not hold, and the layers need to be projected over specific subset of spinor components. To start with we pair each layer's $\mathrm{SU}\left(2^{r-1}\right)$ indices with generic complex vectors

$$
\left(\left\langle\left.\alpha\right|^{\otimes n}\right) \boldsymbol{\Lambda}_{k}(u, n)\left(|\beta\rangle^{\otimes n}\right)=\left(\left(\alpha^{*}\right)^{a} \boldsymbol{\Lambda}_{k}(u, n)_{a}^{b} \beta_{b}\right)^{n} \quad \text { i.e. } \quad\langle\alpha|\mathrm{U}(x)| \beta\rangle^{n} .\right.
$$

For $k=1$ the condition of symmetric traceless tensor is mapped to the null vector condition

$$
\Sigma_{\mu} \otimes \Sigma^{\mu}|\beta\rangle \otimes|\beta\rangle=\underline{0}
$$

which imposes a constraint on the components of $|\beta\rangle$. For $d=4,6$ there is no need of any such condition while for $d=2 r \geq 8$ we need to impose $N(r)$ pure spinor conditions, i.e. solve a quadratic system of $N(r)$ independent equations in the vector components. For example, $N(4)=1$ and the constraint reads

$$
\beta_{2} \beta_{5}-\beta_{1} \beta_{6}+\beta_{4} \beta_{7}-\beta_{8} \beta_{3}=0,
$$

while $N(5)=5$ and the system of constraints read

$$
\left\{\begin{array}{l}
\beta_{1} \beta_{6}-\beta_{4} \beta_{7}+\beta_{3} \beta_{8}-\beta_{5} \beta_{2}=0, \\
\beta_{6} \beta_{9}+\beta_{8} \beta_{11}-\beta_{7} \beta_{12}-\beta_{5} \beta_{10}=0, \\
\beta_{3} \beta_{12}+\beta_{6} \beta_{13}-\beta_{5} \beta_{14}-\beta_{4} \beta_{11}=0, \\
\beta_{3} \beta_{9}-\beta_{1} \beta_{11}+\beta_{7} \beta_{13}-\beta_{5} \beta_{15}=0, \\
\beta_{4} \beta_{9}-\beta_{1} \beta_{12}+\beta_{8} \beta_{13}-\beta_{16} \beta_{5}=0 .
\end{array}\right.
$$


In general the $2^{r-1}$ components of such spin vectors are subject to $N(r)>0$ linearlyindependent quadratic constraints. Imposing the latter on spin vectors, the traceless condition is valid also for the length- $k$ layer. Indeed, the matrix structure of each layer is the symmetric tensor product of an $\mathrm{SU}\left(2^{r-1}\right)$ matrix, w.r.t. which the kernel of the matrix $\boldsymbol{\Sigma}_{\mu} \otimes \boldsymbol{\Sigma}^{\mu}$ is invariant

$\boldsymbol{\Sigma}_{\mu} \otimes \boldsymbol{\Sigma}^{\mu}|\beta\rangle \otimes|\beta\rangle=\underline{0} \Longleftrightarrow(U \otimes U)\left(\boldsymbol{\Sigma}_{\mu} \otimes \boldsymbol{\Sigma}^{\mu}\right)|\beta\rangle \otimes|\beta\rangle=\underline{0} \Longleftrightarrow\left(\boldsymbol{\Sigma}_{\mu} \otimes \boldsymbol{\Sigma}^{\mu}\right)(U \otimes U)|\beta\rangle \otimes|\beta\rangle=\underline{0}$,

where $\underline{0}$ is the zero vector in the tensor product space. The proof that E. 1 is an eigenvector of the fishnet in $d=2 r$ dimensions is based on a star-triangle identity, and leads to the same eigenvalue as (4.4). Indeed, the basis E.1 and (3.14) differ only by a very non-trivial rotation in the space of tensorial indices, respect to which the spectrum is degenerate, while for $l_{j}=0$ they coincide.

\section{E.1 Star-triangle relation in $d=2 r$}

The scalar star-triangle identity in $d$-dimensions is well-known [49,62-67] and reads - in its amputated form or chain rule - as

$$
\int d^{2 r} y \frac{1}{(x-y)^{2 a}(x-y)^{2 b}}=\pi^{r} \frac{\Gamma(r-a) \Gamma(r-b) \Gamma(a+b-r)}{\Gamma(a) \Gamma(b) \Gamma(2 r-a-b)} \frac{1}{(x-y)^{2(a+b-r)}} .
$$

We can generalize it by adding an $\mathrm{SU}\left(2^{r-1}\right)$ angular part to the radial functions $x^{2}$, that is

$$
\frac{1}{x^{2 a}} \rightarrow \frac{\left\langle\alpha\left|U_{n}^{(2 r)}(x)\right| \beta\right\rangle}{x^{2 a}}=\frac{\left(\left\langle\alpha\left|\boldsymbol{\Sigma}_{\mu}^{(r)}\right| \beta\right\rangle \hat{x}^{\mu}\right)^{n}}{x^{2 a}}=\frac{\left(\left\langle\alpha\left|\boldsymbol{\Sigma}_{\mu}^{(r)}\right| \beta\right\rangle \partial^{\mu}\right)^{n}}{\Gamma\left(a+\frac{n}{2}\right) / \Gamma\left(a-\frac{n}{2}\right)(-2)^{n}} \frac{1}{x^{2\left(a-\frac{n}{2}\right)}},
$$

and obtain, for $2 r$ dimensions,

$$
\begin{aligned}
& \int d^{2 r} y \frac{\left\langle\alpha\left|U_{n}(x-y)\right| \beta\right\rangle\left\langle\alpha^{\prime}\left|U_{n^{\prime}}(y-z)\right| \beta^{\prime}\right\rangle^{*}}{(x-y)^{2 a}(y-z)^{2 b}} \\
& =\pi^{r} \frac{\Gamma\left(r-a+\frac{n}{2}\right) \Gamma\left(r-b+\frac{n^{\prime}}{2}\right) \Gamma\left(a+b-r+\frac{n^{\prime}-n}{2}\right)}{(-2)^{n} \Gamma\left(a+\frac{n}{2}\right) \Gamma\left(b+\frac{n^{\prime}}{2}\right) \Gamma\left(2 r-a-b+\frac{n+n^{\prime}}{2}\right)}\left(\left\langle\alpha\left|\Sigma_{\mu}^{(r)}\right| \beta\right\rangle \partial^{\mu}\right)^{n} \frac{\left\langle\alpha^{\prime}\left|U_{n^{\prime}}(x-z)\right| \beta^{\prime}\right\rangle^{*}}{(x-z)^{2\left(a+b-r-\frac{n}{2}\right)}} \\
& =\pi^{r} \frac{\Gamma\left(r-a+\frac{n}{2}\right) \Gamma\left(r-b+\frac{n^{\prime}}{2}\right) \Gamma\left(a+b-r+\frac{n-n^{\prime}}{2}\right)}{(-2)^{n^{\prime}} \Gamma\left(a+\frac{n}{2}\right) \Gamma\left(b+\frac{n^{\prime}}{2}\right) \Gamma\left(2 r-a-b+\frac{n+n^{\prime}}{2}\right)}\left(\left\langle\beta^{\prime}\left|\bar{\Sigma}_{\mu}^{(r)}\right| \alpha^{\prime}\right\rangle \partial^{\mu}\right)^{n^{\prime}} \frac{\left\langle\alpha\left|U_{n}(x-z)\right| \beta\right\rangle}{(x-z)^{2\left(a+b-r-\frac{n^{\prime}}{2}\right)}} .
\end{aligned}
$$

The $n$ terms or $n^{\prime}$ terms resulting from the derivation can eventually be organized in a mixing matrix for the spin vectors, and in the $r=2$ case the latter coincides with a fused $\mathrm{SU}(2)$ invariant solution of the Yang-Baxter equation. For the particular reduction $n^{\prime}=0$ (or the analogous $n=0$ ) the formula E.19 simplifies as

$$
\int d^{2 r} y \frac{\left\langle\alpha\left|U_{n}(x-y)\right| \beta\right\rangle}{(x-y)^{2 a}(y-z)^{2 b}}=\pi^{r} \frac{\Gamma\left(r-a+\frac{n}{2}\right) \Gamma(r-b) \Gamma\left(a+b-r+\frac{n}{2}\right)}{\Gamma\left(a+\frac{n}{2}\right) \Gamma(b) \Gamma\left(2 r-a-b+\frac{n}{2}\right)} \frac{\left\langle\alpha\left|U_{n}(x-z)\right| \beta\right\rangle}{(x-z)^{2(a+b-r)}} .
$$


This kind of equation is what we need in order to prove to prove that for spin vectors subject to the constraints E.13 the functions

$$
\left\langle\alpha_{N}\left|\boldsymbol{\Lambda}_{N}\left(u_{N}, l_{N}\right)\right| \beta_{N}\right\rangle \cdots\left\langle\alpha_{2}\left|\boldsymbol{\Lambda}_{2}\left(u_{2}, l_{2}\right)\right| \beta_{2}\right\rangle \cdot\left\langle\alpha_{1}\left|\boldsymbol{\Lambda}_{1}\left(u_{1}, l_{1}\right)\right| \beta_{1}\right\rangle .
$$

diagonalize the fishnet graph-building operator. The proof is identical to the $d=4$ case treated in [15], as it relies only on the star-triangle identity E.19. The main complication arising for general $r$ respect to the case $r=2$, is that the mixing of spinors is captured by a matrix that is not a solution of Yang-Baxter equation, and even contains explicitly a dependence over the coordinates. This fact can be checked already in $6 d$, when the pure spinor condition is trivial - i.e. the spin vectors components are not subject to any constraint. The main consequence is that it is not manifest the symmetry of the eigenvectors respect to the permutation of excitations numbers $\left(u_{k}, l_{k}\right)$, and for this reason we prefer to use the basis of functions (3.14) which has a much more involved structure of tensorial indices and a complicated behaviour when one or more coordinates get identified.

Open Access. This article is distributed under the terms of the Creative Commons Attribution License (CC-BY 4.0), which permits any use, distribution and reproduction in any medium, provided the original author(s) and source are credited.

\section{References}

[1] A.B. Zamolodchikov, "Fishing-net" diagrams as a completely integrable system, Phys. Lett. B 97 (1980) 63 [inSPIRE].

[2] O. Gürdoğan and V. Kazakov, New Integrable $4 D$ Quantum Field Theories from Strongly Deformed Planar $\mathcal{N}=4$ Supersymmetric Yang-Mills Theory, Phys. Rev. Lett. 117 (2016) 201602 [Addendum ibid. 117 (2016) 259903] [arXiv:1512.06704] [INSPIRE].

[3] F. Coronado, Perturbative four-point functions in planar $\mathcal{N}=4 S Y M$ from hexagonalization, JHEP 01 (2019) 056 [arXiv:1811.00467] [InSPIRE].

[4] F. Coronado, Bootstrapping the Simplest Correlator in Planar $\mathcal{N}=4$ Supersymmetric Yang-Mills Theory to All Loops, Phys. Rev. Lett. 124 (2020) 171601 [arXiv:1811.03282] [INSPIRE].

[5] J. Caetano, O. Gürdoğan and V. Kazakov, Chiral limit of $\mathcal{N}=4 S Y M$ and ABJM and integrable Feynman graphs, JHEP 03 (2018) 077 [arXiv:1612.05895] [INSPIRE].

[6] I. Prlina, M. Spradlin and S. Stanojevic, All-loop singularities of scattering amplitudes in massless planar theories, Phys. Rev. Lett. 121 (2018) 081601 [arXiv:1805.11617] [INSPIRE].

[7] B. Basso and L.J. Dixon, Gluing Ladder Feynman Diagrams into Fishnets, Phys. Rev. Lett. 119 (2017) 071601 [arXiv:1705.03545] [INSPIRE].

[8] D. Chicherin, S. Derkachov and A.P. Isaev, Conformal group: R-matrix and star-triangle relation, JHEP 04 (2013) 020 [arXiv: 1206.4150] [INSPIRE].

[9] N. Gromov, V. Kazakov, G. Korchemsky, S. Negro and G. Sizov, Integrability of Conformal Fishnet Theory, JHEP 01 (2018) 095 [arXiv: 1706. 04167] [INSPIRE].

[10] D. Chicherin, V. Kazakov, F. Loebbert, D. Müller and D.-l. Zhong, Yangian Symmetry for Fishnet Feynman Graphs, Phys. Rev. D 96 (2017) 121901 [arXiv:1708.00007] [InSPIRE]. 
[11] D. Chicherin, V. Kazakov, F. Loebbert, D. Müller and D.-l. Zhong, Yangian Symmetry for Bi-Scalar Loop Amplitudes, JHEP 05 (2018) 003 [arXiv:1704.01967] [INSPIRE].

[12] S. Derkachov, V. Kazakov and E. Olivucci, Basso-Dixon Correlators in Two-Dimensional Fishnet CFT, JHEP 04 (2019) 032 [arXiv:1811.10623] [INSPIRE].

[13] B. Basso, L.J. Dixon, D.A. Kosower, A. Krajenbrink and D.-l. Zhong, Fishnet four-point integrals: integrable representations and thermodynamic limits, JHEP 07 (2021) 168 [arXiv: 2105.10514] [INSPIRE].

[14] S. Derkachov and E. Olivucci, Exactly solvable magnet of conformal spins in four dimensions, Phys. Rev. Lett. 125 (2020) 031603 [arXiv: 1912.07588] [INSPIRE].

[15] S. Derkachov and E. Olivucci, Exactly solvable single-trace four point correlators in $\chi C F T_{4}$, JHEP 02 (2021) 146 [arXiv:2007.15049] [INSPIRE].

[16] E.K. Sklyanin, The Quantum Toda Chain, Lect. Notes Phys. 226 (1985) 196 [inSPIRE].

[17] E.K. Sklyanin, Quantum inverse scattering method. Selected topics, hep-th/9211111 [INSPIRE].

[18] E.K. Sklyanin, Separation of variables - new trends, Prog. Theor. Phys. Suppl. 118 (1995) 35 [solv-int/9504001] [INSPIRE].

[19] L.D. Faddeev, How algebraic Bethe ansatz works for integrable model, in: Quantum symmetries/Symétries quantiques. Proceedings of the Les Houches summer school, Session LXIV, Les Houches, France, August 1 - September 8 1995, Connes, A. (ed.) et al., Amsterdam North-Holland (1998) pp. 149-219. [hep-ph/9605187].

[20] L.D. Faddeev, Quantum completely integral models of field theory, Sov. Sci. Rev. C 1 (1980) 107 [INSPIRE].

[21] P.P. Kulish and E.K. Sklyanin, quantum Spectral Transform Method. Recent Development, Lect. Notes Phys. 151 (1982) 61 [InSPIRE].

[22] S.E. Derkachov, G.P. Korchemsky and A.N. Manashov, Noncompact Heisenberg spin magnets from high-energy QCD: 1. Baxter $Q$ operator and separation of variables, Nucl. Phys. B 617 (2001) 375 [hep-th/0107193] [INSPIRE].

[23] S.E. Derkachov, G.P. Korchemsky and A.N. Manashov, Separation of variables for the quantum $\mathrm{SL}(2, \mathbb{R})$ spin chain, JHEP 07 (2003) 047.

[24] A.G. Bytsko and J. Teschner, Quantization of models with non-compact quantum group symmetry: Modular XXZ magnet and lattice sinh-Gordon model, J. Phys. A 39 (2006) 12927 [hep-th/0602093] [INSPIRE].

[25] S. Kharchev, D. Lebedev and M. Semenov-Tian-Shansky, Unitary representations of $U(q)$ $($ sl $(2, R))$, the modular double, and the multiparticle $q$ deformed Toda chains, Commun. Math. Phys. 225 (2002) 573 [hep-th/0102180] [InSPIRE].

[26] S. Kharchev and D. Lebedev, Integral representation for the eigenfunctions of quantum periodic Toda chain, Lett. Math. Phys. 50 (1999) 53 [hep-th/9910265] [INSPIRE].

[27] S. Kharchev and D. Lebedev, Integral representations for the eigenfunctions of quantum open and periodic Toda chains from QISM formalism, J. Phys. A 34 (2001) 2247 [hep-th/0007040] [INSPIRE].

[28] S. Kharchev and D. Lebedev, Eigenfunctions of $G L(N, R)$ Toda chain: The Mellin-Barnes representation, JETP Lett. 71 (2000) 235 [hep-th/0004065] [INSPIRE]. 
[29] K.K. Kozlowski, Unitarity of the SoV Transform for the Toda Chain, Commun. Math. Phys. 334 (2015) 223 [arXiv: 1306.4967] [INSPIRE].

[30] N. Gromov, F. Levkovich-Maslyuk and G. Sizov, New Construction of Eigenstates and Separation of Variables for SU(N) Quantum Spin Chains, JHEP 09 (2017) 111 [arXiv: 1610.08032] [INSPIRE].

[31] J.M. Maillet and G. Niccoli, On quantum separation of variables, J. Math. Phys. 59 (2018) 091417 [arXiv: 1807.11572] [INSPIRE].

[32] P. Ryan and D. Volin, Separated variables and wave functions for rational gl(N) spin chains in the companion twist frame, J. Math. Phys. 60 (2019) 032701 [arXiv:1810.10996] [INSPIRE].

[33] J.M. Maillet and G. Niccoli, Complete spectrum of quantum integrable lattice models associated to $Y(g l(n))$ by separation of variables, SciPost Phys. 6 (2019) 071 [arXiv: 1810.11885] [INSPIRE].

[34] J.M. Maillet and G. Niccoli, On quantum separation of variables beyond fundamental representations, SciPost Phys. 10 (2021) 026 [arXiv: 1903.06618] [INSPIRE].

[35] N. Gromov, F. Levkovich-Maslyuk, P. Ryan and D. Volin, Dual Separated Variables and Scalar Products, Phys. Lett. B 806 (2020) 135494 [arXiv:1910.13442] [INSPIRE].

[36] J.M. Maillet, G. Niccoli and L. Vignoli, On Scalar Products in Higher Rank Quantum Separation of Variables, SciPost Phys. 9 (2020) 086 [arXiv: 2003.04281] [INSPIRE].

[37] N. Gromov, F. Levkovich-Maslyuk and P. Ryan, Determinant form of correlators in high rank integrable spin chains via separation of variables, JHEP 05 (2021) 169 [arXiv: 2011.08229] [INSPIRE].

[38] P. Ryan and D. Volin, Separation of Variables for Rational $\mathfrak{g l}(\mathrm{n})$ Spin Chains in Any Compact Representation, via Fusion, Embedding Morphism and Bäcklund Flow, Commun. Math. Phys. 383 (2021) 311 [arXiv:2002.12341] [INSPIRE].

[39] A. Cavaglià, N. Gromov and F. Levkovich-Maslyuk, Separation of variables and scalar products at any rank, JHEP 09 (2019) 052 [arXiv: 1907.03788] [INSPIRE].

[40] A. Cavaglià, N. Gromov and F. Levkovich-Maslyuk, Separation of variables in AdS/CFT: functional approach for the fishnet CFT, JHEP 06 (2021) 131 [arXiv:2103.15800] [INSPIRE].

[41] A.B. Zamolodchikov and A.B. Zamolodchikov, Factorized s Matrices in Two-Dimensions as the Exact Solutions of Certain Relativistic Quantum Field Models, Annals Phys. 120 (1979) 253 [INSPIRE].

[42] B. Basso, G. Ferrando, V. Kazakov and D.-1. Zhong, Thermodynamic Bethe Ansatz for Biscalar Conformal Field Theories in any Dimension, Phys. Rev. Lett. 125 (2020) 091601 [arXiv: 1911.10213] [INSPIRE].

[43] P.P. Kulish, N.Y. Reshetikhin and E.K. Sklyanin, Yang-Baxter Equation and Representation Theory. 1, Lett. Math. Phys. 5 (1981) 393 [InSPIRE].

[44] S.E. Derkachov and A.N. Manashov, Iterative construction of eigenfunctions of the monodromy matrix for SL(2, C) magnet, J. Phys. A 47 (2014) 305204 [arXiv:1401.7477] [INSPIRE]. 
[45] N.J. MacKay, New factorized S matrices associated with $\mathrm{SO}(N)$, Nucl. Phys. B 356 (1991) 729 [INSPIRE].

[46] N.Y. Reshetikhin, Hamiltonian structures for integrable field theory models. II. Models with $O(n)$ and $\mathrm{Sp}(2 k)$ symmetry on a one-dimensional lattice, Theor. Math. Phys. 63 (1985) 455 [INSPIRE].

[47] N.Y. Reshetikhin, Integrable Models of Quantum One-dimensional Magnets With $O(N)$ and Sp(2k) Symmetry, Theor. Math. Phys. 63 (1985) 555 [INSPIRE].

[48] N.J. MacKay, Rational R matrices in irreducible representations, J. Phys. A 24 (1991) 4017 [INSPIRE].

[49] K. Symanzik, On Calculations in conformal invariant field theories, Lett. Nuovo Cim. 3 (1972) 734 [INSPIRE].

[50] V.K. Dobrev et al., Harmonic Analysis on the n-Dimensional Lorentz Group and Its Application to Conformal Quantum Field Theory, Lect. Notes Phys. 63 (1977) 12059.

[51] N.I. Usyukina and A.I. Davydychev, Exact results for three and four point ladder diagrams with an arbitrary number of rungs, Phys. Lett. B 305 (1993) 136 [INSPIRE].

[52] D.J. Broadhurst, Evaluation of a Class of Feynman Diagrams for All Numbers of Loops and Dimensions, Phys. Lett. B 164 (1985) 356 [InSPIRE].

[53] A.P. Isaev, Multiloop Feynman integrals and conformal quantum mechanics, Nucl. Phys. B 662 (2003) 461 [hep-th/0303056] [INSPIRE].

[54] A.P. Isaev, Operator approach to analytical evaluation of Feynman diagrams, Phys. Atom. Nucl. 71 (2008) 914 [arXiv:0709.0419] [INSPIRE].

[55] N.I. Vilenkin, Special functions and the theory of group representations, AMS (1978).

[56] B. Basso, S. Komatsu and P. Vieira, Structure Constants and Integrable Bootstrap in Planar $N=4 S Y M$ Theory, arXiv: 1505.06745 [INSPIRE].

[57] B. Eden and A. Sfondrini, Tessellating cushions: four-point functions in $\mathcal{N}=4$ SYM, JHEP 10 (2017) 098 [arXiv:1611.05436] [INSPIRE].

[58] B. Basso, J. Caetano and T. Fleury, Hexagons and Correlators in the Fishnet Theory, JHEP 11 (2019) 172 [arXiv:1812.09794] [INSPIRE].

[59] E. Olivucci, Hexagonalization of Fishnet integrals. Part I. Mirror excitations, JHEP 11 (2021) 204 [arXiv : 2107.13035] [InSPIRE].

[60] E. Olivucci, Hexagonalization of Fishnet integrals II: form factors, work in progress.

[61] B. Eden, D.l. Plat and A. Sfondrini, Integrable bootstrap for AdS3/CFT2 correlation functions, arXiv:2102.08365 [INSPIRE].

[62] M. D'Eramo, G. Parisi and L. Peliti, Theoretical predictions for critical exponents at the $\lambda$-point of bose liquids, Lett. Nuovo Cim. 2 (1971) 878 [INSPIRE].

[63] A.N. Vasiliev, Y.M. Pismak and Y.R. Khonkonen, $1 / n$ Expansion: Calculation of the exponent $\nu$ in the order $1 / n^{3}$ by the Conformal Bootstrap Method, Theor. Math. Phys. 50 (1982) 127 [INSPIRE].

[64] A.N. Vasiliev, Y.M. Pismak and Y.R. Khonkonen, $1 / N$ Expansion: Calculation of the Exponents $\eta$ and $\nu$ in the Order $1 / N^{2}$ for Arbitrary Number of Dimensions, Theor. Math. Phys. 47 (1981) 465 [INSPIRE]. 
[65] D.I. Kazakov, Calculation of Feynman diagrams by the "Uniqueness" method, Theor. Math. Phys. 58 (1984) 223 [INSPIRE].

[66] D.I. Kazakov, The method of uniqueness, a new powerful technique for multiloop calculations, Phys. Lett. B 133 (1983) 406 [InSPIRE].

[67] A.N. Vasil'ev, The field theoretic renormalization group in critical behavior theory and stochastic dynamics, Chapman Hall/CRC (2004) [ISBN: 9780415310024]. 